\title{
Stromal disruption of pancreatic adenocarcinoma using liposomes targeted to cancer-associated fibroblasts
}

\author{
A Dissertation \\ Presented to \\ the faculty of the School of Engineering and Applied Science \\ University of Virginia \\ in partial fulfillment \\ of the requirements for the degree \\ Doctor of Philosophy \\ by \\ Lindsey Allen Taylor Brinton
}

May 2016 


\section{APPROVAL SHEET}

This dissertation is in partial fulfillment of the requirements for the degree of Doctor of Philosophy in Biomedical Engineering

Lindsey Allen Taylor Brinton

Author

This dissertation has been read and approved by the examining committee:

\section{Kimberly A. Kelly, Ph.D. Dissertation Advisor Department of Biomedical Engineering}

Kevin A. Janes, Ph.D. Committee Chair Department of Biomedical Engineering

Jason A. Papin, Ph.D. Committee Member Department of Biomedical Engineering

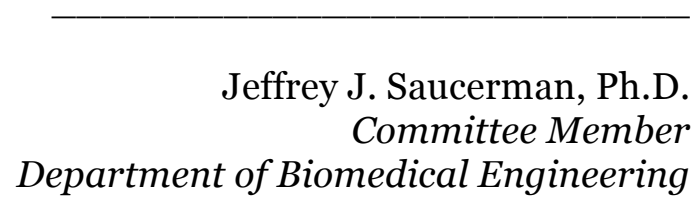

Department of Biomedical Engineering

Amy H. Bouton, Ph.D. Committee Member Department of Microbiology, Immunology, and Cancer Biology

Accepted for the School of Engineering and Applied Science:

Craig H. Benson, Ph.D. Dean, School of Engineering and Applied Science 
This thesis is dedicated to

my husband, Travis, my son, Levi, my parents, Chuck and Diane, \& my sister, Kathleen,

who have supported and helped me all along the way.

Savoir s'étonner à propos est le premier mouvement de l'esprit vers la découverte.

- Louis Pasteur

I was bold in the pursuit of knowledge, never fearing to follow truth and reason to whatever results they led.

- Thomas Jefferson 


\section{Abstract}

Over the past decade, advances in drug delivery technology have substantially increased patient compliance, improved drug efficacy, and minimized toxic side effects. With tailorable controlled release mechanisms and developments in nanotechnology, drug delivery systems can now be designed to meet specific goals. In particular, directing a drug away from healthy tissue and delivering it only to the diseased site through the use of targeted systems is paramount in battling toxicity issues. Yet obtaining the next level of clinical relevance will require significant improvements in the methods to generating targeting moieties. Similarly, molecular imaging has also rapidly progressed as a field, but is limited by a lack of quality targeting molecules. In this dissertation work, we sought to create a means of rapidly generating high affinity targeted ligands for use in therapy and imaging. We chose to apply our new approach in response to cutting edge research on the nature of pancreatic adenocarcinoma that implicates the stroma as crucial to the aggressive disease processes. The powerful combination of our new approach and the enhanced biological insight could stand to make a difference for a disease that has seen a significant lack of improvement in patient prognosis for decades.

We undertook this goal to make targeted ligands in a timely, costly, and efficient way by combining the well-established phage display technology with new quantitative analysis that we developed so that rather than relying on extensive validation and being subject to a high failure rate, we could predict which peptide sequences would be specific and selective. Our in silico quantitative analysis was made possible because of advances in 
sequencing technology that enable the reading of millions of sequences from a given library. We started by building a program to process, translate, and organize the pertinent data from deep sequencing. We then capitalized on the well-characterized results of phage screening on immobilized streptavidin protein to develop a strategy to predict specificity and selectively. We created a normalization strategy that allowed us to compare as many screens as we wanted, and we further developed our NGSanalyze program to include generation of a matrix of normalized data. We present this program as part of this dissertation and hope to enable other researchers to easily apply our process to their work.

Satisfied that our analytic methods were robust and objective, we transitioned to a more challenging task: discovering novel targeted peptides that selectively and specifically bound the cancer-associated fibroblasts (CAFs) in the stroma of pancreatic cancer. We were able to successfully implement our technique to this end and streamline the process of using such peptides to target liposomes. An impressive two of three peptide sequences chosen had great success in vivo in a mouse model. In fact, our targeted liposomes increased nanoparticle accumulation in mouse tumors $2.25^{-}$and 1.75 -fold over nontargeted nanoparticles.

We took our system one step further by loading the targeted liposomes with a CXCR2 inhibitor, which is known to act as a paracrine signal between CAFs and other tumor cells. Delivery of the inhibitor to subcutaneous pancreatic tumors using our targeted liposome displayed similar growth inhibition as intraperitoneal injection of the free drug. Additionally, immunofluorescence suggested that both the targeted liposome and free drug reduced levels of a downstream protein and decreased angiogenesis as compared to mice who received no treatment. A statistically significant decrease in cell proliferation was also detected for both the targeted liposome and free drug versus the no treatment 
group. In contrast to the free drug, however, the targeted liposomes have a very different biodistribution that enables circumvention of the toxic effects known to accompany systemic injection of the inhibitor. This proof-of-concept study shows that our targeted liposomes are capable of drug delivery and highlights their potential to improve current PDAC therapy. 


\section{Acknowledgements}

I must first and foremost thank my advisor, Kim Kelly, without whom none of this work would have been possible. You have been instrumental in my development as a scientist and in pushing me to capitalize on my strengths and improve in my weaknesses. I am a more creative, insightful, and diligent scientist because of you. It takes such an incredible commitment to take on a graduate student (we will probably all still bug you for things long after we leave) and I wish to extend my deepest gratitude for all that you have done for me.

I have spent an amazing nine years at the University of Virginia, beginning as just a young first year and reaching a time now with a family of my own and a considerable increase in knowledge. I do not think it is possible to acknowledge all the faculty, employees, and students who have helped me along this journey, but I wish to specifically thank a few. First, Jason Papin, who was my randomly assigned advisor my first year of undergraduate study and has become a valued mentor and friend. Thank you for your personal encouragement, your scientific insight, and for always challenging me to reach higher. I would like to thank the rest of my thesis committee (Kevin Janes, Jeff Saucerman, and Amy Bouton) for all of your feedback and advice. There has never been a time that I have stepped in one of your offices and not received help. I am also very grateful to my tomorrow's professor today mentor, Will Guilford. You go above and beyond for your students and I aspire to be as great of a teacher and mentor as you have 
shown me you are. Also, to my fellow tomorrow's professor today instructors and participants, especially Eric Greenwald, thank you for so many great conversations about learning, teaching, academia, and career development. I also want to acknowledge Pam Norris for her initiative in giving engineering graduate students the opportunity to teach and, on a personal note, for her role in supporting graduate students who also become mothers.

Thank you to my fellow graduate students, especially my labmates who have been there through the good and the bad. To my current labmates (Dustin Bauknight, Siva Sai Krishna Dasa, Julien Dimastromatteo, and Michael Gutknecht) what would I have done without you guys? Thank you for all of our discussions and for creating such a collaborative environment. To my former labmates (Stephanie Thomas, Marc Seaman, Soo Shin, Jaymes Beech, and Jeff Smith) thank you for showing me the ropes, supporting me in my growth as a scientist, and laughing instead of criticizing mistakes.

And last but certainly not least, I cannot but be amazed at the support and love of my family. You may not have been doing the pipetting, but in many ways, I feel like this thesis is as much yours as it is mine. To my husband, Travis, you have been so patient, caring, helpful, and encouraging. I know it's been hard to spend so much time apart, but I will always be grateful for your sacrifices on my behalf that made it possible to finish this doctorate. To my son, Levi, I know you don't realize it yet, but you've been my rock. I will never forget coming home after a long day to my little ball of energy who is so excited to discover the world around him. To my sister, Kathleen, thank you for always being there for me and listening to me about science stuff. To my "tother," Vicki, I've looked up to you ever since I can remember and am grateful for all of your enthusiasm for my research here at UVA. Finally, to my parents, Chuck and Diane, thanks for never letting me give up on my dreams and doing whatever it took to bolster me. I know you 
say you just like to visit all of the time because your grandson is growing up too fast, but it was so much more. When Levi got sick and then inevitably got me sick, you always came to take care of us (even though it meant you got sick later too). When I got busy, you lent a hand doing housework or whatever was left undone. When I had long experiments with time points at night, you came up and stayed so I could go back to the lab. I could not have done this without you.

Lindsey T. Brinton

November 2015

This work has been made possible by the generosity of the University of Virginia, the NIH pharmacological sciences training grant, and the SEAS teaching internship. 


\section{Contents}

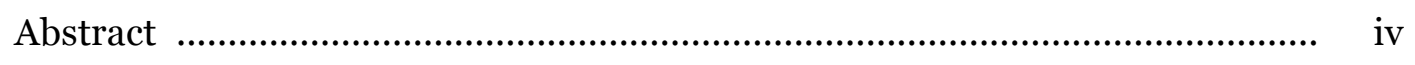

Acknowledgements .................................................................................. vii

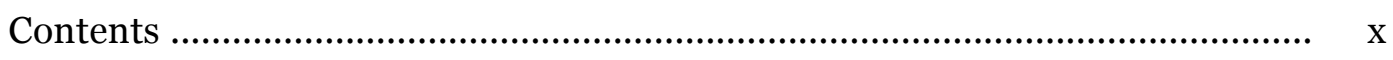

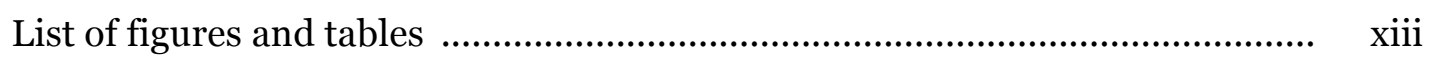

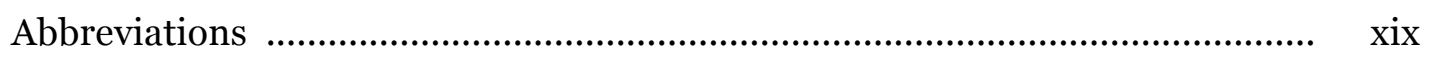

1 Dissertation aims 1

$1.1 \quad$ Introduction ...............................................................................

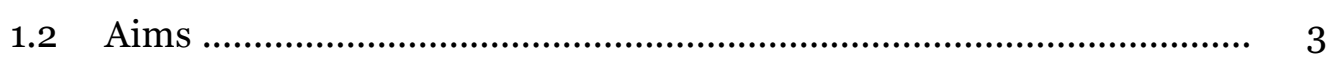

2 Background and significance $\quad 5$

2.1 Inadequate treatment options for PDAC.............................................. 6

2.1.1 Cancers of the pancreas ............................................................. 6

2.1.2 Current PDAC treatment .......................................................... 8

2.1.3 Targeting the stroma ................................................................ 10

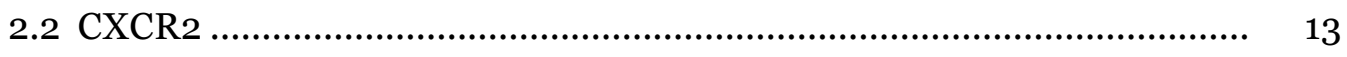

2.2.1 Physiology and biology of CXCR2 …………………................ 13

2.2.2 Inhibition of CXCR2 in PDAC ................................................. 15

2.3 Rapid and streamlined approach to developing targeted ligands and nanoparticles ........................................................................... 16

2.3.1 Targeting moieties ................................................................... 16

2.3.2 Combinatorial library screening and next generation Sequencing ........................................................................... 17

2.4 Phage as identifiers of peptide signatures ……………………........ 18 
3 New approach to targeted ligand development 22

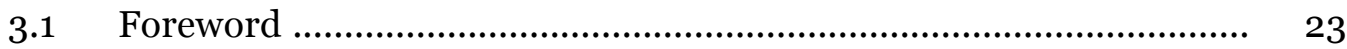

$3.2 \quad$ Introduction ….......................................................................... 23

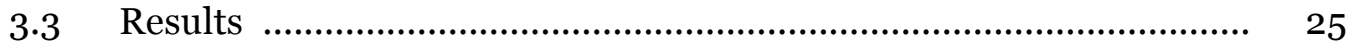

3.3.1 Process for targeted peptide sequence identification .............. 25

3.3.2 NGSanalyze .................................................................... 25

3.3.3 Purified protein screens .................................................. 28

$3.4 \quad$ Discussion ................................................................................ 29

3.5 Methods ............................................................................. 31

4 Moiety to target CAF cells $\quad 36$

4.1 Foreword …............................................................................ 37

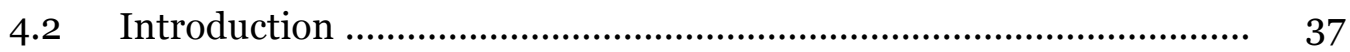

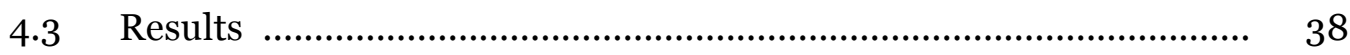

4.3.1 Targeting peptides to pancreatic CAFs ................................. 38

4.3.2 Validation of CAF-targeted peptide sequences ...................... 41

4.4 Discussion ................................................................................ 45

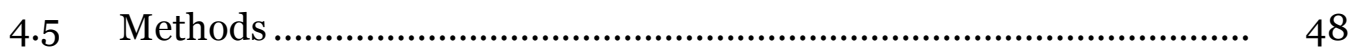

5 CAF-targeted liposomes deliver CXCR2 inhibitor 52

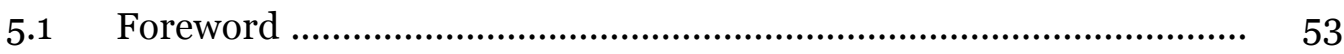

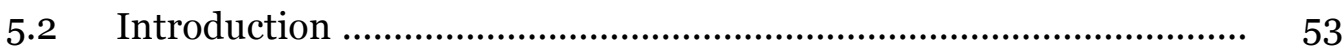

$5.3 \quad$ Results ............................................................................... 55

5.3.1 Evolution into a targeted drug delivery system ................... 55

5.3 .2 Validation of liposome targeting …...................................... 55

5.3.3 CXCR2 inhibitor decelerates tumor growth .......................... 57

5.3 .4 Blood pharmacokinetics of liposomes .................................. 61

5.3 .5 Tumor section characteristics …........................................ 62

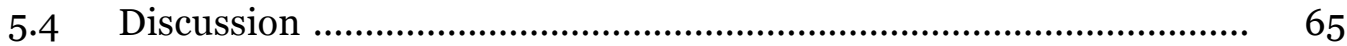

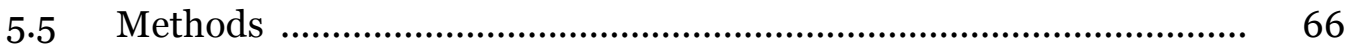

6 Dissertation discussion $\quad 70$

6.1 Developing a targeted, stromal therapy ............................................ 71

6.1.1 Historical context of our research ........................................... 71

6.1.2 Influence of an evolving biological understanding of PDAC ...... 73 
6.2 Dissertation research conclusions ................................................. 75

6.3 Future directions ........................................................................... 77

6.3.1 Imaging agent for early detection ......................................... 77

6.3.2 Binding partner identification ................................................ 78

6.3.3 Sophisticated CXCR2 studies …............................................. 79

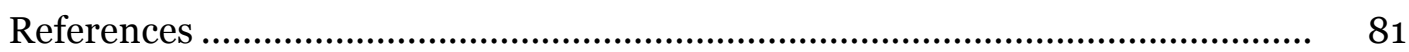

Appendix 1: NGSanalyze code .................................................................. 95

Appendix 2: Innovations in teaching nanomedicine .................................... 109

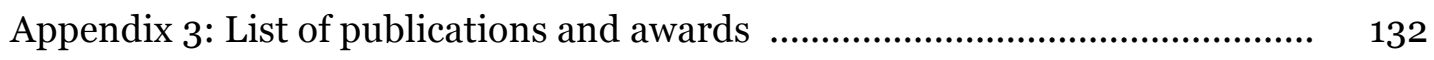




\section{List of figures and tables}

\section{Background and significance}

Figure 2.1 Pancreatic cancer statistics..........................................................

(a) Pancreatic cancer has the lowest survival rate of the common cancers, with $<7 \%$ of patients surviving five years after diagnosis. (b) Over the past few decades, the number of cases of pancreatic cancer has slightly risen; whereas, the number of deaths has remained steady. Data from [103].

Figure 2.2 Progression model for PDAC

A hypothetical model shows how a histologically normal duct can become a flat duct lesion (PanIN-1A) then a papillary duct lesion (PanIN-1B) then an atypical papillary duct lesion (PanIN-2) and finally progress to a severely atypical duct lesion/carcinoma in situ (PanIN3). Cumulative genetic mutations are indicated at the approximate stage in which they occur. Reprinted from [104].

Figure 2.3 Survival rates by stage at diagnosis

PDAC patients who present with resectable cancer have a $15-20 \%$ chance of survival; whereas, those who already have metastatic disease at time of diagnosis have a mere $2 \%$ survival rate. Data from [15].

Figure 2.4 Chemokines and chemokine receptor families

Decoy receptors (black) tend to bind multiple chemokines, while certain receptors (blue) are only known to have one ligand. Most CC (red) and CXC (green) chemokines are able to interact with multiple receptors. Therefore, inhibition of CXCR2 affects CXCL1-3 and 5-8. Reprinted from [56]. 
Figure 2.5 Peptide signatures of various tissues and cells

Peptides identified from screens performed on cell lines, ex vivo tissue specimens and in vivo screens were processed and analyzed using NGSanalyze and are presented as a heat map generated via conditional formatting in Excel.

Figure 2.6 Streamlined methodology

This overview of our methodology demonstrates our process from phage library to clinically translatable targeted drug delivery vehicle or imaging agent.

\section{New approach to targeted ligand development}

Figure 3.1 Traditional phage display process

(a) Each round of phage display consists of binding, washing, eluting, and amplifying phage. (b) An outline of the phage display process and where next generation sequencing fits in.

Figure 3.2 NGSanalyze.....

(a) Sequences obtained from the raw Illumina files. Regions flanking the displayed sequences (red) are blue and underlined. (b) A screenshot of the NGSanalyze program graphical user interface (GUI). Part 1 processes the raw data, and part 2 analyzes the data. (c) Translation of peptide sequences and calculation of read frequencies. (d) Simple libraries were created for which the appropriate scoring and ranking would be easy to calculate. (e) Predicted rankings were determined by visual inspection of each test case. For example, a peptide that is high in positive screens and very low in negative and reference screens should be predicted as an ideal peptide. Read frequencies were either absent (A), 1 (very low, VL), 10 (low, L), 100 (medium, M), or 1000 (high, H). (f) In order to allow interlibrary comparison, the data underwent two normalizations, first to read depth and then to a reference library. Data from the CAF1 screen was used to generate histograms of the entire library at each stage of the normalization process. (g) Summary of characteristics of representative screens, including the amount of time it took the NGSanalyze program to complete part 1 for each screen.

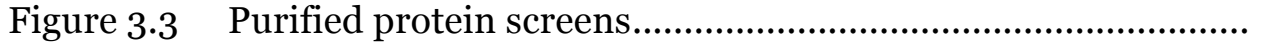

(a) The heat map represents normalized frequencies for four phage screens on streptavidin over two rounds. Screens using either glycine or biotin to elute bound 
phage were done in duplicate. Negative screens were completed on other purified protein and eluted with gycine. Sequences containing the expected HPQ motifare present at the top of the matrix based on ranking according to normalized frequency. Scatter plots across replicates of the entire screen (b), PCR (c), and sequencing $(\mathrm{d})$.

Table 3.1 Primers used for sequence indexing prior to deep sequencing

Universal primer was used with one indexing primer for each library. PCRPrimerN (FWD) was used to prepare samples for Sanger sequencing.

\section{Moiety to target CAF cells}

Figure 4.1 Process of choosing CAF-targeted peptide sequence

(a) A heat map visualization of matrix was generated using conditional formatting in Excel for the relevant cell types. The top $40 \mathrm{CAF}$-targeted and tumor epithelial cell-targeted sequences are labeled. Sequences that appear to bind all cell types are labeled as ubiquitous. Sequences selected for further validation are boxed in light blue and bolded. (b) A homology tree was generated for the CAF network. Patterns of the characteristics of the side-chains of the amino acids (positive, negative, uncharged, hydrophobic, or special case) within each sequence were used to group peptides into families. The network was written in standard Newick format and visualized using [105]. (c) In MATLAB, a random number generator was used to pull out 200 sequences from the three CAF screen repetitions and the reference library. The data was visualized as area plots with lightly shaded regions that show overlap between different screens. (d) The ratio of average round two CAF scores to round one CAF scores was calculated for the top 40 sequences. (e) Plots were generated using MATLAB showing the trajectory from round one (blue) to round two (red) of the top 40 sequences. The y-axis is the average non-targeted cell line normalized read frequency with higher frequencies indicating more binding to negative cell lines. The $\mathrm{x}$-axis is the average CAF normalized read frequency with higher frequencies indicating more binding to the CAFs.

Figure 4.2 Area plots showing overlap of 200 random sequences

between positive redundant screens and negative screens of normal cells 
(a) In MATLAB, a random number generator was used to pull out 200 sequences from the CAF screens and the normal fibroblasts. The data was visualized as area plots with lightly shaded regions that show overlap between different screen, thus darker shades of gray indicate overlap of multiple CAF screens. (b) Area plots of three sets of 200 randomly selected sequences for the CAF screens versus normal pancreatic ductal cells.

Figure 4.3 In vitro and in vivo peptide sequence validation ....................

(a) An ELISA compares the binding of phage displaying the peptides to CAFs versus normal fibroblasts (MRC5) as well as CAFs versus tumor epithelial cells (BXPC3). The first three sequences were selected using our methodology; whereas, the next six sequences were found using a traditional phage display approach. The dashed line indicates a fold change of one. (b) Flow cytometry was performed by binding fluorescentlylabeled phage to cells with a live-dead violet stain. Data was gated on cell population, live cells, and phage positive cells. (c) Phage were bound to cells, washed, and fixed. Phage amount was quantified on a plate reader by adding HRP- $\alpha$ M13 pIII antibody and TMB. Statistical significance was measured with a student ttest between HPSC and MRC5 (gray symbols) as well as between HPSC and BXPC3 (black symbols) where $\# \mathrm{p}<0.01,{ }^{*} \mathrm{p}<0.02$, and $\mathrm{p}<0.06$. (d) Fluorescentlylabeled phage were injected into mice bearing subcutaneous admix $\mathrm{CAF} / \mathrm{BXPC}_{3}$ tumors or $\mathrm{BXPC}_{3}-$ only tumors ( $\mathrm{n}=6$ tumors per group) and tumor accumulation was measured on an FMT using a regionof-interest around the tumor area. Statistical significance was determined using student's t-test of each type of displayed peptide versus KE with $\# \mathrm{p}<0.01$ and ${ }^{*} \mathrm{p}<0.02$. (e) FMT images of mice scanned 4 hours post-injection. Tumor regions have been circled with dashed lines. (f) Tumor sections were fixed, sectioned, and stained with anti- $\alpha$ SMA, then mounted with prolong gold anti-fade with DAPI. Mander's correlation coefficients (M) are indicated at the bottom of each image. Scale bars, $10 \mathrm{um}$. For each phage type, images are representative of three tumor sections of two tumors.

Figure 4.4 Epithelial content of subcutaneously injected tumors

(a) Light microscope images of $\mathrm{HE}$ stained admix CAF/BXPC3 or $\mathrm{BXPC}_{3}$ only tumor sections show stromal (s) and epithelial (e) compartments. 10x scale bar (black), 50 um. 40x scale bar (black), 10 um. 


\section{CAF-targeted liposomes deliver CXCR2 inhibitor}

Figure 5.1 Validation of peptide-conjugated liposomes

(a) A diagram showing how targeted peptides, lipophilic dye, and drug are incorporated into a liposome. (b) The NanoSight was scripted to take five videos of the liposomes. The accompanying analysis software determined the average size and concentration of liposomes in these videos. (c) Mice bearing subcutaneous admix CAF/BXPC 3 tumors $(n=10$ tumors per group) were injected with dye-labeled liposomes and tumor accumulation was measured on an FMT using a region-of-interest around the tumor area. Statistical significance was measured with a Student ttest between liposomes with and without a peptide where $\# \mathrm{p}<0.01,{ }^{*} \mathrm{p}<0.02$, and $\mathrm{p}<0.06$. (d) Tumor pharmacokinetics were determined by fitting the liposome time course data with compartment models by regression analysis of log data in MATLAB. (e) Table of tumor pharmacokinetics with AUC (area under the curve), $\mathrm{K}_{\mathrm{a}}$ (accumulation exponent), and $\mathrm{K}_{\mathrm{e}}$ (elimination exponent(s)). (f) FMT images of mice one day postinjection. (g) A biodistribution was generated by sacrificing two mice per group one day post-injection. Organs and tumors were excised and the amount of dye measured via FMT.

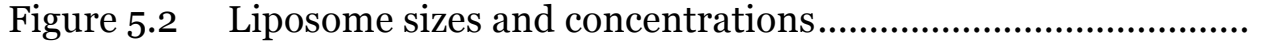

A fresh batch of liposomes was prepared weekly and analyzed via Nanosight for mean size and concentration. Because tumors on mice injected with the no peptideliposomes began to ulcerate and had to be sacrificed before the last week, only three batches of no peptideliposomes were prepared.

Box 5.1 Drug-carrying capacity of liposomes

The amount of SB225002 inside each liposome was estimated using the Nanosight analysis and the volume of liposomes per injection calculated in order to administer the same amount of SB225002 each week as with the IP injected mice.

Figure 5.3 Subcutaneous tumors

(a) Tumor growth was measured via calipers for 19 days. Dosage schedules for liposome and IP injection are shown below. (b) Representative images of HE staining of tumor sections from each treatment group show the stromal versus epithelial compartments. Scale bars, 50 um. (c) Ex vivo biodistribution was measured at the end of the study via the FMT. (d) IF images show the 
liposomes (DiR), nuclei (DAPI), and CAFs ( $\alpha \mathrm{SMA}$ ). Scale bars, 50 um.

Figure 5.4 Blood pharmacokinetics of liposomes....................................

(a) A region-of-interest (ROI) was drawn around the heart blood pool and measured as a surrogate for a blood draw. (b) The amount of dye in the heart ROI wasmeasured for 24 hours after injection via FMT. (c) A two-compartment model was used to calculate the coefficients of the first and second phases of blood clearance for both types of liposome. Artwork courtesy of Travis Brinton.

Figure 5.5 CTGF expression lowered in liposome treatment groups

(a) Proposed mechanism for increased growth due to CXCR2 inhibition. [11] (b) Western blot of homogenized tumor lysates from three mice per treatment group using anti-CTGF and anti-beta actin (loading control). (c) Densitometry of CTGF western blot.

Figure 5.6 Tumor angiogenesis

(a) Images of sections stained for CD31 were analyzed with Image J by applying the same threshold to each image and then calculating the fraction of area occupied by the vessels. (b) Representative images of CD31 staining show the vessels in different treatment groups. Scale bar, 50 um. (c) Quantification of images using six images of three sections of three tumors (54 images total per treatment group). No statistical significance found with Student's t-test for $\mathrm{p}<0.05$.

Figure 5.7 Cell proliferation

(a) Images of sections stained for Ki67 were analyzed with ImageJ (magnification of positive cell shown here) by applying the same threshold to each image and then calculating the fraction of area occupied by the stained area. Scale bar, 50 um.(b) Representative images of Ki67 staining show how the different treatment groups compare in cell proliferation. Scale bar, 50 um. (c) Quantification of images using five images of three sections of three tumors (45 images total per treatment group). \#p<0.01 statistical significance with Student's ttest.

\section{Dissertation discussion}

Figure 6.1 Evolution of cancer chemotherapy

Key historical advances in the search for effective cancer treatments. Parenthetical citations refer to references from the original article. Reprinted from [91]. 
Figure 6.2 Silver stain from pulldown assay

This silver-stained gel shows the bands that are detected from multiple pulldown assays of $\mathrm{KE}$ phage and HTTIPKV phage. Bands thought to potentially be unique are boxed.

\section{Appendix 2}

Table A2.1 The LAWA approach

First, basic understanding of each group of concepts is achieved by a quiz. Next, the understanding of these concepts is deepened through completion of an in-class worksheet. Finally, a related journal article shows how those concepts have real-world application.

Box A2.1 Sample concept-oriented multiple-choice questions

These questions test students' understanding of when a certain simplified equation can be applied and how the half-life of a drug is related to other pharmacokinetic variables.

Figure A2.1 Quantitative comparison of student performance in

traditional vs redesigned course

Students from the redesigned course were tested on three true-false questions and two short answer questions that had been used when the class was traditionally taught. The short answer questions from both types of course were evaluated with the same rubric (five metrics for the first short answer and two metrics for the second one). (a) This chart shows how the makeup of the classes differed. (b) Students from each course scored similarly on true-false questions, with the traditional type class outperforming the redesigned class on one out of three questions (* denotes statistical significance according to the chisquared test, $p=0.03$ ). (c) Students taught traditionally scored higher on the first short-answer question, while students from the redesigned course scored higher on the second short-answer question. The shape of the plotted points shows metric-to-metric variance in performance, and the area indicates overall performance on that question. All differences for the short-answer question metrics were statistically significant according to the chi-squared test. For shortanswer question one, $\mathrm{p}<0.03$ and for short-answer question two, $\mathrm{p}<0.02$. 


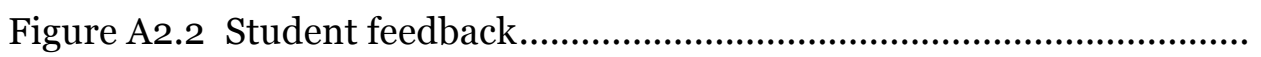

(a) Students were moderately consistent between their mid-semester and end-of-course feedback. Students felt that their learned profited most from the frequent quizzes, lectures, quiz preparation documents, and worksheets. They felt that their learning was most hindered by large and small discussions, with some students $(<20 \%)$ also feeling that the debates and worksheets were not helpful. (b) $85 \%$ of students said the worksheets increased their confidence in approaching nanomedicine-related questions. (c) $92 \%$ of students credited participation-based grading with aiding their learning.

Figure A2.3 Correlation between level of participation and grades

It appears that participating more in class did help students to receive higher grades on their exams and final projects. There was no correlation between level of participation and average quiz grades. (a) There was a weak positive correlation $(\mathrm{p}=0.06)$ between how much a student participated in class and his or her average exam grade. (b) There was a weak positive correlation $(\mathrm{p}=0.05)$ between how much a student participated in class and his or her final project grade. P-values were determined using a linear regression t-test. 


\section{Abbreviations}

$\begin{array}{ll}\text { Abbreviation } & \text { Description } \\ \text { 5-FU } & \text { 5-fluorouracil } \\ \text { ATCC } & \text { American Type Culture Collection } \\ \text { AUC } & \text { area under the curve } \\ \text { BSA } & \text { bovine serum albumin } \\ \text { BXPC3 } & \text { human pancreatic tumor epithelial cells } \\ \text { CA19.9 } & \text { PDAC serum marker used to monitor progression } \\ \text { CAF } & \text { cancer-associated fibroblast cells } \\ \text { CD31 } & \text { cluster of differentiation 31 (vessel marker) } \\ \text { CHO } & \text { Chinese hamster ovary cells } \\ \text { CT } & \text { x-ray computed tomography } \\ \text { CTGF } & \text { connective tissue growth factor } \\ \text { CXCL } & \text { chemokine with C-X-C motif } \\ \text { CXCR } & \text { receptor that binds C-X-C motif chemokines } \\ \text { DAPI } & \text { 4',6-diamidino-2-phenylindole } \\ \text { DiR } & \text { 1,1'-Dioctadecyl-3,3,3'3'-tetramethylindotricarbocyanine iodide } \\ \text { DMEM } & \text { Dulbecco's modified eagle's media for cells } \\ \text { DNA } & \text { deoxyribonucleic acid } \\ \text { DOPC } & \text { dioleoylphosphatidylcholine }\end{array}$




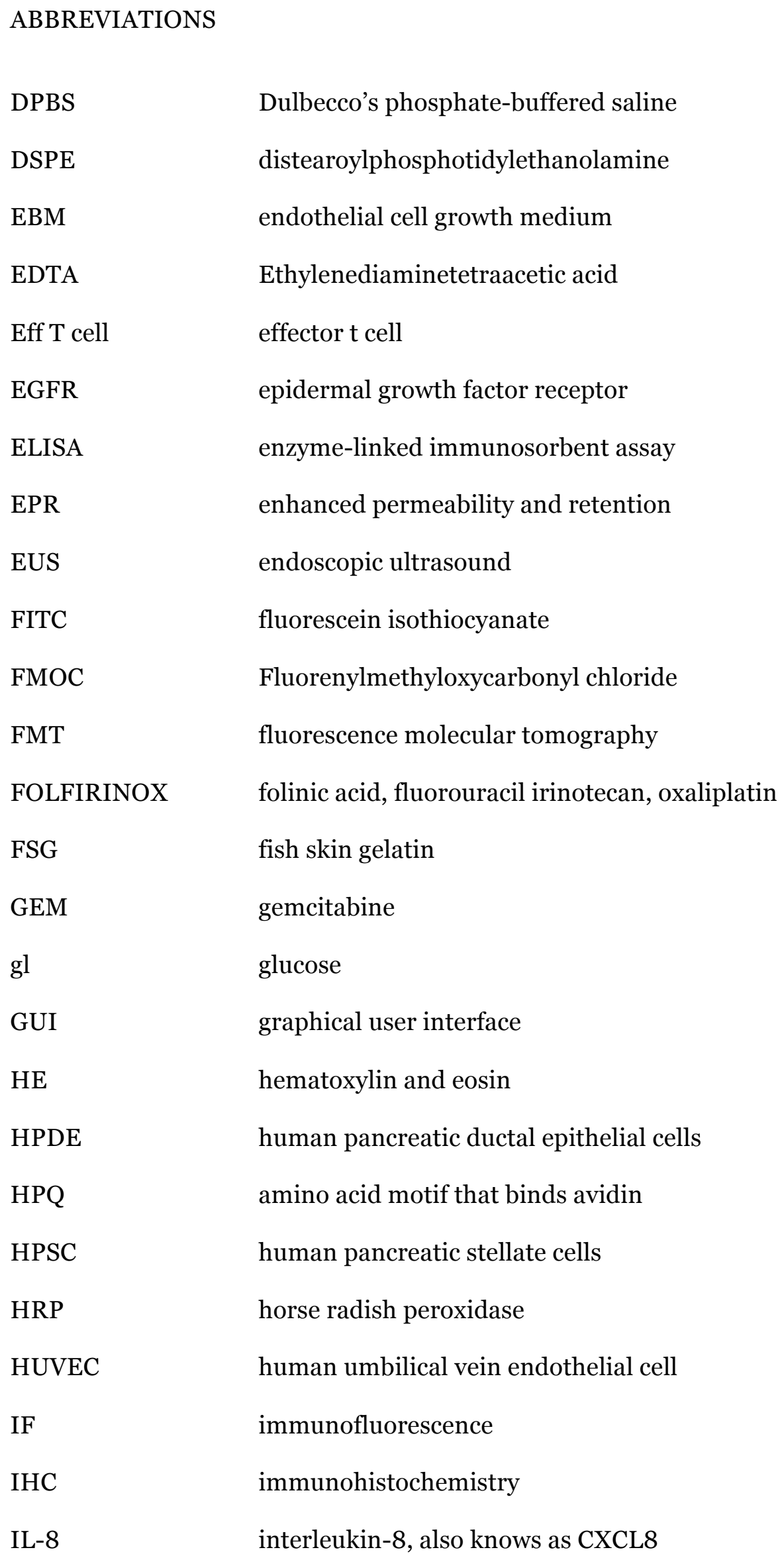




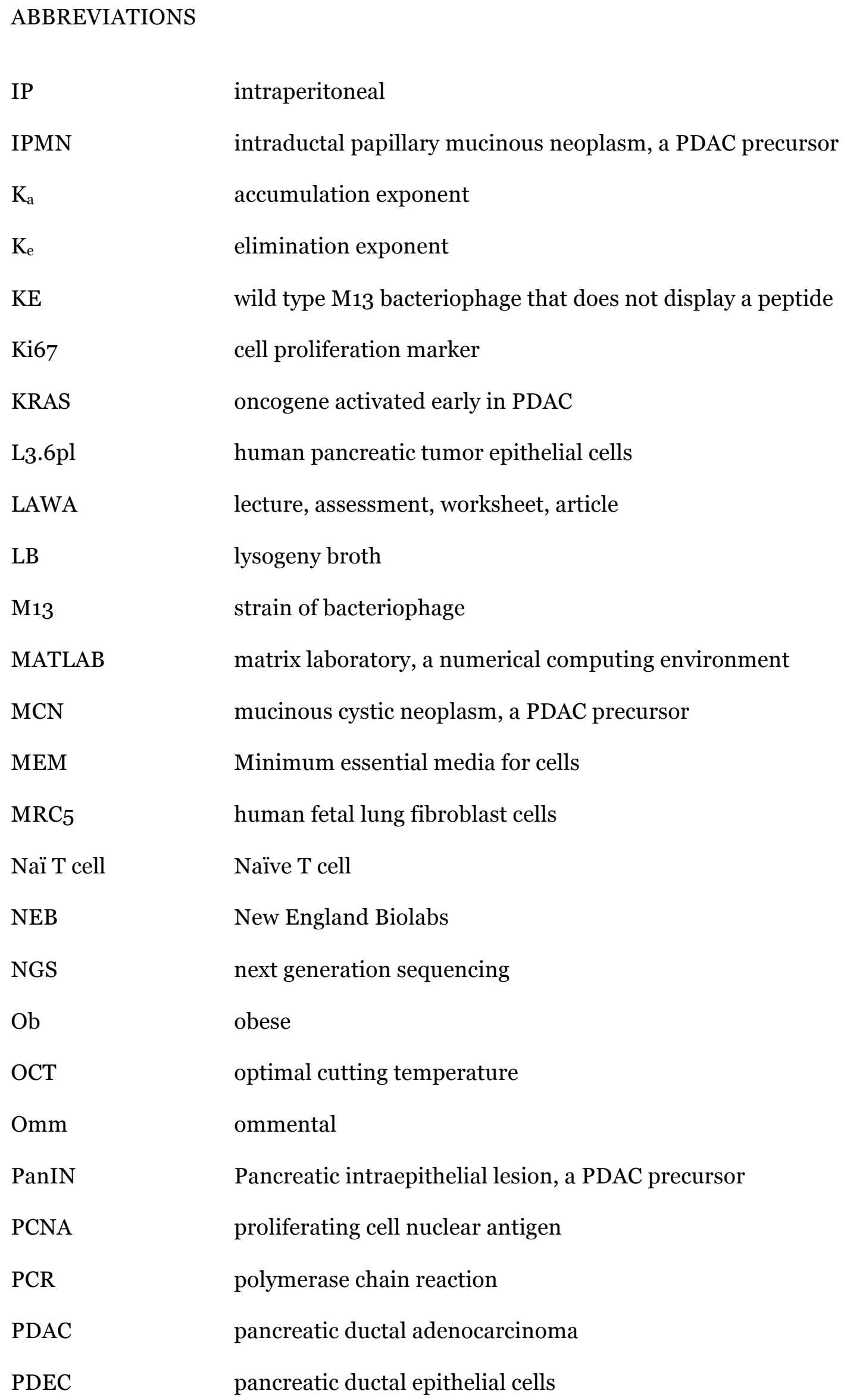




$\begin{array}{ll}\text { ABBREVIATIONS } & \\ \text { PEG } & \text { polyethylene glycol } \\ \text { PhD 7 } & \text { phage display library commercially available through NEB } \\ \text { PNET } & \text { pancreatic neuroendocrine tumor } \\ \text { RES } & \text { reticuloendothelial system } \\ \text { ROI } & \text { region-of-interest } \\ \text { RPMI } & \text { cell media developed at Roswell Park Memorial Institute } \\ \text { SILAC } & \text { stable isotope labeling by amino acids in cell culture } \\ \text { Subc } & \text { subcutaneous } \\ \text { TCM } & \text { tumor-conditioned media } \\ \text { Tet } & \text { tetracycline } \\ \text { TIL } & \text { tumor-infiltrating lymphocyte } \\ \text { TMB } & \text { tetramethylbenzidine } \\ \text { UVA } & \text { University of Virginia } \\ \text { VCAM } & \text { vascular cell adhesion protein } \\ \text { YAPC } & \text { alpha smooth muscle actin } \\ & \end{array}$


Chapter 1

Dissertation aims 


\subsection{Introduction}

Pancreatic adenocarcinoma (PDAC) is the only major cancer with 5-year survival rates in the single digits $(<7 \%)$ [1]. More than $80 \%$ of PDAC patients have progressed to latestage disease by the time they are diagnosed because pancreatic tumors evade the immune system and metastasize early, usually before symptoms start [2 3]. For these late-stage PDAC patients and an additional $15-30 \%$ of non-metastatic patients with vessel infiltration, curative surgical resection is no longer an option, so they are treated with gemcitabine [4]. Despite being the standard-of-care treatment, gemcitabine has a mere $5 \%$ partial response rate because of the rapid development of resistance and patients survive about 4 months [2 4 5]. No current treatment option provides long-term benefit for the vast majority of PDAC patients [6].

A commonality of all standard PDAC treatments is that they ignore the role of the microenvironment. Yet recent evidence indicates that PDAC acts as a complex organ system with diverse stromal cells - cancer-associated fibroblasts (CAFs), endothelial cells, and immune cells - that are interdependent and converge to create a complex network of signaling, complete with compensatory pathways. The CAFs in particular play an integral role in tumor-stromal signaling, tumor progression, immunosuppression, metastasis, and drug resistance [7]. Initially it was hypothesized that stromal depletion would improve patient prognosis through greater drug delivery and interrupted signaling. However, stromal reduction instead accelerated the cancer progression in both animal models and in a clinical trial [8 9]. Thus, we propose a more nuanced therapeutic approach that counters tumor-promoting CAF mechanisms without eliminating them to improve PDAC patient prognosis. We chose to use targeted drug delivery to avoid the toxicity or poor pharmacodynamics that have thwarted many potential PDAC drugs at the clinical level despite encouraging preclinical data [10]. Therefore, we developed a CAF targeted drug delivery system that increased local delivery to the CAFs while 
minimizing delivery to areas of toxicity such as bone marrow. To this end, we identified two CAF-selective peptides using phage display and conjugated them to liposomal nanoparticles that are biocompatible and can overcome PDAC drug delivery barriers. As a proof of concept, we delivered a drug that inhibits CXCR2, a communication hub expressed on the CAF surface that orchestrates stromal-cell-driven tumor promotion [11].

1.2 Aims

Aim 1. Development of peptide binding partners to CAFs. In order to target CAFs, we created a streamlined methodology based on phage display technology and deep sequencing that facilitated identification of CAF-selective peptides. We developed software tailored to phage display for analysis of our resultant big data sets and validated our approach using the typified HPQ-phage to streptavidin binding interaction. To ensure high specificity and selectivity, we completed both in vitro and in vivo validations. We identified two peptide sequences selective for both CAFs with favorable binding characteristics and proceeded to develop them into targeted drug delivery vehicles.

Aim 2. Generation and evaluation of CAF-targeted liposomes. To enable drug delivery to CAFs, we combined our CAF-selective ligands with our in vivo-validated targeted liposome platform. We characterized the size distribution and composition of our liposomes and studied their tumor accumulation by injecting dye-labeled liposomes in mice with subcutaneous pancreatic tumors.

\section{Aim 3. In vivo CXCR2 inhibitor delivery via CAF-targeted liposomes. CXCR2} inhibitors have mostly been studied for inflammatory diseases, but the CXCR2 ligands are also prominent in cancer signaling. Despite its promise as an important cancer therapeutic target, the safety of systemic CXCR2 inhibitors, which disrupt neutrophil 
chemotaxis and activation, is questionable for cancer patients who suffer from a compromised immune system and many of whom exhibit neutropenia as a side effect of chemotherapy [12]. Thus, we sought to use our CAF-targeted liposome to decrease the toxicity profile of CXCR2 small-molecule inhibitors. Since CXCR2 signaling does not increase PDAC ductal tumor cell proliferation in an autocrine manner, but rather affects PDAC progression through the stromal CAFs [11], the liposome should be CAF-targeted. We will loaded our CAF-targeted liposomes with a CXCR2 inhibitor and compared the tumor volume, microvessel density, IHC of the downstream signaling ligand Connective Tissue Growth Factor (CTGF), and Proliferating Cell Nuclear Antigen (PCNA) labeling observed for free CXCR2 inhibitor administration versus our targeted moiety in subcutaneous CAF-BXPC3 mouse xenograft tumors. 


\section{Chapter 2}

Background and significance 


\subsection{Inadequate treatment options for PDAC}

Despite promising advances, cancer remains the second leading cause of death in the United States, even for children ages 5-14 [13 14]. The lack of improvement in PDAC therapy efficacy in the past few decades is reflected in the essentially unchanged 5-year survival rate of $5-6 \%$ (Figure 2.1) [15]. Such poor clinical outcome is likely due to a proclivity for rapid metastasis and chemoresistance combined with the fact that most PDAC patients are not diagnosed until after the disease has metastasized [16].
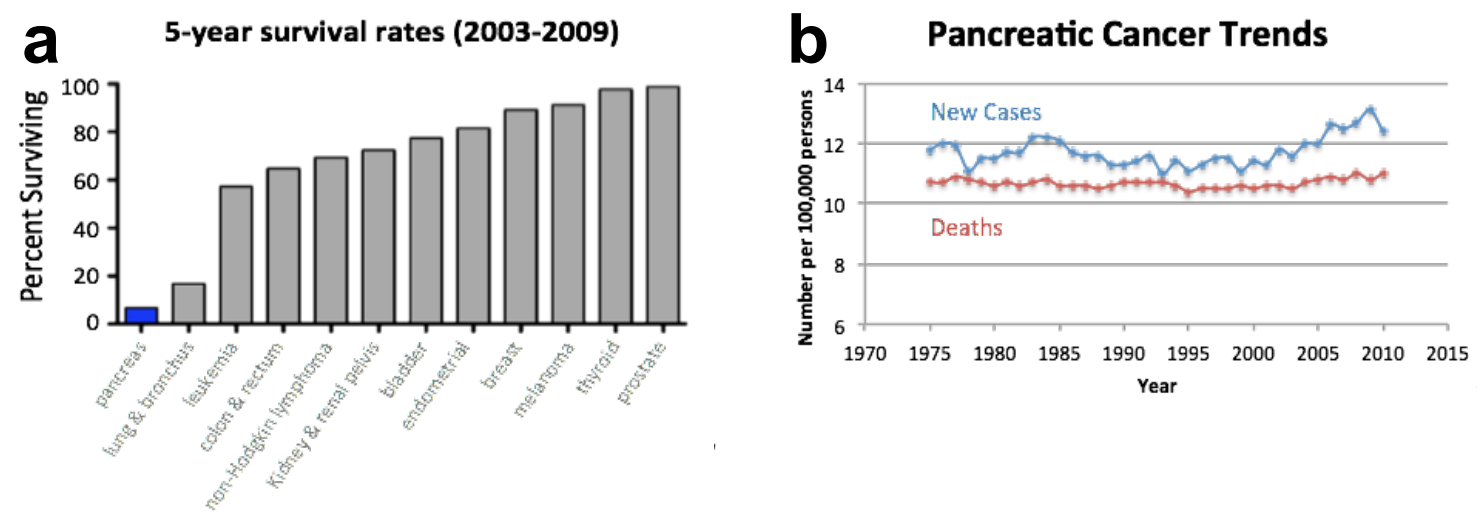

Figure 2.1: Pancreatic cancer statistics. (a) Pancreatic cancer has the lowest survival rate of the common cancers, with $<7 \%$ of patients surviving 5 years after diagnosis. (b) Over the past few decades, the number of cases of pancreatic cancer has slightly risen; whereas, the number of deaths has remained steady. Data from [103].

\subsubsection{Cancers of the pancreas}

The pancreas serves two important roles in human physiology: (1) regulation of protein and carbohydrate digestion via secretion of zymogens, and (2) maintenance of glucose homeostasis through secretion of hormones by endocrine cells. Cancer of the pancreas may develop from the endocrine cells, forming what is termed a neuroendocrine tumor (PNET); however, these tumors account for merely $5 \%$ of all pancreatic cancers. The majority of pancreatic cancers derive from the exocrine cells, typically forming in the glandular structures and therefore called adenocarcinoma (PDAC), although there are other rare types of exocrine pancreatic cancer [17]. Overall, greater than $85 \%$ of 
pancreatic cancers are classified as PDAC. The term ductal is also used in describing these adenocarcinomas, yielding the full name of pancreatic ductal adenocarcinoma due to its histological resemblance to ductal cells, not necessarily because it arises from the ductal cells. In genetic studies of mice, KRAS mutation in acinar and centroacinar cells produced PDAC precursors and PDAC; whereas neither formed with KRAS mutation in ductal cells [18]. Despite promising theories, the true "cell of origin" for pancreatic cancer remains unknown.

The three recognized precursor lesions for PDAC are pancreatic intraepithelial lesion (PanIN), intraductal papillary mucinous neoplasm (IPMN), and mucinous cystic neoplasm (MCN). PanINs are further graded from one to three according to increasing histological atypia and have been linked to a genetic progression model proposed about fifteen years ago (Figure 2.2) [19]. According to this model, genetic changes are

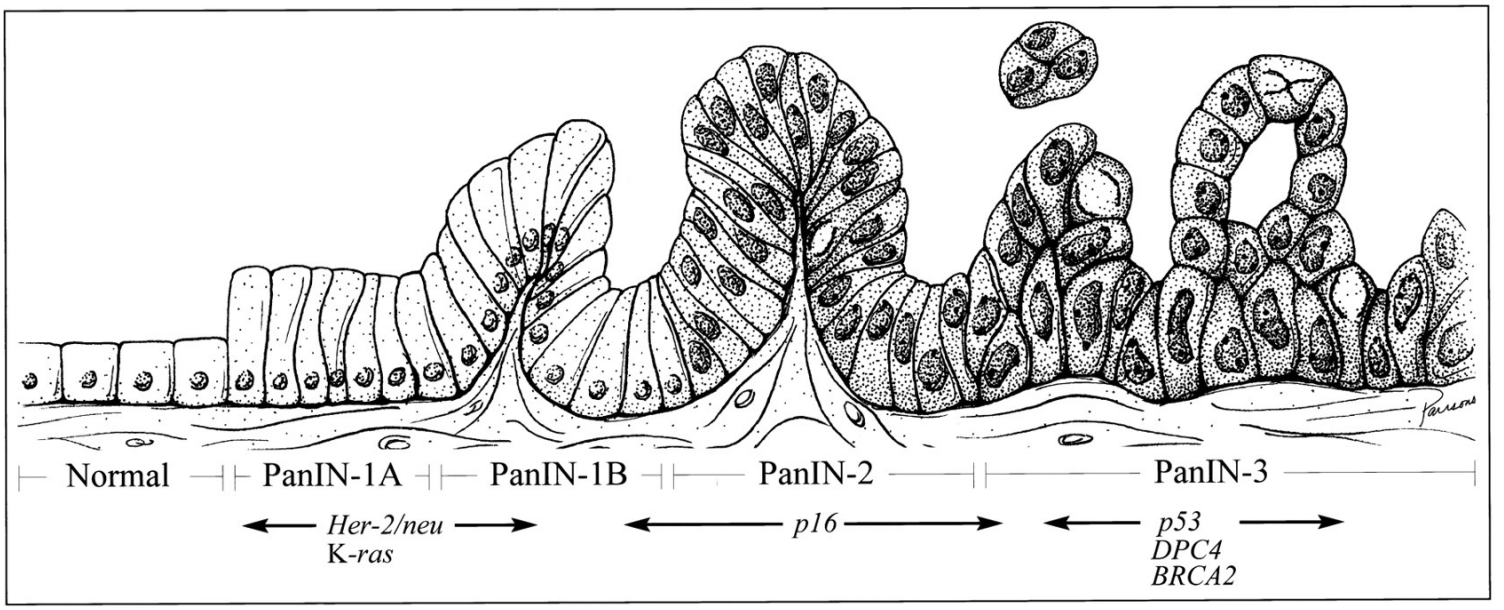

Figure 2.2: Progression model for PDAC. A hypothetical model shows how a histologically normal duct can become a flat duct lesion (PanIN-1A) then a papillary duct lesion (PanIN-1B) then an atypical papillary duct lesion (PanIN-2) and finally progress to a severely atypical duct lesion/carcinoma in situ (PanIN-3). Cumulative genetic mutations are indicated at the approximate stage in which they occur.

Reprinted from [104], "Loss of expression of Dpc4 in pancreatic intraepithelial neoplasia: evidence that DPC4 inactivation occurs late in neoplastic progression", 60/7, RE Wilentz, CA lacobuzio-Donahue, P Argani, DM McCarthy, JL Parsons, CJ Yeo, SE Kern, and RH Hruban 2002-6, copyright (2000), with permission from AACR and RH Hruban. Artwork by JL Parsons. 
accumulated starting with an activating point mutation in the Kras oncogene at the PanIN1 stage. This is followed by inactivation of the $p 16$ tumor suppressor gene around PanIN2 and the loss of $p 53, S M A D 4$, and BRCA2 tumor suppressor genes by PanIN3. This hypothesized progression model is supported by morophological analysis, clinical case studies, and molecular genetic analyses [19]. However, early PanINs will not necessarily progress to PDAC within the human lifespan as they are common in older adults and found at autopsy without PDAC. By contrast, PanIN3s are more likely to progress to PDAC and could serve a role in early detection [18]. IPMN and MCNs are less common and not as extensively studied as PanINs, but seem to present alternative routes to development of PDAC.

\subsubsection{Current PDAC treatment}

PDAC therapy fails to achieve the success of other major cancers. PDAC carries a dismal prognosis; approximately $75 \%$ of patients die just one year after diagnosis and after five years, only $5-6 \%$ of patients will still be alive [20]. Compare these statistics to five-year survival rates of other cancers like prostate (98.9\%) [21] and breast (89.4\%) [22], and the question becomes, "Why is pancreatic cancer so deadly?" PDAC is difficult to treat,

\section{Current PDAC therapy}

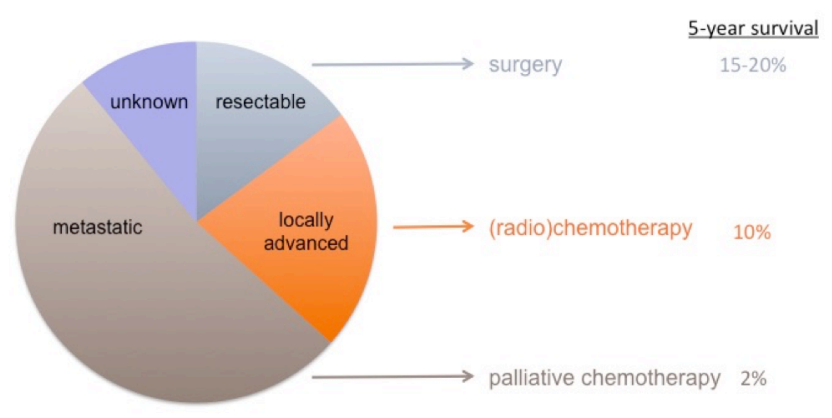

Figure 2.3: Survival rates by stage at diagnosis. PDAC patients who present with resectable cancer have a $15-20 \%$ chance of survival; whereas, those who already have metastatic disease at time of diagnosis have a mere $2 \%$ survival rate. Data from [15]. 
which is typically attributed to the disease's asymptomatic nature until late stages, causing PDAC to go undiagnosed until it is already metastatic, in addition to its aggressive characteristics and chemoresistance. In later stages, Patients can experience flu-like symptoms, cachexia, pain, and sometimes jaundice [23]. PDAC tumors are generally diagnosed and staged with computed tomography (CT) scans or sometimes with the aid of endoscopic ultrasound (EUS). Imaging determines the location, size, presence of metastases, and vascular involvement of the primary tumor in order to stratify the patient into a treatment regime. Baseline levels of tumor marker CA19.9 are also assessed to guide treatment and indicate therapeutic success.

The best-case scenario involves a resectable tumor, and these patients receive radical surgery (Whipple procedure) to remove the head of the pancreas combined with chemotherapy and sometimes radiation treatment. Yet, the majority of patients will present with unresectable disease and undergo chemotherapy or chemoradiation in order to attempt to slow the disease progression and/or palliative care such as an endoscopic stent to reduce the severe symptoms (Figure 2.3). Common chemotherapeutic agents include gemcitabine (GEM) and 5-fluorouracil (5-FU) [23]. GEM has been shown to be superior to 5 -FU, benefitting more patients $(23.8 \%$ compared to only $4.8 \%$ ) and achieving a higher overall patient survival after one year (18\% compared to $2 \%$ ) [24]. A number of clinical trials have tested the combination of GEM with other chemotherapeutics and targeted agents [25-29]. Unfortunately, most of these trials failed to improve survival and a select few reported only modest success. Two four-drug regimens (folinic acid/5-FU/irinotecan/oxaliplatin, also known as FOLFIRINOX, and GEM/cisplatin/epirubicin/5-FU) improved survival over gemcitabine alone, but also had severe associated toxicities [30 31]. Additionally, an epidermal growth factor receptor (EGFR) inhibitor, Erlotinib, in combination with 
gemcitabine was able to marginally increase survival (about two weeks) [32]. Therefore, while some limited success has been achieved, there is still a poor prognosis for the vast majority of PDAC patients.

\subsubsection{Targeting the stroma}

Current standard-of-care PDAC therapy is focused on killing ductal tumor cells, ignoring the stroma that not only makes up the vast majority of the tumor volume, but is also an integral part of the tumor progression, metastasis, and even chemoresistance [15]. The stroma is composed of immune cells, vascular cells, extracellular matrix, and CAFs that integrate to form a complex network. The CAFs, in particular, are central to the tumorpromoting aspects of the microenvironment [7 33]. For every tumor epithelial cell, there are an estimated 10-100 times more adjacent CAFs sustaining tumor growth and promoting invasion and metastasis. CAFs are ideal for targeting because they are more accessible than tumor epithelial cells and are distinctly abnormal, even at early stages, with a myofibroblast phenotype and mesenchymal markers. In addition to their facile targeting characteristics, CAFs are a pathologically sound choice of targets. They deposit extracellular matrix, stimulate tumor growth, secrete immunomodulatory signals, and promote invasion and metastasis, and thus targeted therapies are capable of disrupting each of these processes [34]. In targeting the stroma, our potential impact on PDAC is three-fold: increased biological insight, stromal therapy, and early detection imaging (Figure 2.2).

Biological insight. The role of CAFs in tumor-stromal communication is of particular interest because the pathophysiological mechanisms of such signaling and how it enables tumor progression and therapeutic resistance are not well understood in PDAC [35]. In fact, the current understanding of pancreatic carcinogenesis consists of an accumulation of genetic alterations to the tumor epithelial cells (Figure 2.2) and only recently has the 
tumor microenvironment emerged as a co-instigator of cancer. We use an unbiased screening process with a propensity to select functional targets present on the cell surface to allow for discovery of new targets on cells, which can set off a cascade of biological discovery [36-38]. We are working to optimize our previous efforts [39] to quickly identify binding partners of peptides identified by phage display by employing stable isotope labeling by amino acids in cell culture (SILAC) technology [40] with the goal of using it to identify the binding partners of the CAF-targeted peptides. More immediately, directly targeting different cell types within the tumor can augment biological understanding by inhibiting or stimulating process, lending a more systemslevel view of tumor-stromal cell interactions.

Stromal therapy. Drug delivery barriers prevent many PDAC therapies from being effective. PDAC has such a prominent desmoplastic reaction that the majority of the tumor bulk consists of stromal cells [35]. The concomitant hypovascularity is a problem even for targeted anticancer drugs that rely on tumor vessel leakiness to preferentially accumulate drug in malignant versus healthy tissue because the shortage of vessels allows more of the drug to be cleared before it enters the tumor compartment. We considered this issue in deciding both target and delivery vehicle. Our target cells, CAFs, are pro-angiogenic and preferentially located next to tumor vessels, so the drug is more likely to reach them. In fact, stromal sequestration of drug was even identified as a secondary source of decreased intracellular drug concentration in tumor epithelial cells following systemic delivery of chemotherapeutics [41]. Our delivery vehicles, liposomes, exhibit the enhanced permeability and retention (EPR) effect whereby their larger size can pass through the abnormally enlarged vessel pores, but not the smaller pores of healthy tissue. While smaller drugs are efficiently cleared by the kidneys, liposomes $>40$ $\mathrm{kDa}$ remain in circulation longer, which helps to compensate for the smaller number of 
tumor blood vessels. Liposomes are not only biocompatible, they are also optimizable; half-life, biodistribution, water solubility, and PEGylation (stability and reticuloendothelial escape) can all be modified by altering the liposomal composition [42]. Aside from drug delivery benefits, multiple peptides per liposome increases avidity and thus makes a great platform for peptides discovered via phage display where five copies of each peptide are displayed per virion during screening.

Early detection imaging. Unlike PDAC, many other cancers have seen marked improvement in survival and a group out of Taiwan assessed the source of such progress. Their analysis found that the proportion of improvement attributable to early detection versus medical technology advancement was, respectively, $77 \%$ and $23 \%$ for breast cancer, $50 \%$ and $50 \%$ for cervical cancer, $52 \%$ and $48 \%$ for colorectal cancer, $76 \%$ and $24 \%$ for gastric cancer, $66 \%$ and $34 \%$ for liver cancer, and $30 \%$ and $70 \%$ for prostate cancer [43]. Given the substantial contribution of early detection to increased survival in cancers for which the trends are improving, it stands to reason that PDAC prognosis would improve with earlier detection.

In fact, if detected early enough, curing PDAC is possible; the tumor must be surgically resected before it invades surrounding tissues for patients to have the best prognosis (Figure 2.3) [1]. Yet, this is rarely the case because the cancer tends to metastasize when it reaches about $1 \mathrm{~cm}$ in diameter, before most clinical imaging modalities are able to detect it and there are no biomarkers suitable for screening or surveillance of PDAC, even for patients who have a high risk. For diabetic adults over age 50, pancreatic cancer incidence is increased to 1 in 300 and this risk increases further with the age of the patient [44 45]. Family history of pancreatic cancer, environmental factors such as smoking, and certain genotypes can also influence risk [46-49]. Patients with $>5$-fold increased risk would likely benefit from screening imaging approaches. Combination of 
current positron emission tomography (PET) hardware that can detect lesions as small as 1-2 $\mathrm{mm}$ [50 51] with a PET agent targeted to the abundant CAF population would greatly reduce the size at which PDAC could be detected. The Kelly lab has submitted a grant to further our work in this regard and develop a PET agent by radiolabeling our CAF-targeted peptides with Gallium-68.

\subsection{CXCR2}

\subsubsection{Physiology and biology of CXCR2}

CXCR2 is a G-protein-coupled receptor known to bind interleukin-8 (IL-8), which inspired its previous name of IL-8 receptor $\beta$. It also binds several chemokine ligands, which led to its current name after the motif of these chemokine ligands that have a single amino acid between their first and second cysteine (C-X-C). CXCR2 binds several CXCLs [52 53], the most potent of which is IL-8, also known as CXCL8 (Figure 2.4). Binding of IL-8 causes neutrophil activation and inflammation [54]. In addition to neutrophils, CXCR2 is also expressed on other immune cells, such as mast cells, monocytes, and macrophages [55]. Because of this role in the inflammatory response, CXCR2 has been studied in the context of diseases in which neutrophil depletion can have beneficial effects. This includes a host of diseases associated with chronic inflammation like autoimmune diseases, cardiovascular disease, diabetes, arthritis, Alzeheimer's disease, and cancer.

Cancer-related inflammation is tied to the production of chemokines by tumor cells, CAFs, mesenchymal stem cells, endothelial cells, tumor-associated macrophages, and tumor-associated neutrophils [56]. Chronic inflammation can also be a risk factor for cancer; chronic pancreatitis increases the risk of developing PDAC sevenfold [57]. After carcinogenesis has taken place, inflammation is believed to help drive tumor progression and facilitate the processes of angiogenesis, tumor cell mobility, invasion, and metastasis 


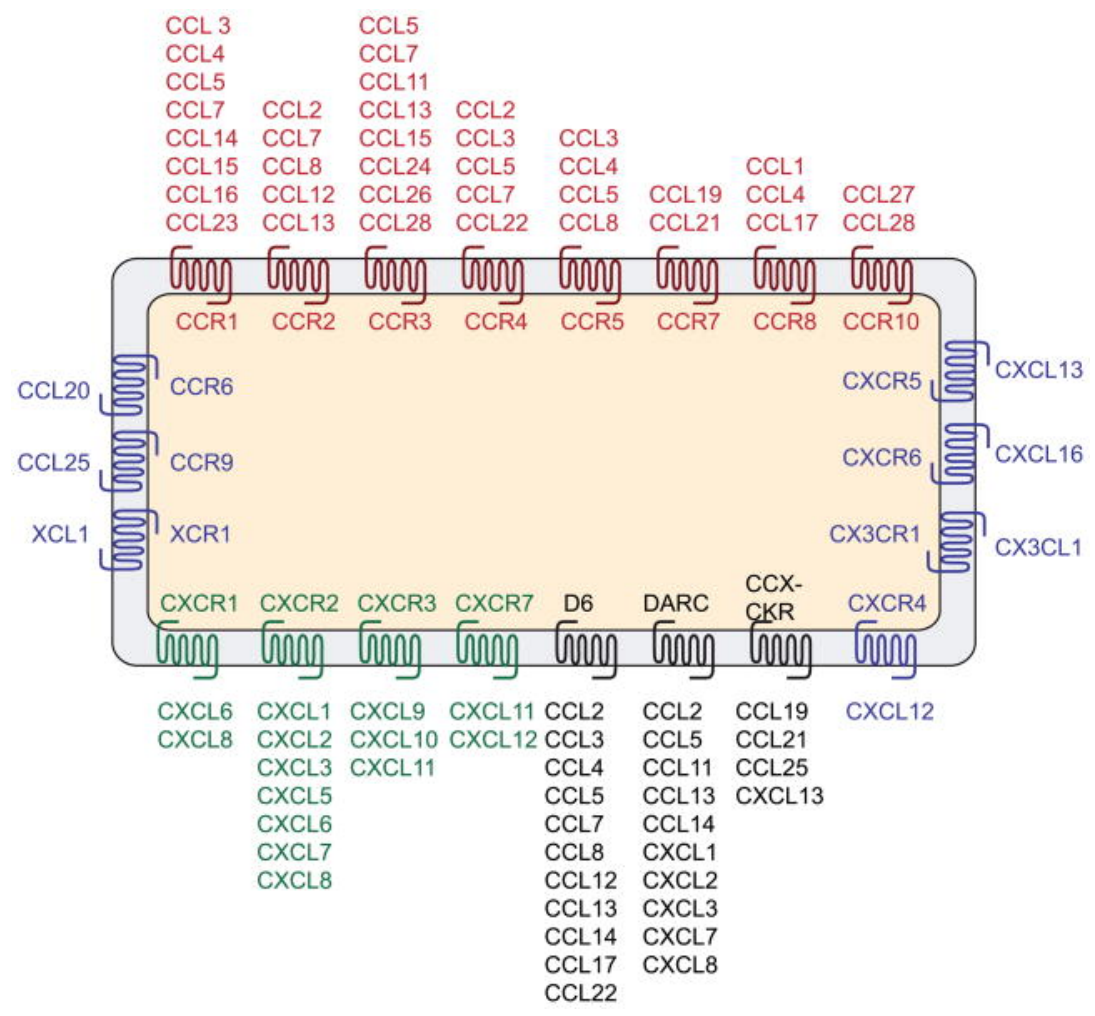

Figure 2.4: Chemokines and chemokine receptor families. Decoy receptors (black) tend to bind multiple chemokines, while certain receptors (blue) are only known to have one ligand. Most CC (red) and CXC (green) chemokines are able to interact with multiple receptors. Inhibition of CXCR2 affects CXCL1-3 and 58.

Reprinted from [56], "Chemokines and chemokine receptors: new insights into cancer-related inflammation", 16/3, G Lazennec and A Richmond, 133-144, copyright (2010), with permission from Elsevier.

[58]. Additionally, oncogenes can stimulate expression of CXCR2 on endothelial cells and promote angiogenesis [59]. Recently, it has been discovered that CXC chemokines signaling through CXCR2 cause CAFs to express Connective Tissue Growth Factor (CTGF), triggering increased fibrosis and enhancing the pro-tumor milieu [11]. Downregulation of CTGF has been shown in pancreatic and other cancers to block crosstalk between the tumor and stroma, leading to a deceleration of tumor progression [6o62]. Thus CXCR2 plays an important role in inflammation, angiogenesis, and tumor 
promotion through a variety of cells and, through CAFs in particular, is critical to tumorstroma communication that increases tumor cell proliferation.

\subsubsection{Inhibition of CXCR2 as a pilot study}

For proof of concept of stromal reprogramming using fibroblast-targeted liposomes, we wanted to select a drug that acts through CAFs, has been well characterized for systemic delivery in a PDAC mouse model, and faces clinical limitations surmountable via targeted delivery (i.e., toxicity due to neutropenia) [11 12]. CXCR2 inhibition fit these parameters with the added benefit of having available multiple formulations of smallmolecule inhibitors designed by a number of pharmaceutical companies to counter any liposomal loading difficulties that may arise. Given the multifaceted nature of the microenvironment and our systems approach, we felt that CXCR2 inhibition was a good fit because thanks to its regulatory function, CXCR2 signaling stimulates angiogenesis, causes immune cell infiltration, assists in evasion of stress-induced apoptosis, increases metastatic potential, and desensitizes cancer cells to chemotherapy [63]. The latter effect, that of inducing chemoresistance, infers that our CXCR2 inhibitor-loaded, CAFtargeted liposomes may act synergistically with current PDAC chemotherapy drugs like GEM or 5 -FU.

Broader impact. While our fibroblast-targeted liposome has the potential to transform the small-molecule CXCR2 inhibitor into a powerful PDAC drug, we expect that many other fibroblast-altering drugs could also benefit from the decreased toxicity profile for therapeutic benefit in PDAC and other epithelial tumors that also have an extensive stromal reaction, such as breast, prostate, and ovarian cancers. Apart from therapy, the fibroblast-targeted liposomes are a tool for perturbation of the complicated, integrated tumor-stromal network to increase biological understanding. Elucidation of these complex interactions should shed light on the prevailing paradox of how CAFs 
functionally contribute to both PDAC suppression and progression. Only then can researchers develop therapies capable of removing properties that foster cancer growth and progression while retaining those properties that inhibit it. Accordingly, armed with knowledge of the competing functions of stroma, researchers could break down the stromal drug delivery barriers without simultaneously creating a more invasive, undifferentiated, necrotic tumor that actually accelerates PDAC and reduces survival, as was the case with absolute CAF depletion [8].

\subsection{Rapid and streamlined approach to developing targeted ligands and} nanoparticles

\subsubsection{Targeting moieties}

Molecularly targeted affinity ligands selectively deliver drugs to diseased cells to minimize collateral toxic effects and facilitate deep, noninvasive imaging. Yet development of targeting moieties is time-consuming and expensive. Antibodies are the most commonly used high affinity ligands because they have long circulating half-lives and are not susceptible to nuclease degradation as are nucleotide-based agents. However, antibodies have disadvantages as they are produced biologically, must be humanized, and are difficult to produce on a large scale. They also vary in quality, can illicit an immune response, have limited tissue penetration, are prone to inactivation when chemically conjugated, and have a relatively short shelf life [64]. In addition, antibodies are typically developed with a particular target in mind that is selected based on years of experimentation and validation. Thus, the pace at which new affinity ligands are developed does not meet the clinical need for targeting moieties that distinguish healthy and diseased cells. 


\subsubsection{Combinatorial library screening and next generation sequencing}

High throughput screening of combinatorial chemical libraries is a powerful approach for identifying targeted molecules. The display of combinatorial peptide libraries on the surface of bacteriophage provides an efficient and rapid way to search billions of peptides for specific binding properties. Phage display offers a number of important advantages such as rapid and economical biological expansion, vast peptide diversity, a rapid screening process, and the availability of many types of phage clones and libraries (for review see [65]). Another important advantage is that bacteriophage, unlike higher organisms, have only one copy of each gene, so it is easy to identify the displayed peptide of a clone by sequencing the appropriate portion of the phage genome. Further, peptide sequences are easily manufactured, non-immunogenic, stable, and easily modified, making them ideal for clinical translation [36-38]. However, current screening protocols are hampered by false positive rates caused by non-specific phage binding and unequal rates of amplification [66] as well as by loss of potential candidates early in the process due to low starting phage concentrations. Furthermore, it is necessary to hand pick and Sanger sequence each individual plaque at the end of the traditional screening process, a labor intensive and costly process that decreases the data output to about one ten thousandth of the final enriched phage pool. Therefore, we sought to harness the power of phage display screening and take advantage of advances in sequencing to develop a method that allows discovery of molecular signatures of peptides that can selectively distinguish cells in vitro and in vivo.

Next generation sequencing (NGS) provides a potential avenue to overcome some of the drawbacks associated with phage screening by greatly increasing the depth of characterization of post-screening libraries [66-68]. Rather than hand-picking plaques and Sanger sequencing them at the conclusion of biopanning, a small aliquot of the DNA 
from the final phage yield is indexed and sequenced on an Illumina platform system. In phage display approaches, NGS can introduce a form of quantification, and increase the yield of sequences from hundreds to millions, reaching beyond the so-called parasitic phage that tend to dominate traditional screens because they amplify more rapidly [66]. The challenge in using NGS phage screens for rapid targeted ligand production is determining which sequences represent high-specificity ligands as the results conflate enrichment due to ligand specificity with the enrichment due to non-specific binding and variation in amplification rate. The inability to easily separate these groups necessitates extensive validation and dramatically decreases the efficiency of targeted ligand discovery. An additional hurdle to use and clinical translation exists in eliminating highspecificity ligands that lack selectivity because they bind not only to the cell of interest but also other cells under systemic administration. Thus we developed a comprehensive in silico analysis that can predict selective peptide sequences and circumvent many of these weaknesses of phage display.

\subsection{Phage as identifiers of peptide signatures}

As an extension of our development of quantitative in silico analysis across multiple libraries, a more comprehensive view of phage-peptide interactions with tissues and cells began to emerge. In compiling data from 56 different screens performed in vitro on cells, in vivo in mice, or ex vivo on tissue, we found that groups of phage were formed reminiscent of molecular signatures associated with microarray analysis (Figure 2.5). We hypothesize that using our analysis, peptide signatures could be generated for different tumor types that would reflect the unique environment on the cell surface, and plan to pursue this line of research as we gather more data.

In this dissertation, we present our methodology that rapidly generates highly specific and selective ligands with facile nanoparticle incorporation to create translatable, 
targeted drug delivery or imaging systems (Figure 2.6). Our approach can be universally applied from simple purified protein screens to more complex in vitro cell, in vivo, or ex vivo tissue screening. Compared to the traditional phage display screening methodology, we believe that our methodology results in rapid identification of selective peptides with favorable characteristics without the necessity of first identifying the target of interest. We demonstrate that our rapid method for identifying affinity ligands has great utility for detecting and delivering payloads to the cells of interest by employing them to target CAFs in PDAC. Our CAF-targeted liposomes present a novel means of tackling this particularly devastating disease and show potential in a proof-of-concept study where they deliver a CXCR2 inhibitor to slow tumor growth. 


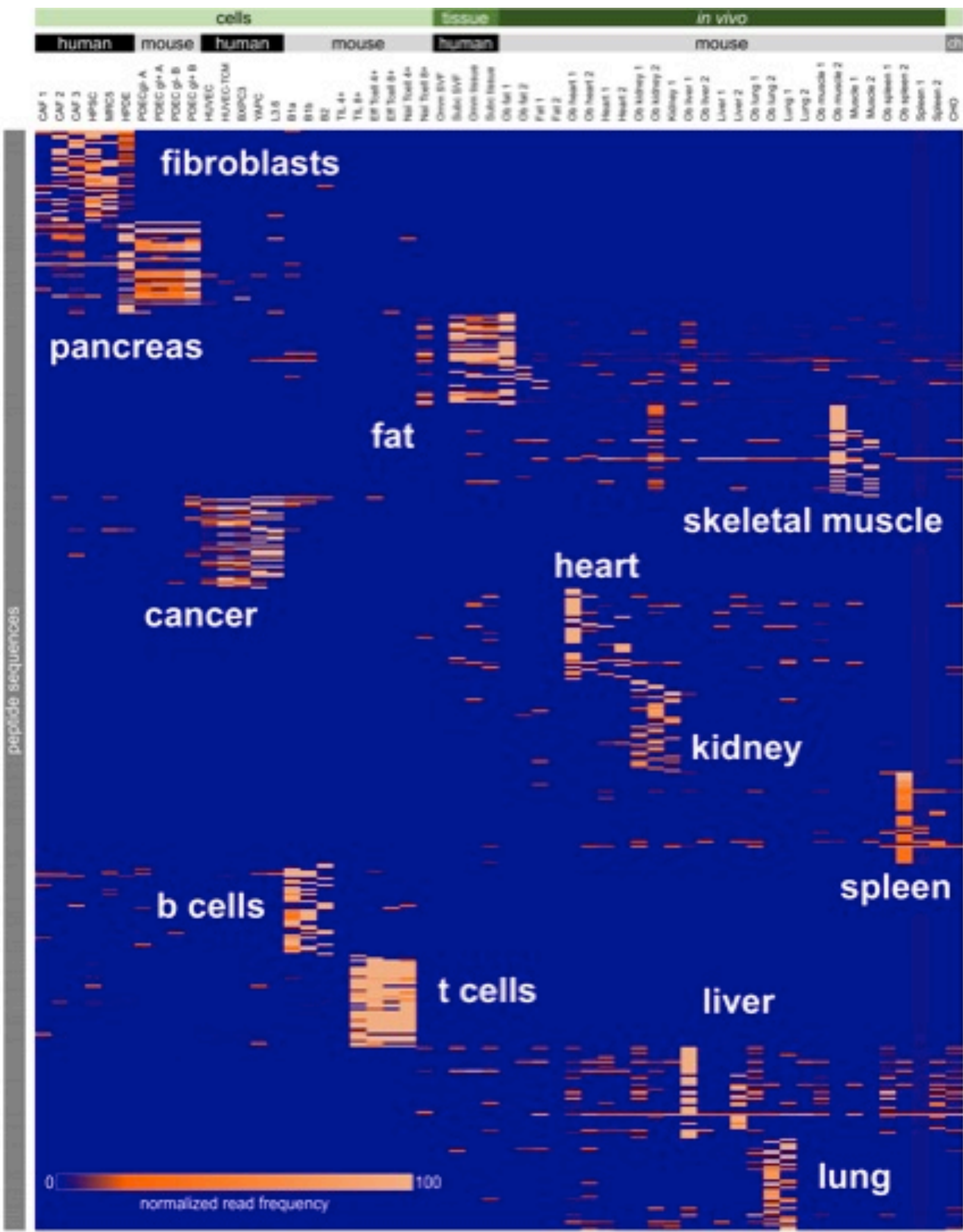

Figure 2.5: Peptide signatures of various tissues and cells. Peptides identified from screens performed on cell lines, ex vivo tissue specimens and in vivo screens were processed and analyzed using NGSanalyze. The data is presented as a heat map generated via conditional formatting in Excel. 


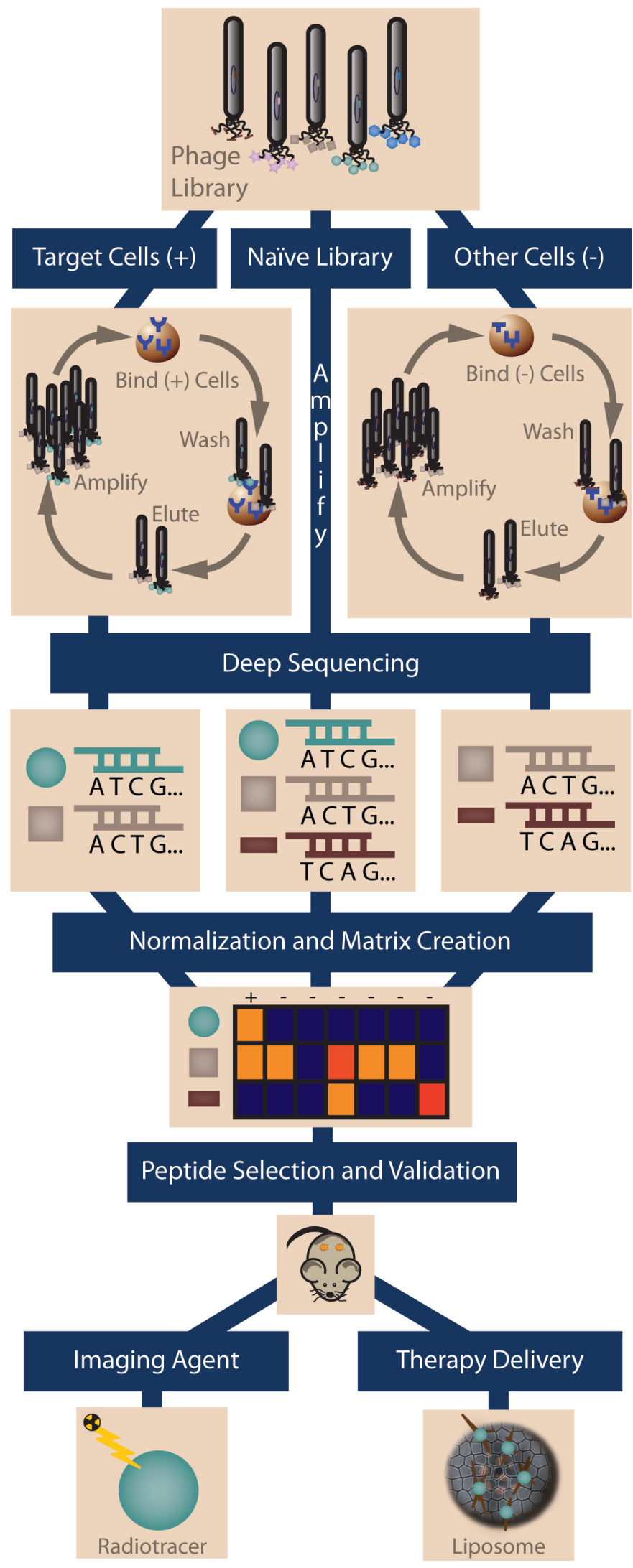

Figure 2.6: Streamlined methodology. This overview of our methodology demonstrates our process from phage library to clinically translatable targeted drug delivery vehicle or imaging agent. 
Chapter 3

New approach to

targeted

nanoparticle

development 


\subsection{Foreword}

We were able to improve analytic predictability within the framework of phage display technology via in silico mimicry of in vivo conditions, an idea which stemmed from two observations during our work using the traditional phage display method to identify various targeting peptides. The first observation was that subtractive or depletion techniques intended to prevent targeting peptides from binding more than one cell type are time consuming and hampered by the presence of large numbers of parasitic, rapidly amplifying phage. Such subtractive techniques can only be used to remove phage selective for one, perhaps two, cell lines; yet, physiologically, more cell types exist and should be included in the analysis. Furthermore, specificity of the selected peptides is hindered by insufficient subtraction; a nonspecific clone can be amplified and perpetuated in the rest of the screening process. Conversely, a specific clone is lost if it is removed during subtraction due to non-specific binding to the negative cell. The second observation is that the inefficiencies of the process, especially the lengthy validations, limit the timetable of translatability to the clinic. Our observations inspired creation of a streamlined technique that harnesses the power of quantification and big data processing in order to ensure selectivity, enhance specificity, and reduce time-consuming validation steps to rapidly generate a clinically relevant, targeted nanoparticle.

\subsection{Introduction}

Phage display screens use a high-diversity library to rapidly and cheaply identify a binding compound. Ideally, multiple selective rounds increase selective pressure to ensure identification of a peptide with high affinity and high specificity. In reality, many factors obfuscate the results such that extensive validation is required. While some sources of error or bias like low initial concentrations of phage are easily adjusted within the current framework of traditional phage display screening, other factors that skew 
results require new methods. Typically, non-specific binding, amplification bias, and the final sampling of just a tiny portion of the remaining pool of binding phage culminate in a number of "false positives" [69 70]. In response to these methodological disadvantages, deep sequencing of the final phage pool was introduced as a means of quantifying the results and expanding the depth of sequencing (Figure 3.1). Now with millions of sequences, the small final sampling was increased and amplification bias was reduced by cutting out many of the amplification steps [67 71]. We further developed use of next generation sequencing with phage by creating a MATLAB program to translate and analyze the millions of results produced by each screen. We also developed a unique analysis technique that increases predictability of highly selective and specific phage and generated a new system of grouping resultant phage into families.

a.

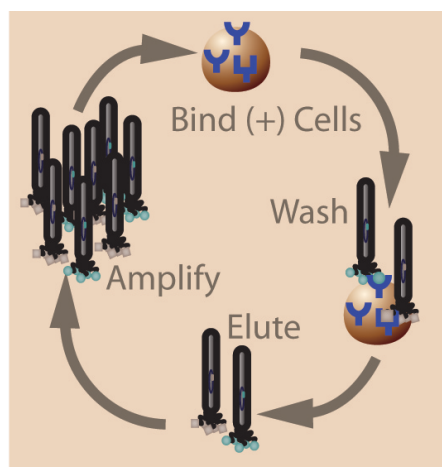

b.

Traditional phage display steps: Next-generation deep sequencing replaces steps 5 and 6 after repeating steps 1-4 twice

1. Expose phage to target

2. Wash away unbound phage

3. Elute bound phage (ex. pH change)

4. Amplify isolated phage

${ }^{*}$ Repeat 1-4 until find selective phage *

5. Titer phage and pick phage plaques

6 . Sanger sequence picked phage

7. Validate picked phage

Figure 3.1: Traditional phage display process. (a) Each round of phage display consists of binding, washing, eluting, and amplifying phage. (b) An outline of the phage display process and where next generation sequencing fits in. 


\subsection{Results}

\subsubsection{Process for targeted peptide sequence identification}

Using commercially available phage display libraries, we performed in vitro or in vivo selection followed by in silico analysis to identify peptides selective for each cell or tissue type (Figure 2.5). In total, we have screened 56 cells or tissues by performing two rounds of biopanning with a PhD 7 amino acid phage library on indicated cells or in vivo conditions and deep sequencing the DNA isolated post-screening. The resultant NGS data $(84,232,601$ reads) was normalized to remove non-specificity selection bias, then compiled into a quantitative matrix that facilitates multi-screen comparisons and identifies peptides that are both specific and selective for the assessed cell or tissue types. Consequently, our method shifts the burden of analysis away from subjective examination that requires a lot of expertise and lends a degree of flexibility to the screening process as additional cell lines can be added later and compared to previous screens.

\subsubsection{NGSanalyze}

The handling of the large datasets from quantitative sequencing, normalization and matrix generation necessitated creation of a user-friendly MATLAB-based software that automates the analysis process (Figure 3.2). Part 1 of our software extracts the variable regions from the reads generated by deep sequencing the biopanning results, translates the sequences, and then determines the frequency of appearance of each displayed peptide (Figure 3.2 a-c; see Appendix 1 for code). It also (optionally) removes any reads with codons that do not correspond to the reduced genetic code used by NEB to generate the $\mathrm{PhD} 7$ library, thus decreasing the number of misreads. The software is easy to manipulate in order to adapt it to any phage display library either commercially available 
a.

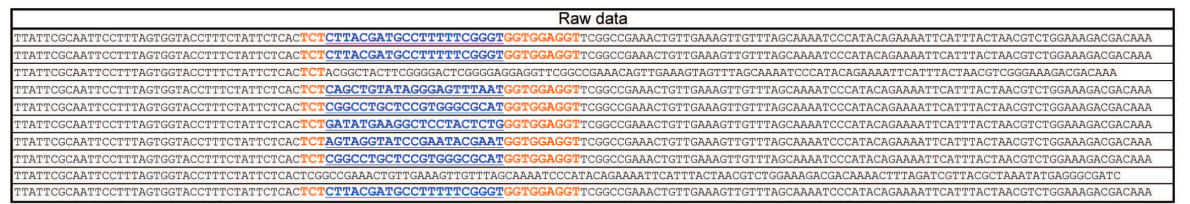

b.

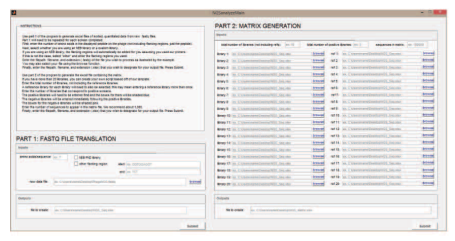

C.

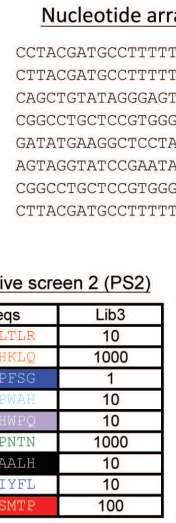

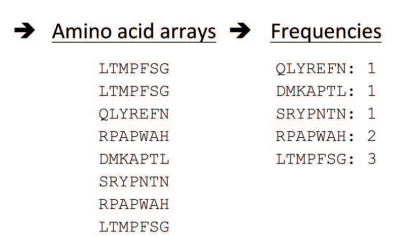
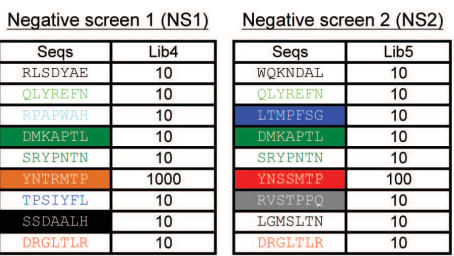

e.

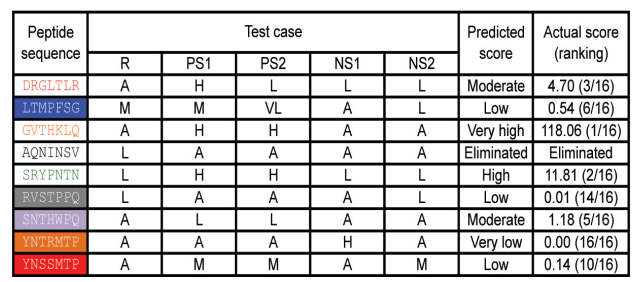

g.

\begin{tabular}{|c|c|c|c|}
\hline Screen & Total reads & Unique reads & Run time (s) \\
\hline CAF 1 & 2106522 & 628893 & 400 \\
\hline CAF 2 & 2194679 & 495842 & 414 \\
\hline CAF 3 & 2371639 & 591354 & 451 \\
\hline MRC5 & 2439664 & 679702 & 466 \\
\hline HPDE & 3098690 & 889169 & 653 \\
\hline HUVEC & 823013 & 113366 & 149 \\
\hline HUVEC-TCM & 875145 & 138044 & 159 \\
\hline BXPC3 & 1032584 & 148313 & 200 \\
\hline YAPC & 919508 & 145464 & 172 \\
\hline L3.6PL & 1238625 & 201710 & 231 \\
\hline
\end{tabular}

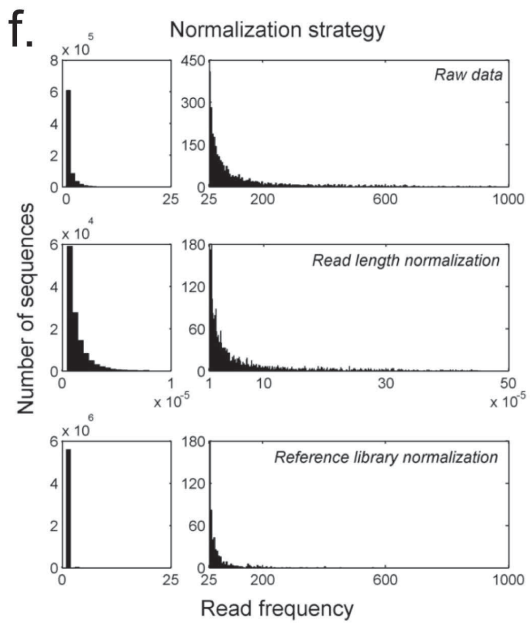

Figure 3.2: NGSanalyze. (a) Sequences obtained from the raw Illumina files. Regions flanking the displayed sequences (red) are blue and underlined. (b) A screenshot of the NGSanalyze program graphical user interface (GUI). Part 1 processes the raw data, and part 2 analyzes the data. (c) Translation of peptide sequences and calculation of read frequencies. (d) Simple libraries were created for which the appropriate scoring and ranking would be easy to calculate. (e) Predicted rankings were determined by visual inspection of each test case. For example, a peptide that is high in positive screens and very low in negative and reference screens should be predicted as an ideal peptide. Read frequencies were either absent (A), 1 (very low, VL), 10 (low, L), 100 (medium, M), or 1000 (high, H). (f) In order to allow interlibrary comparison, the data underwent two normalizations, first to read depth and then to a reference library. Data from the CAF1 screen was used to generate histograms of the entire library at each stage of the normalization process. (g) Summary of characteristics of representative screens, including the amount of time it took the NGSanalyze program to complete part 1 for each screen. 
or produced in house. Part 2 of our software generates a matrix comparing up to 20 screens of interest and subsequently sorts the matrix to cluster the most promising sequences at the top of the matrix. Simple libraries that could be visually inspected and ranked according to best candidates were processed using part 2 of our software. For each test case, we qualitatively predicted what our normalized read frequency should be and found that our software was able to match these predictions in its actual scoring and ranking (Figure $3.2 \mathrm{~d}$ and e). This aspect of the software can be extended to incorporate more screens by mimicking our provided code in a MATLAB script format (Appendix 1). Our program is able to handle all aspects of analysis from the raw data to a sorted matrix of top sequences and does not require expertise in MATLAB.

In order to enable multi-screen comparison, it was necessary to account for varying depths of sequencing between groups on the Illumina system, which we accomplished by normalizing each group to the total number of reads. This normalization altered each group by a different scaling factor and therefore did not alter the read frequency distribution within each library (Figure 3.2 f). We next used the observed frequencies of amplified, naïve, unselected $\mathrm{PhD} 7$ library (NEB) to construct a reference distribution of frequency, which approximates the frequency of each displayed peptide before selection. We normalized our post selection data to the reference distribution in order to separate sequences that reflect target binding selection pressure from those enriched by amplification rate. Because each sequence has a unique frequency in the reference library, this normalization significantly alters the read frequency distribution of sequences within each library (Figure $3.2 \mathrm{f}$ ). Reference library frequencies reflect the starting distribution of the library, amplification advantage of certain phage with faster replication rates, and the degree of binding of each displayed peptide. Normalization to the reference distribution removes the differences caused by the underlying distribution 
and amplification advantage, making it possible to identify the displayed peptides with the strongest binding. This normalization process is automated as a portion of part 2 of our software (see Appendix 1 for coding of normalization function). Thus, our program is able to rapidly produce targeted sequences with approximately seven minutes of run time per screen and about four minutes of run time to produce a 10-library matrix with 1,000 sequences (Figure 3.2 g).

\subsubsection{Purified protein screens}

As a starting point, my colleague, Dustin Bauknight, carried out a validation screen on immobilized streptavidin using the $\mathrm{PhD} 7$ library because it has an expected outcome of enriching for the HPQ amino acid motif [72]. We performed the screen in duplicate using either the specific eluant biotin or a non-specific low $\mathrm{pH}$ glycine wash to elute phage, and compared it to screens performed on immobilized vascular cell adhesion molecule (VCAM) protein and adipose tissue (Figure 3.3 a). Using our normalization and analyzation techniques, two of the top three and four of the top ten peptides had the expected HPQ motif. Further analysis of the same data set for phage specific for VCAM yielded the putative VCAM binder VRHQTSP, the highest ranked result, which mimics the well-validated VCAM binding peptides VHPKQHR and VHSPNKK [73-75]. These results demonstrated the power of our method to accurately distill the targeting peptide sequences from the rest of the data and the flexibility of our method to apply the same data in identification of targets to different proteins. We measured the correlation between experimental repeats by comparing peptide frequency from individual sequencing runs to the average peptide frequency in order to analyze the reproducibility (Figure $3.3 \mathrm{~b}-\mathrm{d}$ ). The inherent noise of phage display was demonstrated by the dispersion in read frequency of replicates in which the entire screening process was repeated and suggested that multiple screens in parallel increase the robustness of the 


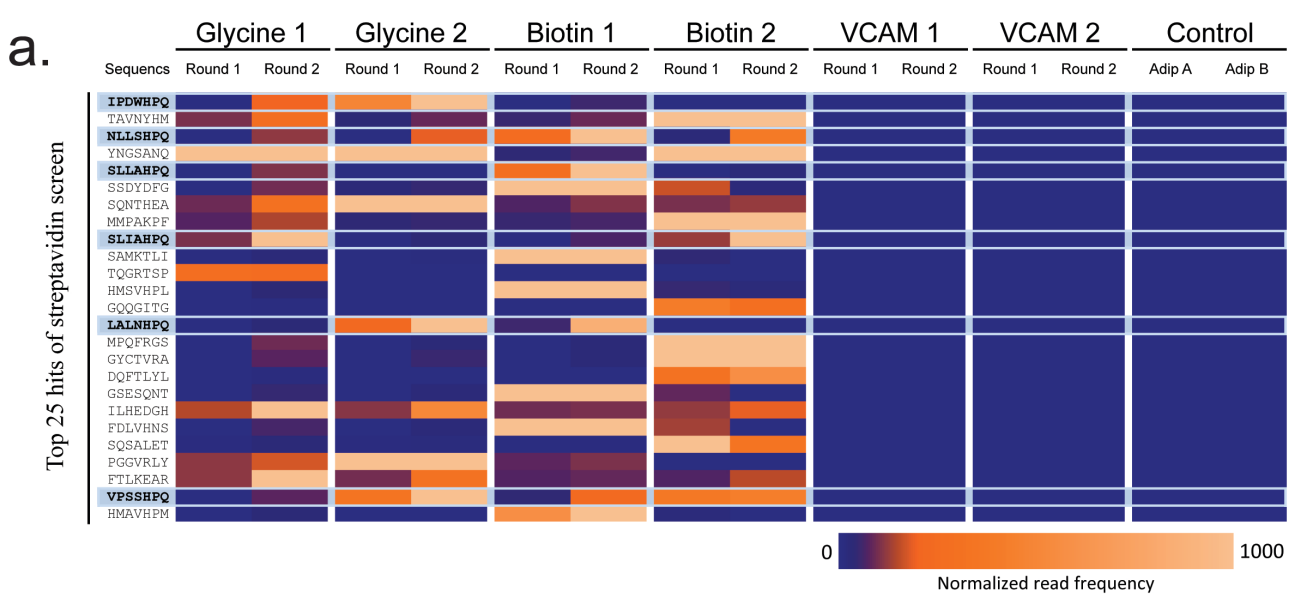

b.

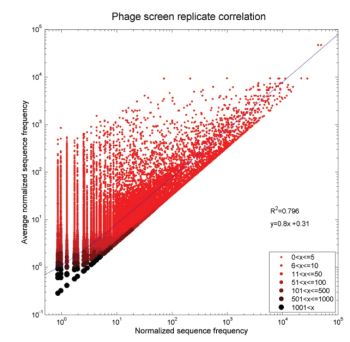

C.

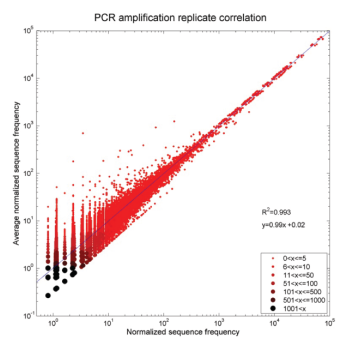

d.

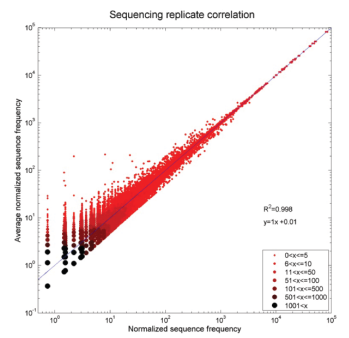

Figure 3.3: Purified protein screens. (a) The heat map represents normalized frequencies for four phage screens on streptavidin over two rounds. Screens using either glycine or biotin to elute bound phages were done in duplicate. Negative screens were completed on other purified protein and eluted with glycine. Sequences containing the expected HPQ motif are present at the top of the matrix based on ranking according to normalized frequency. Scatter plots across replicates of the entire screen (b), PCR (c), and sequencing (d).

process (Figure $3.3 \mathrm{~b}$ ). As expected, much stronger correlation was seen in replicates where only the PCR amplification step (Figure 3.3 c) or DNA sequencing step (Figure 3.3 d) were repeated. Taken together, it appears that the variability between different screens is inherent in the screening process and we attribute it to variance in the binding environment each time a biopanning is performed. Confirmation of the predictive power and robustness of our methodology led us to apply our technique to identify novel targeted peptides for a clinically relevant cell type, CAFs.

\subsection{Discussion}

The efficiency of our approach makes the discovery of cell type specific targeted ligands 
quick and easy, delivering on the expected potential of phage-based combinatorial libraries. Importantly, our approach harnesses the power of matrix-based analysis to predict which ligands will have high specificity, decreasing the lengthy validation process associated with traditional combinatorial library screening. This robust methodology quickly identifies a set of high specificity ligands selective for and against any desired cell type. Additionally, the system of comparing multiple screens ameliorates the need to do additional subtraction steps. The screening process requires only common laboratory equipment and can be performed with commercially available or custom phage libraries. The deep sequencing can be outsourced to core facilities and up to 24 libraries can be sequenced in the same run. The development of our program, NGSanalyze, automates the analysis process using a user-friendly GUI that minimalizes the need for phage display experience or MATLAB expertise. The program is optimized for use with NEB's commercially available libraries, and has the flexibility to be easily used with other libraries. The screening process typically takes four to five days and NGSanalyze can extract, translate, and sort data from raw .fastq files in minutes. Peptide synthesis can also be outsourced and, once received, liposomes can be generated in about a week. This method generates targeted ligands in a faster and less labor-intensive way than other approaches available and, notably, has a higher success rate when compared with other phage screens (see chapter 4).

We chose to repeat each positive screen in parallel. This repetition helps to account for issues stemming from the initial low copy number of each phage. Two billion phage are simultaneously screened, of which statistically, about ten phages are displaying the same sequence. With such a large number of phage competing for binding to cells, the small number of phage corresponding to a sequence with high-affinity may not have opportunity to bind to the cells because other phage are blocking sites or the peptides 
may not be present in the aliquot of the original starting library. Not only do phage exhibit a high degree of non-specific binding; but they move passively and rely on random interactions with the right target to bind. The odds of capturing the optimal random interactions are increased by repeated screens. By this logic, phage with high specificity likely will not appear at the end of every screen; however, it is encouraging to note occurrence of a sequence in more than one parallel screen.

\subsection{Methods}

\section{Biopanning}

Streptavidin biopanning was carried out according to manufacturer's instructions (NEB). Briefly, wells of a 96-well plate were coated with $100 \mathrm{ug} / \mathrm{uL}$ streptavidin and incubated overnight. $100 \mathrm{uL}$ of DBPS/1\% bovine serum albumin (BSA) containing $10 \mathrm{uL}$ of $\mathrm{PhD} 7$ library (2e11 phages) was added to each well. Plates were incubated one hour and washed five times with DPBS/1\% BSA in order to remove non-specifically bound phages. The remaining phage were eluted for nine minutes with $100 \mathrm{uL}$ glycine buffer (0.2 M glycine, $0.5 \mathrm{M} \mathrm{NaCl}, \mathrm{HCl}$ to $\mathrm{pH}$ 2.2) or for 30 minutes with biotin (o.1 $\mathrm{mM}$ ). The phagecontaining elution was immediately neutralized with $17 \mathrm{uL}$ of $1 \mathrm{M}$ Tris- $\mathrm{HCl}(\mathrm{pH}$ 9.2).

\section{Naïve reference library preparation}

$10 \mathrm{uL}$ of a 1:1000 dilution of PhD7 library (NEB) was used to create a reference library. Note: this aliquot must come from the same lot number of library due to the variability present between lot numbers [66]. This aliquot was amplified and prepared for deep sequencing in the same manner as all other libraries obtained by biopanning.

\section{PCR amplification of phage DNA}

Phage were amplified and DNA isolated according to manufacture's protocols. A master mix was kept on ice (per sample): $2 \mathrm{uL}$ universal primer (10uM, see table 1), $25 \mathrm{uL}$ 
MyTaq HS Red Mix (2x, Bioline), 19 uL MilliQ water. 46 uL of master mix was aliquoted into PCR tubes, to which the following was added: $2 \mathrm{uL}$ indexing primer (10uM, see table 1) and $2 \mathrm{uL}$ DNA template $(1 \mathrm{ng} / \mathrm{uL})$. All primers were designed to work with $\mathrm{PhD} 7$ or PhD12 libraries (NEB). Following an initial start of $95^{\circ} \mathrm{C}$ for one minute, 20 cycles of PCR were carried out as follows: $95^{\circ} \mathrm{C}$ for $30 \mathrm{~s}, 60^{\circ} \mathrm{C}$ for $30 \mathrm{~s}, 72^{\circ} \mathrm{C}$ for $30 \mathrm{~s}$. The PCR product was kept at $4^{\circ} \mathrm{C}$ until ready for purification. PCR purification was carried out using a QIAquick PCR purification kit (Qiagen), following the manufacturer's instructions. Samples were then prepared and sent to a core facility for Sanger sequencing to confirm proper indexing of the samples using PCRPrimerN FWD (10uM, Table 3.1).

\section{NGSanalyze of deep sequencing results}

Samples were sent to a core facility for deep sequencing. At the core facility, the quality and quantity of individual amplicons was assessed using Qubit fluorescence spectrophotometry in combination with an Agilent Bioanalyzer. Samples were run on an

Illumina MiSeq Sequencer using the standard protocols recommended by Illumina for MSeq, using the MiSeq 150 V3 kits. Files obtained from deep sequencing (.fastq) were entered into the part 1 of the NGSanalyze program with NEB PhD library selected and the number of amino acids per sequences entered as seven. This produced an excel file for each screen with sequences and corresponding read frequencies. Each excel file was then entered into part 2 of NGSanlyze to obtain a matrix of sorted, normalized read frequencies and corresponding sequences. A heat map was generated using Excel in order to visualize this matrix. 


\begin{tabular}{|c|c|}
\hline Index primer & Sequence $\left(5^{\prime}-3^{\prime}\right)$ \\
\hline Truseq Adapter, Index 1 & $\begin{array}{l}\text { CAAGCAGAAGACGGCATACGAGATcgtgatGTGACTGGAGTTCAGACGTGTGCTCTTCCGA } \\
\text { TCGCCCTCATAGTTAGCGTAACG }\end{array}$ \\
\hline TruSeq Adapter, Index 2 & $\begin{array}{l}\text { CAAGCAGAAGACGGCATACGAGATacatcgGTGACTGGAGTTCAGACGTGTGCTCTTCCGA } \\
\text { TCGCCCTCATAGTTAGCGTAACG }\end{array}$ \\
\hline TruSeq Adapter, Index 3 & $\begin{array}{l}\text { CAAGCAGAAGACGGCATACGAGATgcctaaGTGACTGGAGTTCAGACGTGTGCTCTTCCGA } \\
\text { TCGCCCTCATAGTTAGCGTAACG }\end{array}$ \\
\hline Truseq Adapter, Index 4 & $\begin{array}{l}\text { CAAGCAGAAGACGGCATACGAGATtggtcaGTGACTGGAGTTCAGACGTGTGCTCTTCCGA } \\
\text { TCGCCCTCATAGTTAGCGTAACG }\end{array}$ \\
\hline TruSeq Adapter, Index 5 & $\begin{array}{l}\text { CAAGCAGAAGACGGCATACGAGATcactgtGTGACTGGAGTTCAGACGTGTGCTCTTCCGA } \\
\text { TCGCCCTCATAGTTAGCGTAACG }\end{array}$ \\
\hline TruSeq Adapter, Index 6 & $\begin{array}{l}\text { CAAGCAGAAGACGGCATACGAGATattggcGTGACTGGAGTTCAGACGTGTGCTCTTCCGA } \\
\text { TCGCCCTCATAGTTAGCGTAACG }\end{array}$ \\
\hline TruSeq Adapter, Index 7 & $\begin{array}{l}\text { CAAGCAGAAGACGGCATACGAGATgatctgGTGACTGGAGTTCAGACGTGTGCTCTTCCGA } \\
\text { TCGCCCTCATAGTTAGCGTAACG }\end{array}$ \\
\hline Truseq Adapter, Index 8 & $\begin{array}{l}\text { CAAGCAGAAGACGGCATACGAGATtcaagtGTGACTGGAGTTCAGACGTGTGCTCTTCCGA } \\
\text { TCGCCCTCATAGTTAGCGTAACG }\end{array}$ \\
\hline Truseq Adapter, Index 9 & $\begin{array}{l}\text { CAAGCAGAAGACGGCATACGAGATctgatcGTGACTGGAGTTCAGACGTGTGCTCTTCCGA } \\
\text { TCGCCCTCATAGTTAGCGTAACG }\end{array}$ \\
\hline Truseq Adapter, Index 10 & $\begin{array}{l}\text { CAAGCAGAAGACGGCATACGAGATaagctaGTGACTGGAGTTCAGACGTGTGCTCTTCCGA } \\
\text { TCGCCCTCATAGTTAGCGTAACG }\end{array}$ \\
\hline Truseq Adapter, Index 11 & $\begin{array}{l}\text { CAAGCAGAAGACGGCATACGAGATgtagccGTGACTGGAGTTCAGACGTGTGCTCTTCCGA } \\
\text { TCGCCCTCATAGTTAGCGTAACG }\end{array}$ \\
\hline TruSeq Adapter, Index 12 & $\begin{array}{l}\text { CAAGCAGAAGACGGCATACGAGATtacaagGTGACTGGAGTTCAGACGTGTGCTCTTCCGA } \\
\text { TCGCCCTCATAGTTAGCGTAACG }\end{array}$ \\
\hline Truseq Adapter, Index 13 & $\begin{array}{l}\text { CAAGCAGAAGACGGCATACGAGATtgttgactGTGACTGGAGTTCAGACGTGTGCTCTTCC } \\
\text { GATCGCCCTCATAGTTAGCGTAACG }\end{array}$ \\
\hline TruSeq Adapter, Index 14 & $\begin{array}{l}\text { CAAGCAGAAGACGGCATACGAGATacggaactGTGACTGGAGTTCAGACGTGTGCTCTTCC } \\
\text { GATCGCCCTCATAGTTAGCGTAACG }\end{array}$ \\
\hline TruSeq Adapter, Index 15 & $\begin{array}{l}\text { CAAGCAGAAGACGGCATACGAGATtctgacatGTGACTGGAGTTCAGACGTGTGCTCTTCC } \\
\text { GATCGCCCTCATAGTTAGCGTAACG }\end{array}$ \\
\hline TruSeq Adapter, Index 16 & $\begin{array}{l}\text { CAAGCAGAAGACGGCATACGAGATcgggacggGTGACTGGAGTTCAGACGTGTGCTCTTCC } \\
\text { GATCGCCCTCATAGTTAGCGTAACG }\end{array}$ \\
\hline TruSeq Adapter, Index 17 & $\begin{array}{l}\text { CAAGCAGAAGACGGCATACGAGATgtgcggacGTGACTGGAGTTCAGACGTGTGCTCTTCC } \\
\text { GATCGCCCTCATAGTTAGCGTAACG }\end{array}$ \\
\hline TruSeq Adapter, Index 18 & $\begin{array}{l}\text { CAAGCAGAAGACGGCATACGAGATcgtttcacGTGACTGGAGTTCAGACGTGTGCTCTTCC } \\
\text { GATCGCCCTCATAGTTAGCGTAACG }\end{array}$ \\
\hline TruSeq Adapter, Index 19 & $\begin{array}{l}\text { CAAGCAGAAGACGGCATACGAGATaaggccacGTGACTGGAGTTCAGACGTGTGCTCTTCC } \\
\text { GATCGCCCTCATAGTTAGCGTAACG }\end{array}$ \\
\hline TruSeq Adapter, Index 20 & $\begin{array}{l}\text { CAAGCAGAAGACGGCATACGAGATtccgaaacGTGACTGGAGTTCAGACGTGTGCTCTTCC } \\
\text { GATCGCCCTCATAGTTAGCGTAACG }\end{array}$ \\
\hline TruSeq Adapter, Index 21 & $\begin{array}{l}\text { CAAGCAGAAGACGGCATACGAGATtacgtacgGTGACTGGAGTTCAGACGTGTGCTCTTCC } \\
\text { GATCGCCCTCATAGTTAGCGTAACG }\end{array}$ \\
\hline Truseq Adapter, Index 22 & $\begin{array}{l}\text { CAAGCAGAAGACGGCATACGAGATatccactcGTGACTGGAGTTCAGACGTGTGCTCTTCC } \\
\text { GATCGCCCTCATAGTTAGCGTAACG }\end{array}$ \\
\hline TruSeq Adapter, Index 2 & $\begin{array}{l}\text { CAAGCAGAAGACGGCATACGAGATatatccagGTGACTGGAGTTCAGACGTGTGCTCTTCC } \\
\text { GATCGCCCTCATAGTTAGCGTAACG }\end{array}$ \\
\hline TruSeq Adapter, Index 24 & $\begin{array}{l}\text { CAAGCAGAAGACGGCATACGAGATaangaatGTGACTGGAGTTCAGACGTGTGCTCTTCC } \\
\text { GATCGCCCTCATAGTTAGCGTAACG }\end{array}$ \\
\hline PCRPrimerN (FWD) & AATGATACGGCGACCACCGAGATCTACAC \\
\hline PCRPrimerN (REV) & CAAGCAGAAGACGGCATACGAGAT \\
\hline Universal (FWD) & $\begin{array}{l}\text { AATGATACGGCGACCACCGAGATCTACACTCTTTCCCTACACGACGCTCTTCCGATCTTTA } \\
\text { TTCGCAATTCCTTTAGTGG }\end{array}$ \\
\hline
\end{tabular}

Table 3.1: The primers used for sequence indexing prior to deep sequencing. Universal primer was used with one indexing primer for each library. PCRPrimerN (FWD) was used to prepare samples for Sanger sequencing. 


\section{Cloning into phage vector}

DNA sequences corresponding to peptides were cloned into the M13KE vector (NEB). Oligos corresponding to the following sequences were chemically synthesized (Euorfins) and ligated: (coding) [PHOS]GTACCTTTCTATTCTCACTCT...[optimized codon]...GGTGGAGGTTC, and (non-coding) [PHOS]GGCCGAACCTCCACC...[reverse complement of optimized codon]...AGAGTGAGAATAGAAAG. 10uL of 100uM coding and non-coding solutions were combined with $30 \mathrm{uL}$ of $17 \mathrm{mM} \mathrm{MgCl}_{2}$ in Qiagen $\mathrm{EB}$, heated for 10 minutes in a $95^{\circ} \mathrm{C}$ water bath, and slowly cooled to room temperature. The M13KE vector (NEB) was digested with ACC651 and EagI-HF (NEB) followed by CIP. Vector was purified using the Qiaquick kit (Qiagen) according to manufacturer's instructions with a final elution into $40 \mathrm{uL}$ of EB. T4DNA ligase, M13KE vector, annealed oligonucleotides, and MilliQ water were combined such that there was a 6:1 ratio of oligonucleotide to vector. One reaction mix was prepared without insert as a ligation control. The reactions were heat inactivated via incubation at $70^{\circ} \mathrm{C}$ for 10 minutes. Transformed cells were plated on LB-tet plates for each reaction and the positive transformation control (made with pUC19 DNA) was plated on an LB-ampicillin plate (100 ug / $\mathrm{mL}$ ampicillin). After overnight incubation $\left(37^{\circ} \mathrm{C}\right)$, colonies were picked and put in 50 uL MilliQ water. Samples underwent PCR (25 uL HS Taq red mix, 1 uL forward primer (5'-CCTTTAGTGGTACCTTTCTAT-3', Eurofins), 1 uL reverse primer (5’GCCCTCATAGTTAGCGTAACG-3', Eurofins), 18 uL MilliQ water, 5 uL DNA from plaques in water) with 1 cycle of $95^{\circ} \mathrm{C}$ for 15 minutes, followed by 40 cycles of $\left[95^{\circ} \mathrm{C}\right.$ for 1 minute, $55^{\circ} \mathrm{C}$ for 1 minute, $72^{\circ} \mathrm{C}$ for 1 minute], then 1 cycle of $72^{\circ} \mathrm{C}$ for 10 minutes. Sanger sequencing (Eurofins) was used to confirm insertion of the correct peptide. Clones with the correct sequence were amplified as described above and used in phage validation experiments. 


\section{Scatter plots}

Screens were replicated in three manners: (1) the entire screening process, (2) the PCR amplification step, or (3) the deep sequencing step. For each type of replication, frequencies across all replicates were averaged and plotted against the frequencies from each replicate using MATLAB. 


\section{Chapter 4}

Peptide to target CAF cells 


\subsection{Foreword}

Armed with a powerful technique for identifying targeted ligands that are selective and specific, we were excited to pursue targeting of CAFs. Our system seemed particularly fit for application to CAFs because there is still much that is unknown about them since they have only recently been introduced as critical to the cancer processes. Discovery of novel peptides that bind CAFs could open up new areas of biological research, as was the case with plectin, a biomarker for PDAC tumor epithelial cells that was found using phage display and overlooked with other methods such as microarray techniques [36]. The high ratio of CAF to tumor epithelial cells at early stages of disease also made them an ideal candidate for development of an imaging agent. And, as was our focus in this dissertation research, they are more accessible given their abundance, pro-angiogenic signaling, and perivascular location, so they could break through some of the drug delivery barriers that have plagued PDAC therapy.

\subsection{Introduction}

CAFs are a relatively recent area of cancer research and present a new angle for treatment. Compared to resident fibroblasts of normal tissues they are distinct in their molecular constitution, making them targetable, and distinct in their function, giving a reason to target them. Studies in animals of breast and prostate cancer revealed the ability of CAFs to transform neighboring initiated epithelial cells into cancerous cells [76]; but CAFs differ from malignant epithelial cells in that their changes do not originate from somatic mutations [77]. CAFs regulate tumor progression and communicate reciprocally with malignant epithelial cells via paracrine signaling molecules and secretion of exosomes [78]. However, the role of CAFs is complex and they are assigned both anti-tumor and pro-tumor effects, depending on their context. On the one hand, CAFs stimulate tumor cell proliferation and metastasis, increase 
extracellular matrix stiffness, are pro-angiogenic, and alter the activation state of immune cells. On the other hand, they are also believed to be capable of causing growth arrest or apoptosis of malignant epithelial cells and even contribute to an anti-tumor immune response [79]. It has already been shown that stroma therapy is not as simple as CAF depletion [8 9]. Rather, there is much to learn about CAFs and their complex role in cancer. In pursuit of greater knowledge about CAFs and development of novel therapy and imaging, we developed CAF-targeted peptides.

\subsection{Results}

\subsubsection{Targeting peptides to pancreatic CAFs}

We screened pancreatic CAFs in addition to other physiologically relevant cell types (normal fibroblast, pancreatic ductal cells, pancreatic tumor epithelial cells, normal endothelial cells, and tumor-like endothelial cells). We examined the top 40 sequences produced by our analysis program and selected only sequences with repeated exemplary binding characteristics (Figure 4.1 a). The high throughput nature of our methodology led to a high number of promising candidates. In considering how to predict the best peptide sequences, we took into account (1) the increased robustness from parallel screens that we discovered in our protein work (Figure $3.3 \mathrm{~b}$ ), (2) the similarity of amino acid side-chain patterns, and (3) the round to round progression of the clones.

Firstly, based on the screen to screen variability we found in on our work with purified, immobilized protein (Figure $3.3 \mathrm{~b}$ ), we repeated the entire screening process on CAF cells three times and examined the overlap of these screens. Because it is difficult to visualize screen overlap with more than a million sequences, we used a random number generator to look at snapshots of a random group of 200 sequences (Figure $4.1 \mathrm{~b}$ ). The reference library was also included to differentiate sequences that are ubiquitously high. Between different CAF screens, there was high variability among the low read 


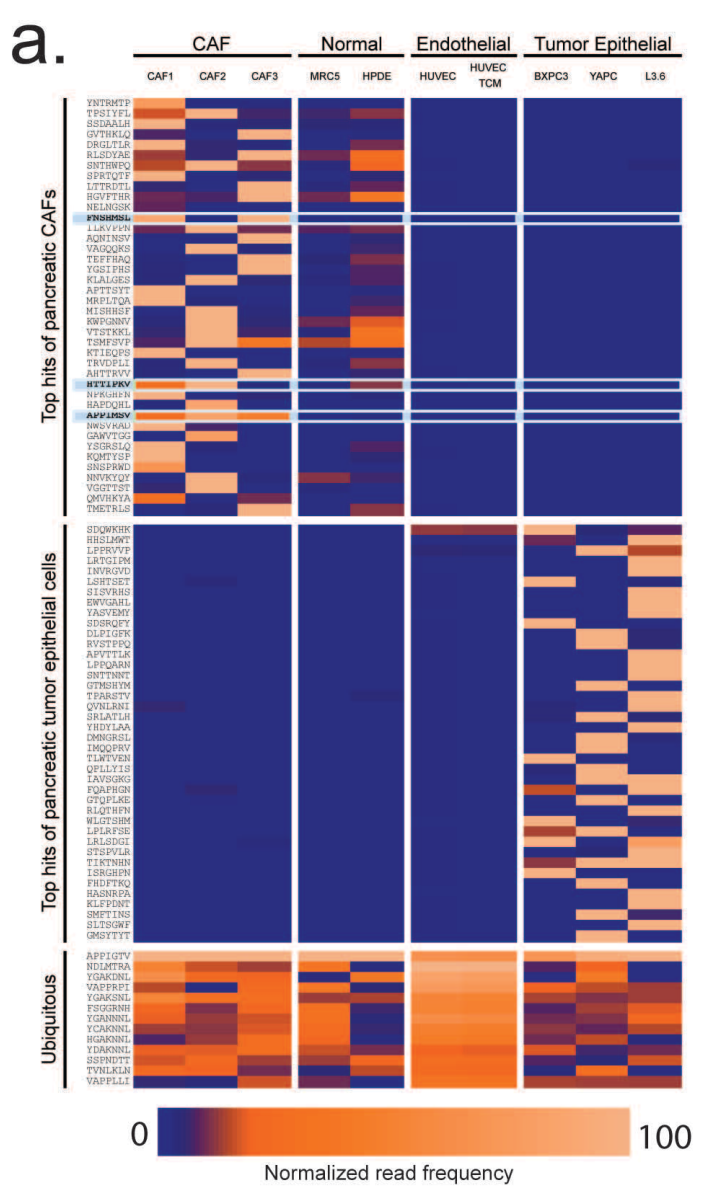

b.
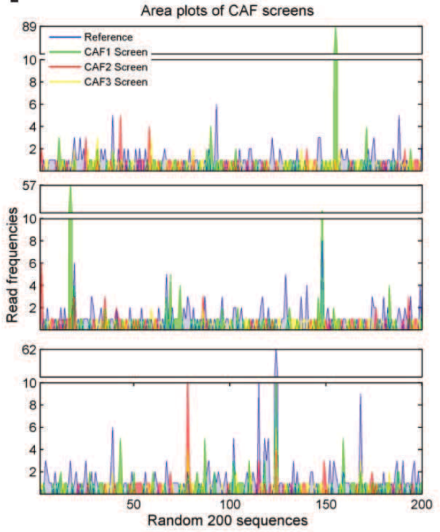

d.

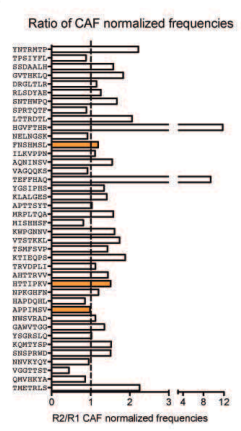

e.
C.
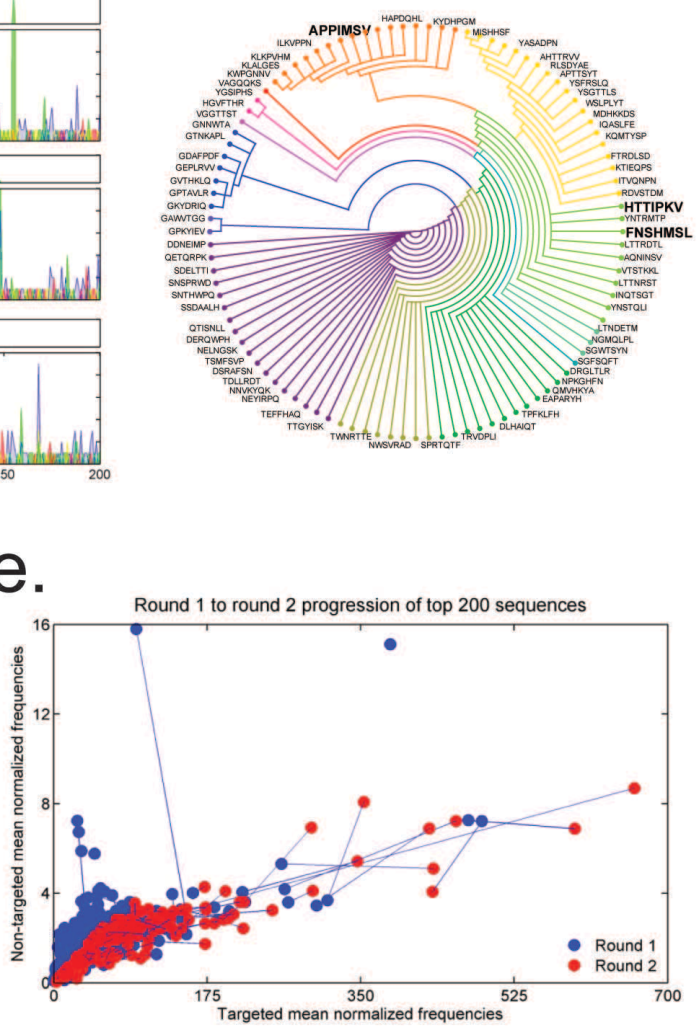

Figure 4.1: Process of choosing CAF-targeted peptide sequences. (a) A heat map visualization of matrix was generated using conditional formatting in Excel for the relevant cell types. The top 40 CAFtargeted and tumor epithelial cell-targeted sequences are labeled. Sequences that appear to bind all cell types are labeled as ubiquitous. Sequences selected for further validation are boxed in light blue and bolded. (b) A homology tree was generated for the CAF network. Patterns of the characteristics of the sidechains of the amino acids (positive, negative, uncharged, hydrophobic, or special case) within each sequence were used to group peptides into families. The network was written in standard Newick format and visualized using [105]. (c) In MATLAB, a random number generator was used to pull out 200 sequences from the three CAF screen repetitions and the reference library. The data was visualized as area plots with lightly shaded regions that show overlap between different screens. (d) The ratio of average round two CAF scores to round one CAF scores was calculated for the top 40 sequences. (e) Plots were generated using MATLAB showing the trajectory from round one (blue) to round two (red) of the top 40 sequences. The $y$-axis is the average non-targeted cell line normalized read frequency with higher frequencies indicating more binding to negative cell lines. The $x$-axis is the average CAF normalized read frequency with higher frequencies indicating more binding to the CAFs. 
frequencies $(<2)$; however, in cases where the frequency jumped to ten or greater, there was generally at least two screens in accordance (Figure $4.1 \mathrm{~b}$ ). Thus it became our primary requirement that peptide sequences show high binding in at least two of the three positive CAF screens.

Secondly, we factored in the pattern of amino acid side chain characteristics (positive, negative, uncharged, hydrophobic, or special case). Using these patterns, we created family groups that suggest similar binding since enrichment of multiple phage clones with shared characteristics lends further confidence of specific binding; we found that two of the three selected peptides fell within the same family (Figure $4.1 \mathrm{c}$ ). These family groups also enable group-based validation and provide an additional means of visualizing the results of a complicated competitive binding environment, unveiling ideal binding characteristics for targeting a certain cell type.

Thirdly, the round to round progression was important because the phage screening process enriches the library in each round of selection. Therefore, we tracked the progression of the sequences from the first to second round of biopanning and looked for stable or increasing targeted scores (Figure $4.1 \mathrm{~d}$ ). We also examined the trajectories of phage binding from round 1 to round 2 using vector plots. There was a clear general trend of a downward and outward shift, that is away from non-targeted binding and towards targeted binding, a pattern that indicates selective peptide sequences (Figure 4.1 e). In consideration of all three factors, we chose to proceed with the sequences FNSHMSL, HTTIPKV, and APPIMSV; they had high normalized frequencies across at least two out of three repeated screens, were part of families, and had the desired round to round progression. 
Two additional analyses demonstrated the versatility and robustness of our method. The versatility of our method was illustrated by re-sorting the same data to highlight sequences specific to pancreatic tumor epithelial cells (Figure 4.1 a). An additional resorting led to the discovery of "ubiquitous" binding sequences that appear with increased frequency in all screens, most notably sequences closely related to YGAKDNL (Figure 4.1 a). The robustness of our method was demonstrated by the degree of binding of the top 40 sequences to various cells lines as shown using the technique of producing area plots for a random set of 200 sequences. These area plots show the amount of overlap between various screens for the random sequences. Given that CAFs derive from normal fibroblasts and were isolated from the pancreas of PDAC patients, we hypothesized that our overlap analysis would show that phage binding to CAFs was most similar that of those cell types. Indeed, we found that phage binding to CAFs showed closest similarity to normal fibroblasts, followed by normal pancreatic ductal cells (Figure 4.2).

\subsubsection{Validation of CAF-targeted peptide sequences}

Dramatic improvement in prediction of phages that are both selective and specific was shown by comparing phage selected using our methodology to phage selected via a traditional screen in which four rounds of selection were carried out. We cloned the three sequences predicted to have the best binding into the M13 KE vector and performed an in vitro enzyme-linked immunosorbent assay (ELISA) to compare binding of the three phage we selected with our methodology to that of six clones resulting from the traditional phage display screen. While the traditional phage display screen found four phage that were selective for CAFs versus tumor epithelial cells, none of the phage were selective for CAFs over normal fibroblasts. However, two of the three phage chosen using our method, HTTIPKV and APPIMSV, had greater than 1.5-fold selectivity for CAFs 
a.
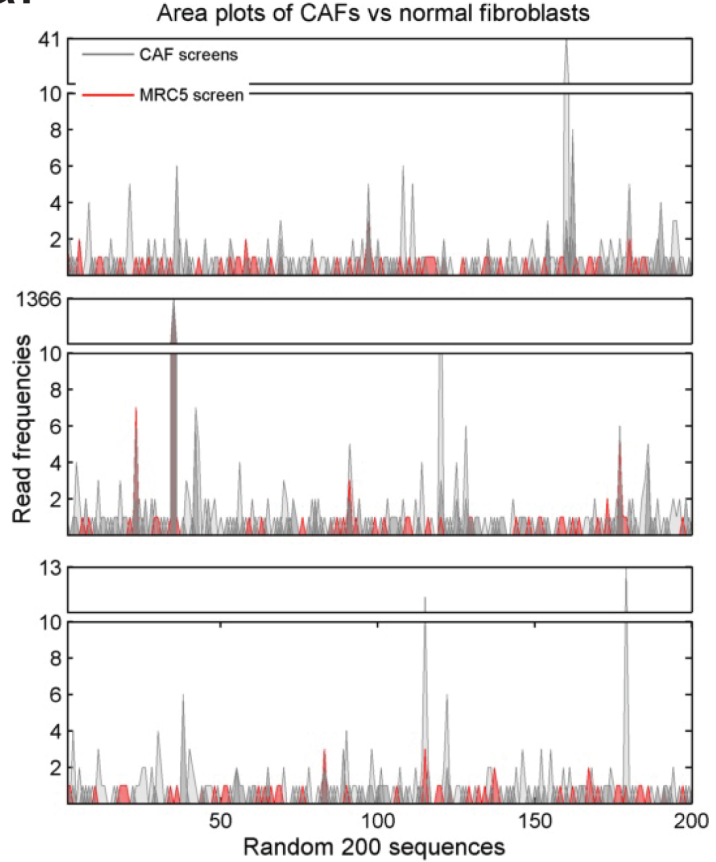

b.
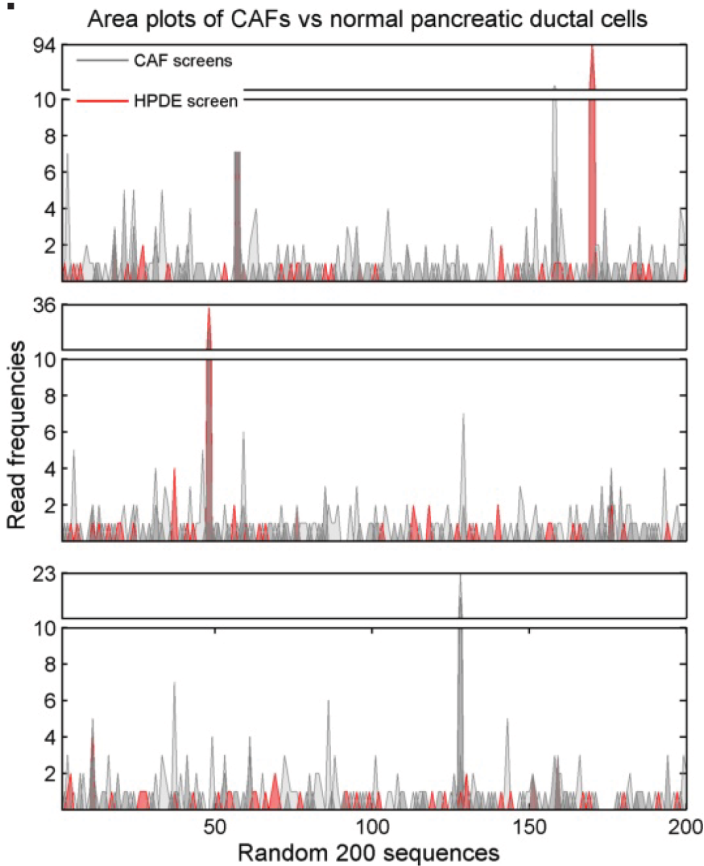

Figure 4.2: Area plot showing overlap of 200 random sequences between positive redundant screens and negative screens of normal cells. (a) In MATLAB, a random number generator was used to pull out 200 sequences from the CAF screens and the normal fibroblasts. The data was visualized as area plots with lightly shaded regions that show overlap between different screen, thus darker shades of gray indicate overlap of multiple CAF screens. (b) Area plots of three sets of 200 randomly selected sequences for the CAF screens versus normal pancreatic ductal cells.

versus normal fibroblasts and greater than 1.2-fold selectivity for CAFs versus tumor epithelial cells (Figure $4 \cdot 3$ a).

We performed two additional in vitro validations. Flow cytometric analysis indicated 2-, and 1.5-fold higher binding of clones HTTIPKV and APPIMSV, respectively, to CAFs than to wild type KE phage with no peptide displayed (Figure $4.3 \mathrm{~b}$ ). Further, HTTIPKV and APPIMSV had higher binding to CAFs when compared to normal fibroblasts (MRC5). In accordance with our ELISA data, clones HTTIPKV and APPIMSV had higher specificity whereas FNSHMSL showed minimal or no specificity (Figure 4.3 a). Secondly, we further tested our peptide sequences on human pancreatic stellate cell 
a.

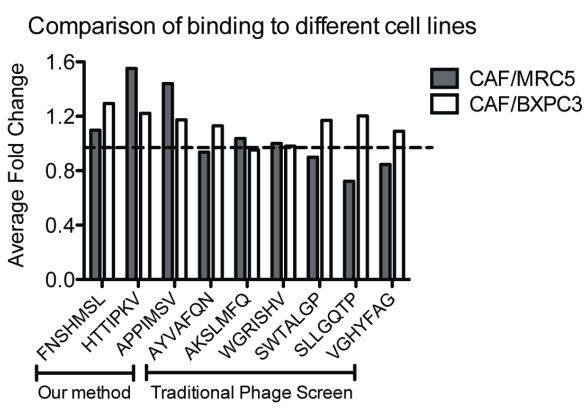

C.

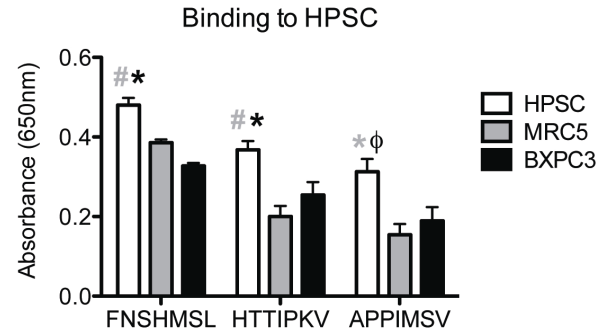

d.

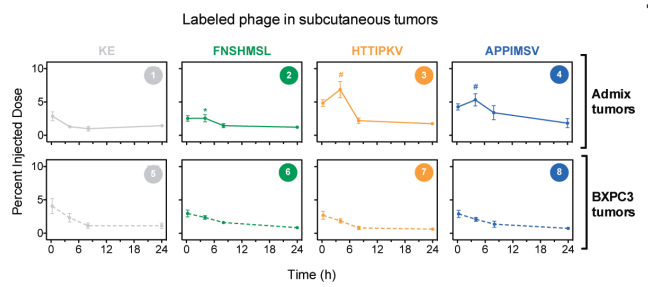

b.

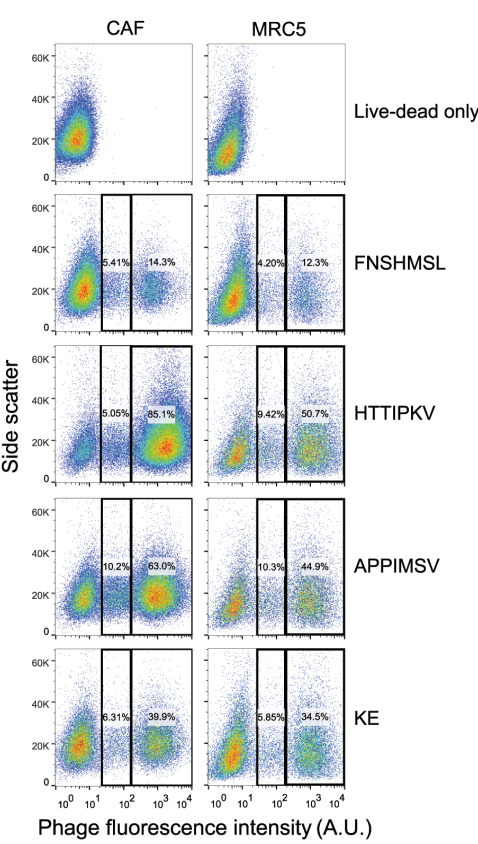

f.

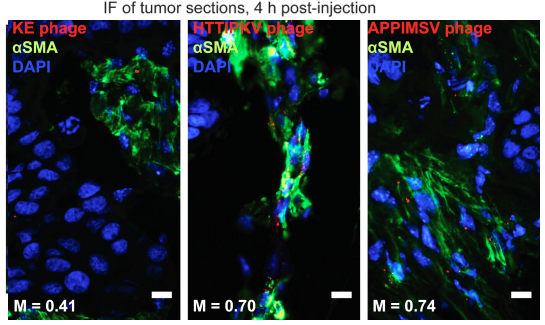

e.

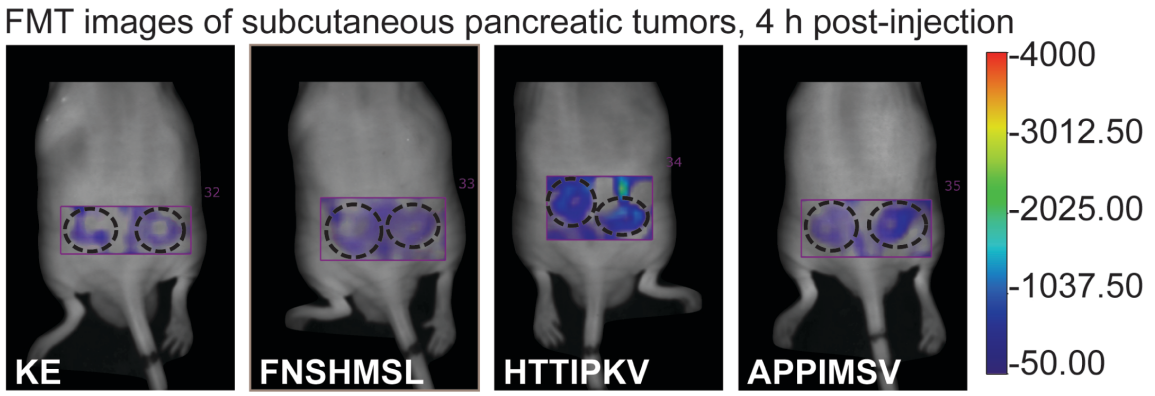

Figure 4.3: In vitro and in vivo peptide sequence validation. (a) An ELISA compares the binding of phage displaying the peptides to CAFs versus normal fibroblasts (MRC5) as well as CAFs versus tumor epithelial cells (BXPC3). The first three sequences were selected using our methodology; whereas, the next six sequences were found using a traditional phage display approach. The dashed line indicates a fold change of one. (b) Flow cytometry was performed by binding fluorescently-labeled phage to cells with a live-dead violet stain. Data was gated on cell population, live cells, and phage positive cells. (c) Phage were bound to cells, washed, and fixed. Phage amount was quantified on a plate reader by adding HRP-aM13 plll antibody and TMB. Statistical significance was measured with a student t-test between HPSC and MRC5 (gray symbols) as well as between HPSC and BXPC3 (black symbols) where $\# p<0.01$, ${ }^{*} p<0.02$, and $p<0.06$. (d) Fluorescentlylabeled phage were injected into mice bearing subcutaneous admix CAF/BXPC3 tumors or BXPC3-only tumors ( $n=6$ tumors per group) and tumor accumulation was measured on an FMT using a region-of-interest around the tumor area. Statistical significance was determined using student's t-test of each type of displayed peptide versus KE with $\# p<0.01$ and ${ }^{*} p<0.02$. (e) FMT images of mice scanned 4 hours post-injection. Tumor regions have been circled with dashed lines. (f) Tumor sections were fixed, sectioned, and stained with antiaSMA, then mounted with prolong gold anti-fade with DAPI. Mander's correlation coefficients (M) are indicated at the bottom of each image. Scale bars, 10 um. For each phage type, images are representative of three tumor sections of two tumors. 
line (HPSC), of which the majority population is CAFs, that were isolated from an independent and geographically different population of human PDAC patients than the CAFs used for screening (Figure $4.3 \mathrm{c}$ ) [7]. The selectivity of our peptide sequences for the HPSCs versus normal fibroblasts and tumor epithelial cells was demonstrated using this independently isolated cell line.

Our in vivo targeting validation consisted of systemic injection of the fluorescentlylabeled phage into nude mice bearing subcutaneous $\mathrm{BXPC} 3 / \mathrm{CAF}$ admix tumors or BXPC3-only tumors on their flanks and analysis by fluorescence molecular tomography (FMT). All three chosen sequences exhibited a classic pharmacokinetic curve on admix tumors; whereas, wild type showed no accumulation phase (Figure $4.3 \mathrm{~d}$, graphs 1-4). In BXPC3-only tumors, a clearance phase was observed for all phage and they overlapped with M13 KE (Figure 4.3 d, graphs 5-8). Phage accumulation was highest at four hours where HTTIPKV and APPIMSV sequences had 3- and 2-fold higher binding, respectively (Figure $4.3 \mathrm{~d}$ and e). Immunofluorescent images of tumor sections from admix tumor mice sacrificed at $4 \mathrm{~h}$ show binding of HTTIPKV and APPIMSV phage to the stroma as assessed by a CAF marker, $\alpha$-smooth muscle actin ( $\alpha$ SMA), with minimal binding present for wild type KE phage (Figure 4.3 f). Using the JACoP plugin (http://rsb.info.nih.gov/ij/plugins/track/jacop.html, [8o]) in ImageJ, an average mander's correlation coefficient was calculated for 10 field views of three tumor sections of two tumors (6o images total) between the phage and aSMA-positive areas (representative images, Figure 4.3 f). The mander's coefficient was high for both HTTIPKV (o.70) and APPIMSV (0.74) and low for KE (0.41) indicating phage clone binding to aSMA-positive cells. To assess our subcutaneous model, we looked at hematoxylin and eosin (HE) staining of each type of tumor to validate that the admix injection was producing a tumor 

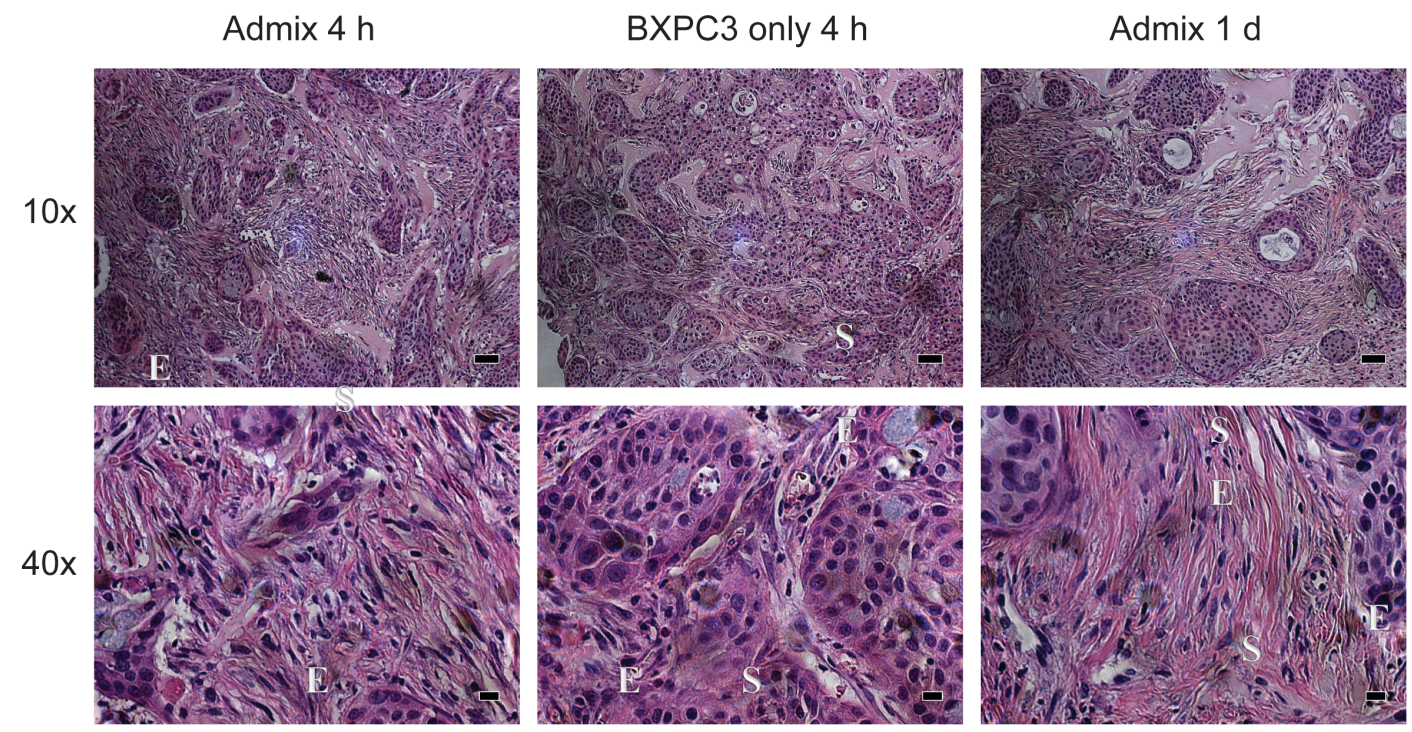

Figure 4.4: Epithelial content of subcutaneously injected tumors. (a) Light microscope images of HE stained admix CAF/BXPC3 or BXPC3 only tumor sections show stromal (S) and epithelial (E) compartments. 10x scale bar (black), 50 um. 40x scale bar (black), 10 um.

with a higher stromal content (Figure 4.4). Taken together, these data indicate the specificity of the phage clones for the CAF cells.

\subsection{Discussion}

Molecularly targeted affinity ligands are playing an increasingly important role in pharmaceutical discovery and development and the design of molecular imaging agents. Indeed, efficacy of drugs is dependent on the therapy to toxicity ratio. Toxic side effects can be dose limiting, causing an otherwise efficacious drug to fail, decreasing quality of life for the patient, or premature arrest of a treatment regimen. As a representative $\mathbb{S}$ case, cancer patients receiving the molecular therapy FOLFIRINOX often had their dose reduced because of low blood counts, fever, infection, diarrhea, weight loss, and fatigue. In addition, severe adverse events required more drastic intervention such as splenectomy, in order for patients to continue treatment [81]. Thus there is an unmet clinical need to provide a means of improving the 
tolerability of drugs, which has inspired development of targeted drug delivery. However, identifying appropriate targeting moieties remains time consuming. We were able to identify potential targeted peptides to CAFs in a very short timeline: biopanning in one week, deep sequencing at a core facility in four days, and analysis in less than a day. Post-identification of potential sequences in the traditional phage display process, we typically experience a high-failure rate. In fact, we have completed entire screens that resulted in all non-specific binding phage. Thus, the success rate with our method in the context of CAFs-two out of three chosen potential targeted sequences went on to successfully validate in vitro and in vivo-served to highlight the value of the predicative power added by our quantitative analysis.

In our analysis, we elected to validate our potential targeted peptides with two types of in vitro analysis. Our primary method was ELISA, which assessed the selectivity of phage by calculating a fold change between the targeted cell line (CAFs) and other negative cell lines (Figure 4.3 a). We used mrc5, a normal fibroblast cell line because CAFs are believed to be derived from normal fibroblasts and were very similar to them in our area plot analysis (Figure 4.2 a). However, the mrc5 cell line originally came from the lung, so we also thought it was important to test our potential sequences against a pancreatic cell line. Since CAFs and tumor epithelial cells are known to communicate extensively and share some receptors possibly transferred from one to the other via exosomes [82], we also verified that our sequences were selective for CAFs over pancreatic tumor epithelial cells. In anticipation of our in vivo validation studies, we chose to compare CAFs versus the tumor eptithelial cells BXPC3s, which we planned to use to create our admix tumors. As a follow-up to this evaluation of the selectivity of the potential sequences, we also looked at flow cytometry. 
ELISAs provide a fast and inexpensive means of validating sequences; however, they are unable to differentiate between binding to a secreted substance from the cells or something present on the cell surface. This was particularly important to our application because CAFs are responsible for the deposition of large quantities of extracellular matrix and known to secrete numerous signaling molecules [34]. By contrast, flow cytometry analyzes the cells in suspension and determined that our two peptides that performed well in the ELISA were binding to cell surface targets on the CAFs. As an additional layer of robustness, we also examined a separate cell line comprised primarily of CAFs, namely the HPSCs, to ensure that the potential peptides were not specific only to one population of patients.

While in vitro confirmation of peptide sequence selectivity is promising, it is not always indicative of how the peptides will act in vivo because cells are out of their physiologic context. For this reason, it is important to also test the peptide sequences in vivo. In this case, two of the potential sequences (HTTIPKV and APPIMSV) that passed our in vitro inspection continued to perform well in vivo, while the other potential sequence (FNSHMSL) presented less favorably in all evaluations.

All of the in vitro and in vivo analyses to this point-ELISAs, flow cytometry, and subcutaneous mouse model-evaluated the peptide sequences using bacteriophage display. By cloning the peptide sequences into bacteriophage, we were able to generate an endless supply of peptide displayed on the phage for our testing purposes. Phage amplify easily in bacteria and because the displayed peptide is genetically incorporated, DNA sequencing can confirm display of the proper peptide. However, the use of display in phage for validation is not without drawbacks. Phage are "sticky," in other words, they have high non-specific binding, which varies between cell types. To minimize the nonspecific interactions between the phage particles and substrates, we avoided the use 
of Tween-20 [83]; however, there was still non-specific phage binding. This was especially evident in our flow cytometry analysis as illustrated by the KE control that had about $40 \%$ binding (Figure $4.3 \mathrm{~b}$ ). The use of wild-type KE phage was, therefore, a critical control to include; KE phage still exhibit non-specific binding, but have no specific binding because they have no displayed peptide.

Another important feature of peptides displayed on phage is that there are five copies of the peptides on each phage, creating an avidity effect. Thus, the displayed peptide may not have the same level of binding once it is taken out of the phage context and synthesized. This is an important consideration when developing a peptide imaging agent. We believe that the ability of our method to better predict the best candidate phage will be an asset in finding a sequence that can still show high binding independent of the avidity effect. In our application, using the peptides to target liposomes, this was not a concern because the liposomes are decorated with multiple copies of the peptide, keeping the avidity effect in tact.

\subsection{Methods}

\section{Cell lines}

CAFs (gift from Diane Simeone), YAPCs (ATCC), and BXPC3s (ATCC) were grown in RPMI medium 1640 (Life Technologies). MRC5s (ATCC) were grown in MEM medium (Life Technologies). HPSCs (gift from Rosa Hwang) and L3.6pls (ATCC) were grown in DMEM (Life Technologies). The RPMI, DMEM, and MEM media were supplemented with fetal bovine serum (10\%), L-glutamine (2 mM), penicillin (100 units/mL), and streptomycin (100 ug/mL). HPDEs (gift from Craig Logsdon) were grown in keratinocyte serum-free media with accompanying supplements (Life Technologies). HUVECs (LONZA) were grown in EBM supplemented with an EGM bullet kit (LONZA). Tumorconditioned media (TCM) was harvested from L3.6 cells following $48 \mathrm{~h}$ of growth to 
$90 \%$ confluency, and concentrated with Amicon 1ok centrifuge filter. Concentrated media was added to the TCM HUVECs at a final concentration of $1 \mathrm{x}, 36 \mathrm{~h}$ prior to phage screening. For biopanning, cells were grown in a row of a 96-well plate in 1:2 serial dilutions and allowed to grow in a humidified, $37^{\circ} \mathrm{C}, 5 \% \mathrm{CO}_{2}$ incubator. Plates were then examined under a microscope and wells with 70\% confluence were used for biopanning. For ELISA experiments, 50,000 cells were plated in each well of two rows of a 96-wellplate and allowed to grow in a humidified, $5 \% \mathrm{CO}_{2}$ incubator overnight.

\section{Biopanning}

Cells were prepared as described above. Media was aspirated from well and cells were rinsed with DPBS (HyClone, with calcium and magnesium). $100 \mathrm{uL}$ of DBPS/1\% BSA containing $10 \mathrm{uL}$ of $\mathrm{PhD} 7$ library (2e11 phages) was added to each well. Plates were returned to the humidified, $5 \% \mathrm{CO}_{2}$ incubator. After one hour, plates were removed and the supernatant was aspirated from the wells. Cells were washed five times with DPBS/1\% BSA in order to remove non-specifically bound phages. The remaining phage were eluted for nine minutes with $100 \mathrm{uL}$ glycine buffer (0.2 M glycine, $0.5 \mathrm{M} \mathrm{NaCl}, \mathrm{HCl}$ to $\mathrm{pH}$ 2.2). The phage-containing elution was immediately neutralized with $17 \mathrm{uL}$ of $1 \mathrm{M}$ Tris-HCl ( $\mathrm{pH}$ 9.2). Naïve reference library preparation, PCR amplification of DNA, NGSanalyze of deep sequencing results, and cloning into phage vector were carried out as described in chapter 3 methods.

\section{In vitro phage-based validation}

1-step ultra tetramethylbenzidine (TMB)-ELISA substrate solution (ThermoFisher Scientific) was allowed to warm to room temperature. Cells were prepared as described above (see Cell Lines). Cells were washed with DPBS+/1\%BSA, $100 \mathrm{uL}$ per well. For each type of phage and control wild-type KE phage, three wells were incubated with $40 \mathrm{uL}$ of the phage (10e8 phages/uL). Phage were incubated on cells for one hour in a humidified, 
$37^{\circ} \mathrm{C}, 5 \% \mathrm{CO}_{2}$ incubator. Cells were then washed three times (DPBS+/1\%BSA, $100 \mathrm{uL}$ ) and fixed (100 uL of 2\% PFA, 5 minutes), then washed twice more. HRP- $\alpha$ M13 pIII monoclonal antibody (100 uL, 1:3000 dilution in DPBS+/1\% BSA (NEB)) was added to each well for 1 hour. Cells were washed four more times and $100 \mathrm{uL}$ of TMB was added. After the TMB reacted with the HRP, the absorption was measured on a microplate reader (Molecular Devices) at $650 \mathrm{~nm}$. Flow cytometry was performed by binding fluorescently labeled phage (Vivotag 645, PerkinElmer [84]) to CAF or MRC5 for 30 minutes. Cells were washed three times and live-dead violet stain (Life Technologies) was added. Data was gathered on Beckman Coulter CyAN ADP LX and gated on cell population, live cells, and phage positive cells using FlowJo software.

\section{In vivo phage-based validation}

6- to 8-week old nude mice (Harlan) were anesthetized with isoflurane and injected with a total volume of $100 \mathrm{uL} /$ tumor containing 2 million cells. For BXPC3-only tumors, 2 million BXPC3 cells were combined 1:1 with Matrigel (BD Biosciences). For admix tumors, 0.5 million BXPC3 cells and 1.5 million CAF cells were combined 1:1 with Matrigel. Two tumors were subcutaneously injected per mouse, on each flank, and allowed to grow for 2.5 weeks before the start of the study. Eight groups total were used for this study: admix KE, admix FNSHMSL, admix HTTIPKV, admix APPIMSV, BXPC3 KE, BXPC3 FNSHMSL, BXPC3 HTTIPKV, and BXPC3 APPIMSV.

Phage displaying targeted peptides and control wild-type KE phage were labeled as previously described [84]. Briefly, fluorescent dye (Vivotag 680, PerkinElmer) and amplified phage were incubated and free dye removed using PEG precipitation and microfugation (14,000 rpm, 10 minutes). The phage were suspended in $1 \mathrm{~mL}$ of DPBS, PEG-precipitated again, and suspended in $200 \mathrm{uL}$ DPBS. Mice received tail vein injections equal quantities of dye-labeled phages. At various time-points, isofluorane- 
anesthesized mice were imaged in an FMT system (PerkinElmer). Images were reconstructed using the FMT software and regions of interest (ROI) were drawn around the tumor areas to quantify the amount of dye (pmol) present in that area. At the end of the study, organs and tumors were excised and measured on the FMT to determine the biodistribution.

\section{Immunofluorescence}

Excised tumors were divided in half to allow for immunoflurescence (IF) and immunohistochemistry (IHC). To prepare tumor tissue for IF, the tumor was immersed in $4 \%$ paraformaldehyde for 20 minutes, submerged in optimal cutting temperature compound (OCT, Tissue-tek), and frozen with liquid nitrogen vapors. Frozen cassettes were immediately placed at $-80^{\circ} \mathrm{C}$ for storage. Frozen tissues were cut using a cryostat, with sections 8um thick. OCT was removed from tumor sections frozen to glass microscope slides by submersing the slide in DPBS at room temperature for 5 minutes. Sections were then blocked for 30 minutes with $1 \%$ fish skin gelatin (FSG), rinsed twice with DPBS, stained with FITC-conjugated $\alpha$ SMA (1:250, Sigma) for 40 minutes, and rinsed five times with DPBS. Microscopy was performed using an Olympus fluorescence microscope with a 60x oil immersion objective (1.25 NA) and QImaging Retiga 200oR camera with accompanying software.

\section{Immunohistochemistry}

To prepare tumor tissue for IHC, tumors were immersed in 10\% formalin for 20 minutes and then in $70 \%$ ethanol for storage. Fixed tissue was given to the UVA Cardiovascular Research Center histological service, where the tissues were paraffin-embedded, sectioned, and stained with HE. Microscopy was performed using a Zeiss light microscope with either a 10x (0.25 NA) or 40x (0.65 NA) objective and Zeiss AxioCam MRc camera with accompanying software. 
Chapter 5

CAF-targeted

liposomes deliver CXCR2 inhibitor 


\subsection{Foreword}

With the goal from the outset of using peptides that we found in a therapeutic setting, we needed a means of employing them for drug delivery. Nanoparticles were a natural choice because we were working in cancer, where the size of nanoparticles can have a great effect on drug delivery. We were fortunate to have the expertise of Sasha Klibanov and Ryo Suzuki to help us develop a protocol for generation of liposomes that incorporated our peptides. A postdoctoral researcher in the Kelly lab, Siva Sai Krishna Dasa, worked to optimize peptide-conjugated liposomes in a cardiovascular context [85]. Building off of his work, we were able to make liposomes with the CAF-targeted peptides and apply them in a cancer setting. Using dye, we were able to see profound effects on AUC with targeted liposomes and proceeded to test drug delivery in a pilot study. We found that CXCR2 inhibitors act through CAFs to cause anti-tumor effects and believed we could augment the disruption of tumor-stromal interactions caused by free administration of CXCR2 inhibitors by delivering them with our CAF-targeted liposomes. Thus, following generation of targeted liposomes, we set out to compare the effects of no drug, free drug, and drug encapsulated in non-targeted or HTTIPKVliposomes.

\subsection{Introduction}

Targeted nanoparticles are engineered to deliver a payload to a specific cell or tissue via chemical interaction with molecules on the surface of the target area. Liposomes are a class of nanoparticles that are often used therapeutically because they are biocompatible, biodegradable, have low toxicity, do not activate the immune system, and can carry hydrophobic or hydrophilic drugs [86]. Liposomes consist of a sphere-shaped vesicle, typically formed by a phospholipid mono- or bilayer. Hydrophobic drugs can be incorporated into their lipid layer and hydrophilic drugs can be contained within their 
aqueous center. Their pharmacokinetics can be altered by surface modification and they are especially applicable to cancer therapeutics because their size allows them to exhibit the EPR effect, whereby they preferentially leak through the tumor vasculature as they circulate. This EPR effect is enhanced by addition of a targeting moiety that increases retention of the liposomes in the tumor area [86]. We incorporated our CAF-targeted peptides into nanosized liposomes with a hydrophobic dye to track them and tested their pharmacokinetics in the subcutaneous pancreatic tumors of mice. Next, we undertook a proof of concept study of a drug loaded into the liposomes.

In consideration of which drug to use in the pilot study, we focused on the fact that CAFs affect tumor characteristics by reciprocal communication with tumor epithelial cells via paracrine signaling [78]. Such tumor-stromal interactions have been shown to promote many of the lethal aspects of cancer such as tumor progression, metastasis, decreased immune response, and chemoresistance [15] and therefore the paracrine signaling molecules that enable these interactions present a tempting target for stromal therapy. An ideal candidate for a pilot study for drug delivery using our CAF-targeted liposomes would be a paracrine signaling molecule that had already been tested in its free drug form, but could benefit from incorporation into a targeted nanoparticle vehicle. Inhibition of the chemokine-CXCR2 axis was shown in mouse models to decelerate tumor growth, extend survival, and have an anti-angiogenesis effect [11]. However, systemic injection of the drug also disrupts neutrophil chemotaxis and activation, which is non-ideal for patients that generally already have a compromised immune system [12]. We hypothesized that our CAF-targeted liposomes, which we had shown to have a beneficial biodistribution, could recapitulate the effects of the free CXCR2 inhibitor while mitigating some of the off-target side effects that cause problems clinically. 


\section{$5 \cdot 3$ Results}

\subsubsection{Evolution into a targeted drug-delivery system.}

To achieve drug carrying capacity, we synthesized our leading sequences HTTIPKV and APPIMSV as peptides using standard Fluorenylmethyloxycarbonyl chloride (FMOC) chemistry, conjugated them to maleimide-polyethylene glycol(PEG, 3.4k)distearoylphophatidylethanolamine (DSPE) via the $-\mathrm{SH}$ group on the c-terminal cysteine, and subsequently incorporated them into liposomes (Figure 5.1 a). The liposomes also consisted of dioleoylphosphatidylcholine (DOPC), cholesterol, DSPE-PEG (2K), and the lipophilic dye 1,1'-Dioctadecyl-3,3,3',3'-tetramethylindotricarbocyanine iodide (DiR). Nanosight analysis of HTTIPKV-, APPIMSV-, and no peptide-liposomes showed a mean size of $98.4 \pm 0.8,122.9 \pm 1.2$ and $97.6 \pm 0.8 \mathrm{~nm}$, respectively, and mean concentration of $2.17 \mathrm{e} 13 \pm 8.57 \mathrm{e} 11,2.53 \mathrm{e} 13 \pm 5.46 \mathrm{e} 11$, and $1.95 \mathrm{e} 13 \pm 2.38 \mathrm{e} 12 \mathrm{particles} / \mathrm{mL}$, respectively (Figure $5.1 \mathrm{~b}$ ).

\subsubsection{Validation of targeted liposomes}

Injection of the fluorescently labeled liposomes into nude mice with subcutaneous $\mathrm{BXPC}_{3} / \mathrm{CAF}$ admix tumors demonstrated their targeting ability (Figure $5.1 \mathrm{c}$ ). We fit a compartment model to our liposome time course data in order to examine the tissue (tumor) pharmacokinetics (Figure $5.1 \mathrm{~d}$ ) and calculated accumulation and elimination rates for liposomes within the tumor tissue (Figure 5.1 e). Because the liposomes contained a lipophilic dye incorporated into the liposome itself, we could establish liposome location and state of decomposition (Figure 5.1 f). Liposomes tend to collect in blood-filtering organs; high accumulation of liposomes occurred in the liver and spleen and some accumulation was seen in the kidneys (Figure $5.1 \mathrm{~g}$ ). In all, estimated total drug exposure would increase dramatically using our targeted liposomes as the area- 
a.

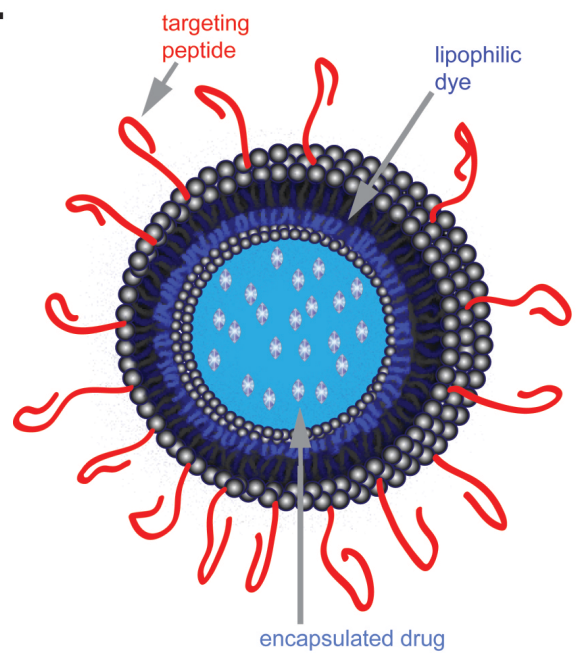

b

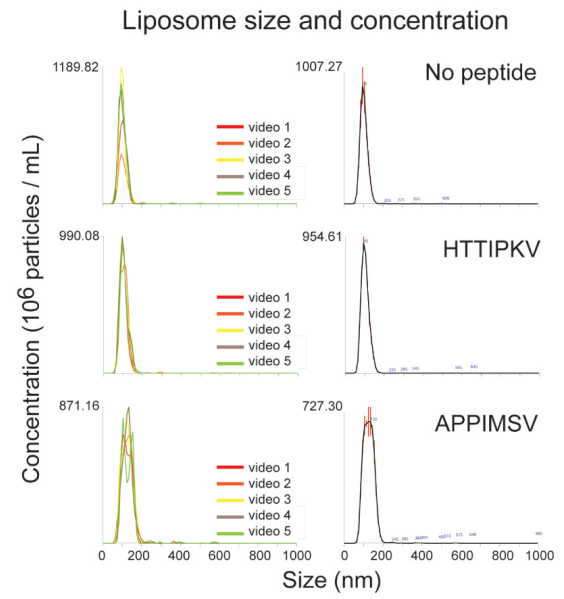

f.

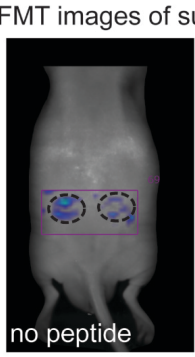

C.

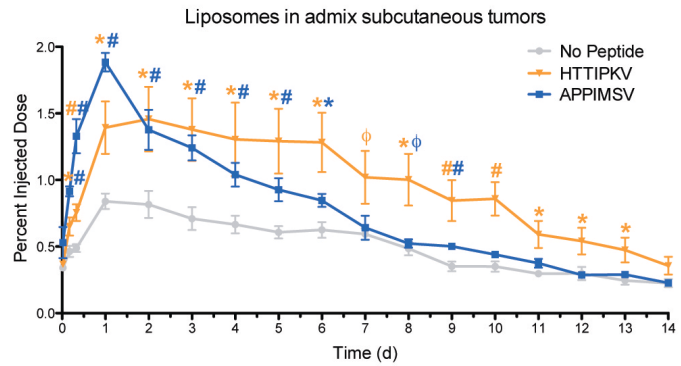

d.

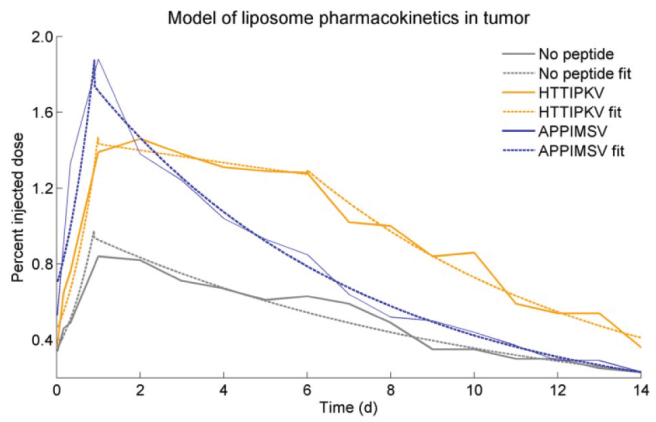

e

\begin{tabular}{|c|c|c|c|}
\hline \multicolumn{4}{|c|}{ Tumor pharmacokinetics } \\
\hline & AUC & $\mathrm{k}_{\mathrm{a}}$ & $k_{e}$ \\
\hline No peptide & 7.19 & 1.15 & -0.11 \\
\hline HTTIPKV & 13.89 & 1.20 & $\begin{array}{c}-0.02 / \\
-0.14\end{array}$ \\
\hline APPIMSV & 10.91 & 1.12 & -0.16 \\
\hline
\end{tabular}

g.

Liposome biodistribution in admix mice, $1 \mathrm{~d}$

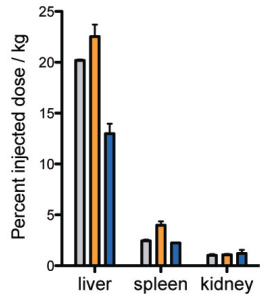

Figure 5.1: Validation of peptide-conjugated liposomes. (a) A diagram showing how targeted peptides, lipophilic dye, and drug are incorporated into a liposome. (b) The NanoSight was scripted to take five videos of the liposomes. The accompanying analysis software determined the average size and concentration of liposomes in these videos. (c) Mice bearing subcutaneous admix CAF/BXPC3 tumors ( $n=10$ tumors per group) were injected with dye-labeled liposomes and tumor accumulation was measured on an FMT using a region-of-interest around the tumor area. Statistical significance was measured with a Student t-test between liposomes with and without a peptide where $\# p<0.01,{ }^{*} p<0.02$, and $\phi p<0.06$. (d) Tumor pharmacokinetics were determined by fitting the liposome time course data with compartment models by regression analysis of log data in MATLAB. (e) Table of tumor pharmacokinetics with AUC (area under the curve), $\mathrm{K}_{\mathrm{a}}$ (accumulation exponent), and $\mathrm{K}_{\mathrm{e}}$ (elimination exponent(s)). (f) FMT images of mice one day post-injection. (g) A biodistribution was generated by sacrificing two mice per group one day post-injection. Organs and tumors were excised and the amount of dye measured via FMT. 
under-the-curve (AUC) was 1.9-, and 1.5-fold higher for HTTIPKV and APPIMSV, respectively, than for the liposome without a targeting peptide.

\subsubsection{CXCR2 inhibitor decelerates tumor growth}

Treatment of subcutaneous tumors containing both pancreatic tumor epithelial cells and CAFs with a CXCR2 inhibitor has been previously shown to cause a significant growthinhibitory effect. To address whether the CAF-targeted liposomes loaded with a CXCR2 inhibitor (SB225002) could reproduce this effect, we performed subcutaneous injections into nude mice with a mixture of $\mathrm{BXPC}_{3}$ cells and CAFs and compared the in vivo growth with intraperitoneal (IP) SB225002 injection, SB225002-loaded liposomes (HTTIPKV-targeted and non-targeted), or no treatment. We followed the same dosage schedule as previously shown to cause growth inhibition [11]. In order to circumvent

Batch 1
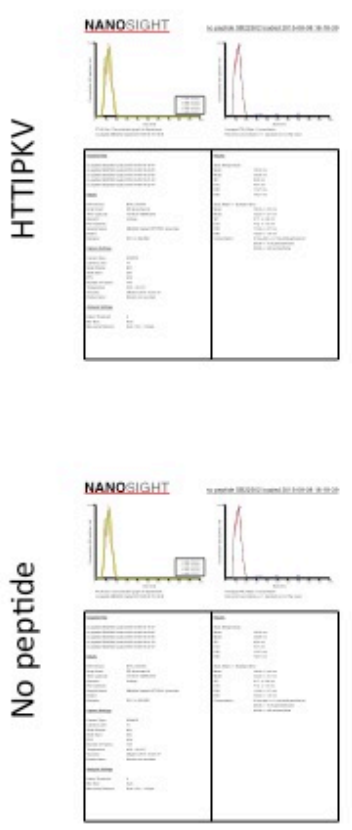

Batch 2
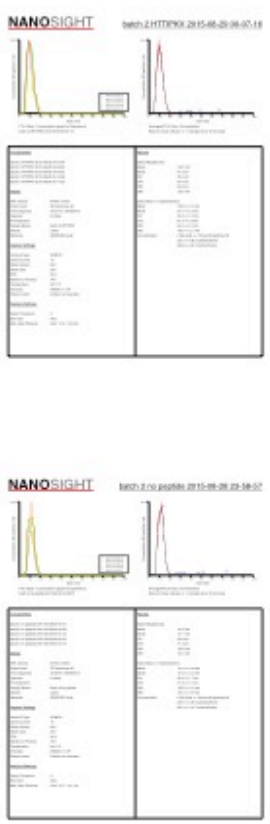

Batch 3
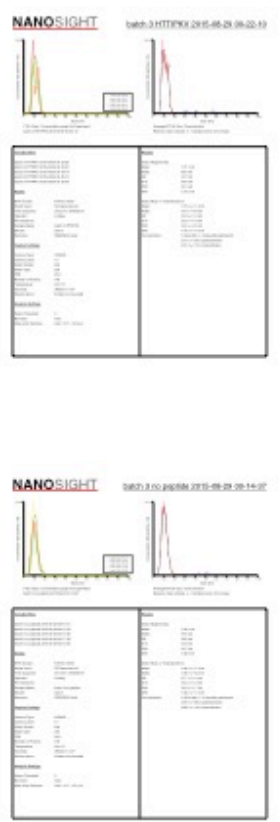

Batch 4

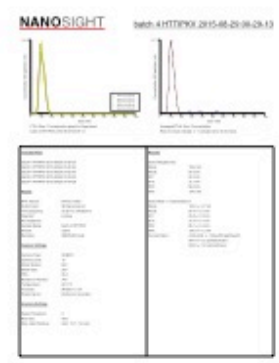

Figure 5.2: Liposome sizes and concentrations. A fresh batch of liposomes was prepared weekly and analyzed via Nanosight for mean size and concentration. Because tumors on mice injected with the no peptide-liposomes began to ulcerate and had to be sacrificed before the last week, only three batches of no peptide-liposomes were prepared. 


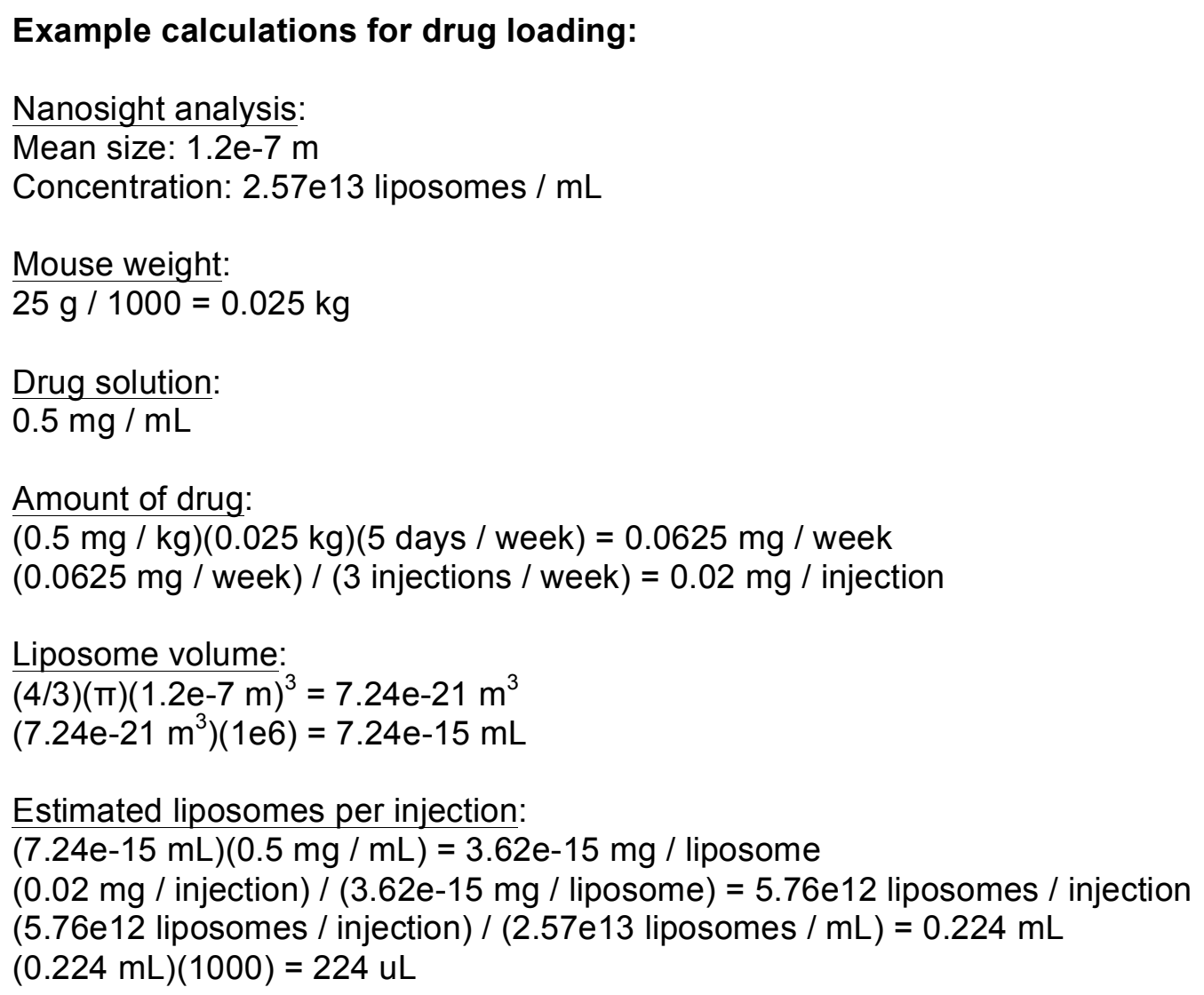

Box 5.1: Drug-carrying capacity of liposomes. The amount of SB225002 inside each liposome was estimated using the Nanosight analysis and the volume of liposomes per injection calculated in order to administer the same amount of SB225002 each week as with the IP injected mice.

potential leakiness of liposomes, which can develop over time, we produced fresh batches of liposomes weekly. To deliver the same amount of drug with liposomes as with IP injections, we measured the mean liposome size and concentration via Nanosight (Figure 5.2), calculated the average amount of drug trapped inside each liposomes, and determined the volume of liposomes necessary per injection to achieve the same weekly amount of SB225002 (Box 5.1).

Mice from all groups treated with SB225002 displayed significant deceleration in tumor growth compared to the untreated mice by day 12 (Figure 5.3 a). The greatest difference in tumor growth was observed in the mice treated with the HTTIPKV-liposomes, 
followed by the IP-injected mice, and lastly the non-targeted liposomes. Ulceration of tumors on the mice that received no peptide-liposomes caused the mice to be sacrificed before the final two treatments; no ulceration was experienced by mice in any of the other groups. By day 16, the tumor growth difference was no longer significant between the no peptide-liposome and no treatment groups. At the end of the study (day 19), significant growth difference was only seen between the HTTIPKV-liposome and no treatment groups, although the IP-injected mice continued to trend towards less growth.

We have previously shown that subcutaneous injection of BXPC3 and CAF cells produces tumors with a greater stromal content than $\mathrm{BXPC}_{3}$ injection alone (Figure 4.4). We performed HE staining of tumors from three mice per group in order to assess whether any changes in stromal content were apparent between treatment groups. Upon examination of three sections of three tumors from each group, no gross change in amount of stromal content was qualitatively observed (Figure $5.3 \mathrm{~b}$ ), suggesting that the decrease in tumor volume is not simply a reflection of a depletion of stroma.

Given the concern over the toxic off-target effects of systemically administered SB225002, we measured ex vivo amounts of liposomes in different organs (Figure 5.3 c). Liposomes accumulated in the blood-filtering organs (liver, kidney, and spleen), and had little or no accumulation in the lungs, heart, muscle, or bone. This suggests that the liposomes would be able to minimize the toxic effects experienced using systemic administration of CXCR2 inhibitors. We were able to detect both the targeted and nontargeted liposomes in the tumor tissue using IF because of the DiR that was incorporated into the lipid layer of the liposomes (Figure $5.3 \mathrm{~d}$ ). We co-stained with $\alpha$ SMA to indicate the location of CAFs and observed increased presence of the HTTIPKV-liposomes in the stromal fraction. 
a.
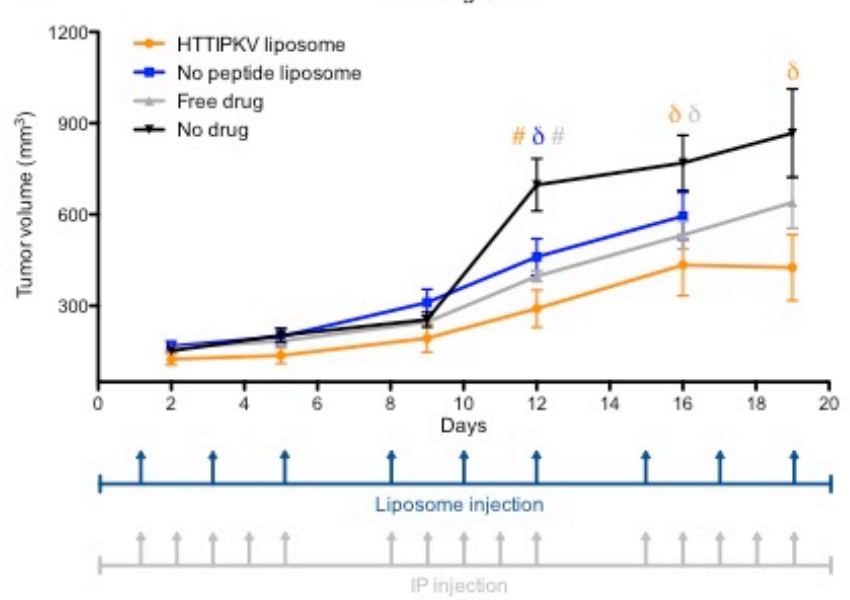

c.

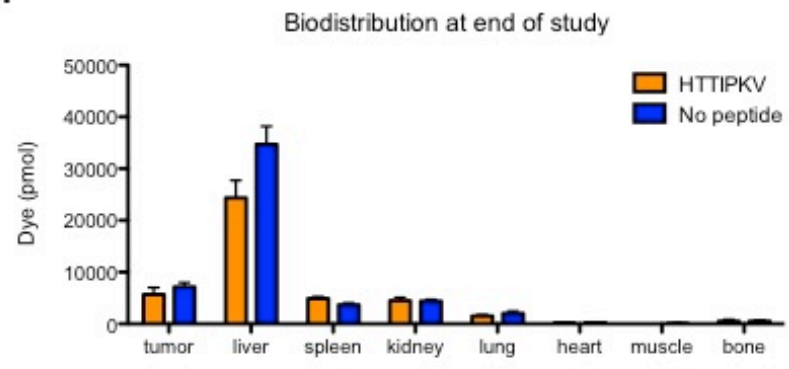

b.
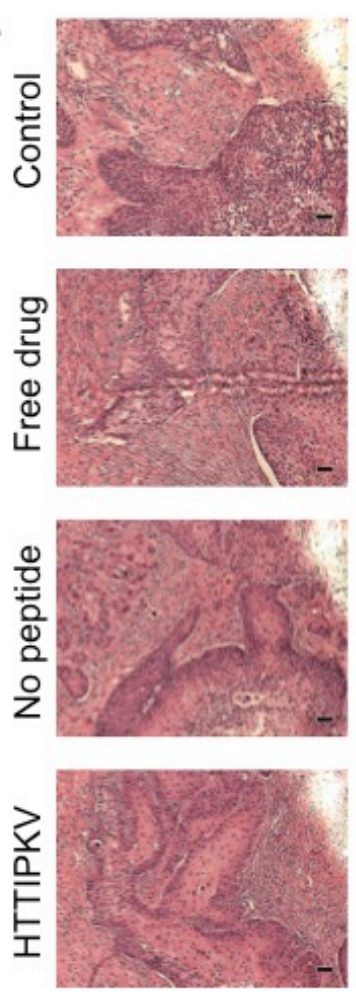

d.
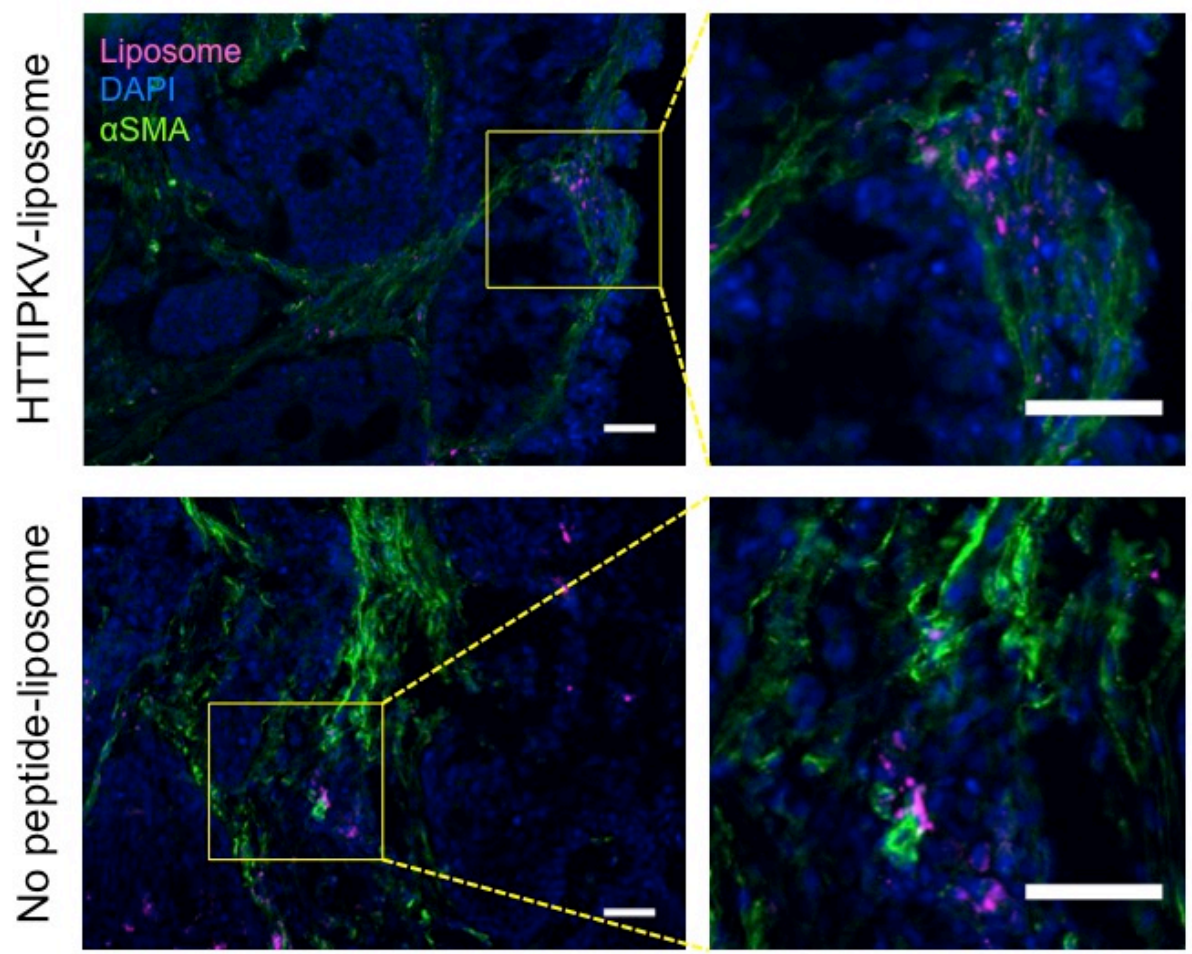

Figure 5.3: Subcutaneous tumors. (a) Tumor growth was measured via calipers for 19 days ( $n=10-12$ tumors/group). Dosage schedules for liposome and IP injection are shown below. (b) Representative images of HE staining of tumor sections from each treatment group show the stromal versus epithelial compartments. Scale bars, 50 um. (c) Ex vivo biodistribution was measured at the end of the study via the FMT. (d) IF images show the liposomes (DiR), nuclei (DAPI), and CAFs (aSMA). Scale bars, 50 um. 


\subsubsection{Blood pharmacokinetics of liposomes}

Blood pharmacokinetic measurements were taken for 24 hours after a single injection of liposomes using the heart blood pool as a surrogate for drawn blood as this is a more sensitive analysis using the FMT (Figure $5.4 \mathrm{a}$ and b) [87]. A two-compartment model where the first compartment (central) represents the well-perfused organs and blood and the second compartment (peripheral) represents the poorly-perfused tissues was fit to the time course data. For HTTIPKV-liposomes, the first and second phase coefficients were estimated as 0.0062 and 0.016 , respectively. The first and second phase coefficients for no peptide-liposomes were estimated as 0.024 and 0.042 , respectively.

a. Heart region-of-interest

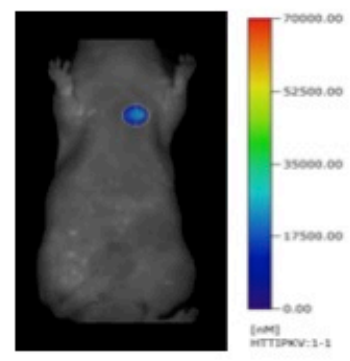

b.

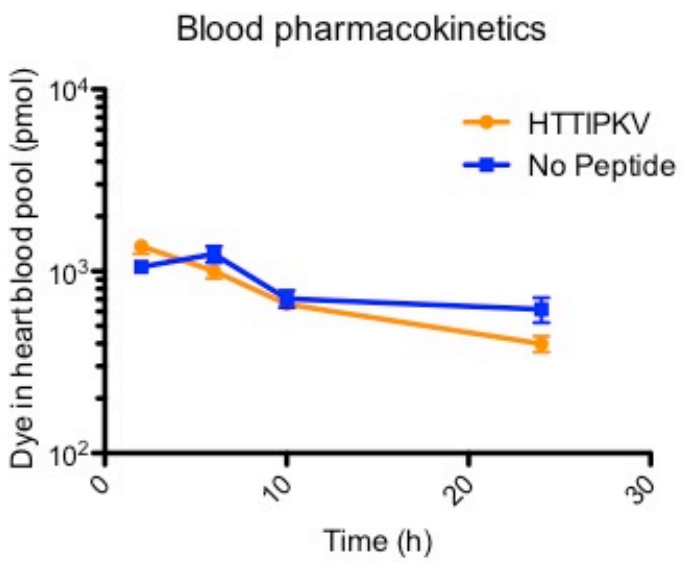

c. Two-compartment model
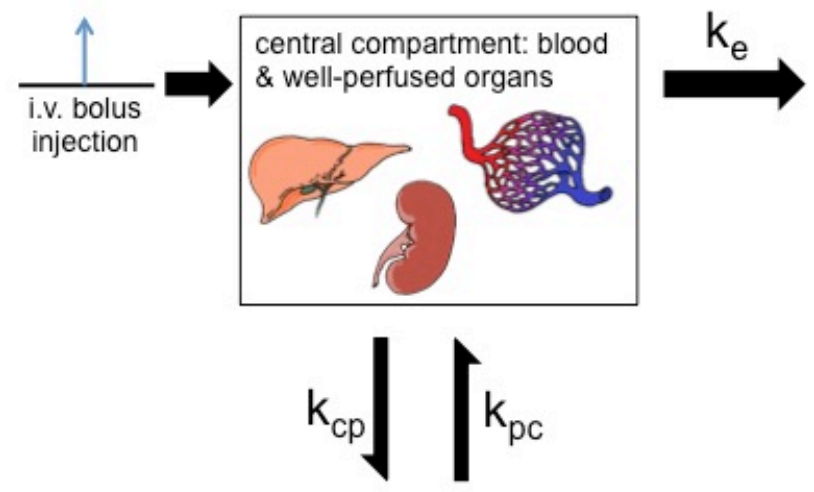

peripheral compartment: poorly-perfused tissues

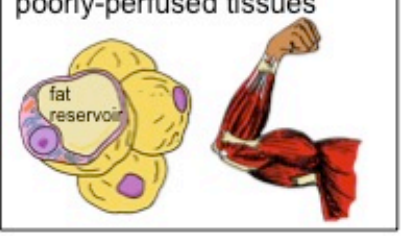

HTTIPKV: $\mathrm{C}_{\mathrm{p}}=0.15 \mathrm{e}^{0.0062 \mathrm{t}}+2.98 \mathrm{e}^{0.016 \mathrm{t}}$ No peptide: $\mathrm{C}_{\mathrm{p}}=0.25 \mathrm{e}^{0.024 t}+2.89 \mathrm{e}^{0.042 \mathrm{t}}$

Figure 5.4: Blood pharmacokinetics of liposomes. (a) An ROI was drawn around the heart blood pool and measured as a surrogate for a blood draw as shown in this representative FMT image. (b) The amount of dye in the heart ROI was measured for 24 hours after injection via FMT. (c) A two-compartment model was used to calculate the coefficients of the first and second phases of blood clearance for both types of liposome. Artwork courtesy of Travis Brinton. 
Since liposome pharmacokinetics are mostly defined by the liposome size, which was held constant between the two types of liposomes, the coefficients were similar. However, both the no peptide-liposome coefficients were greater, suggesting a higher retention and lower recirculation rate for the HTTIPKV-liposomes.

\subsubsection{Tumor section characteristics}

CXCR2 inhibition delivered via liposomes led to decrease in CTGF expression. CXC chemokines have been reported to induce CTGF expression and consequently cause tumor growth (figure 5.5 a) [11]. Therefore, we anticipated a decrease in CTGF expression for treatment groups. We homogenized sections of tumors from three mice of each group and ran a Western blot probing for CTGF and $\beta$-actin as a loading control (figure $5.5 \mathrm{~b}$ ). Quantitation of the blot via densitometry revealed a trend of decreasing

a.

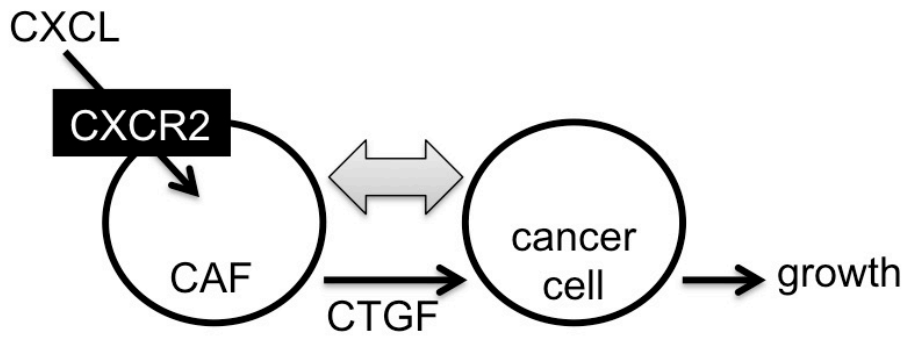

C.

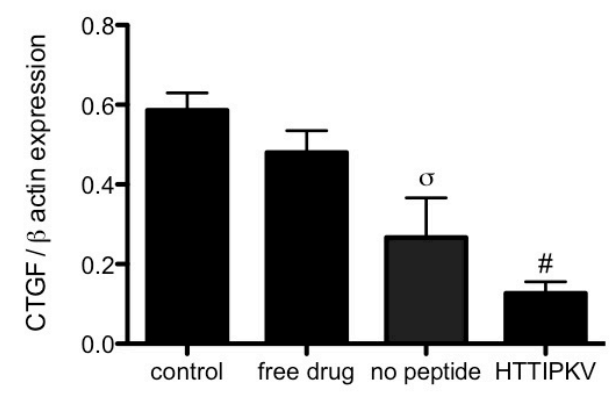

b.

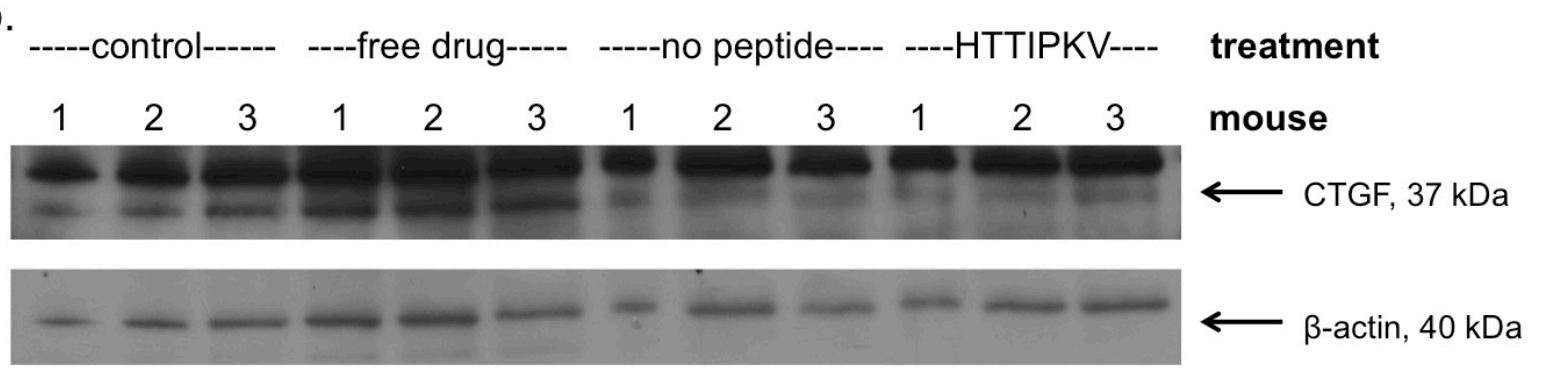

Figure 5.5: CTGF expression lowered in liposome treatment groups. (a) Proposed mechanism for increased growth due to CXCR2 inhibition. [11] (b) Western blot of homogenized tumor lysates from three mice per treatment group using anti-CTGF and antibeta actin (loading control). (c) Densitometry of CTGF western blot. 
CTGF expression for all treatment groups; the decrease was statistically significant for both of the liposomal treatment groups with the targeted liposome having the strongest effect (figure $5.5 \mathrm{c}$ ). Thus it is probable that the CXCR2 inhibitor is being effectively delivered by the HTTIPKV-liposomes and altering the CTGF expression.

Tumor vessel angiogenesis. Ijichi, et al. proposed that inhibition of angiogenesis contributed to the overall anti-tumor effect of SB225002. Therefore, we used CD31 staining to elucidate differences in angiogenesis caused by the different treatments. Average area fraction of vessels for no treatment, IP injection, no peptide-liposome, and

a.

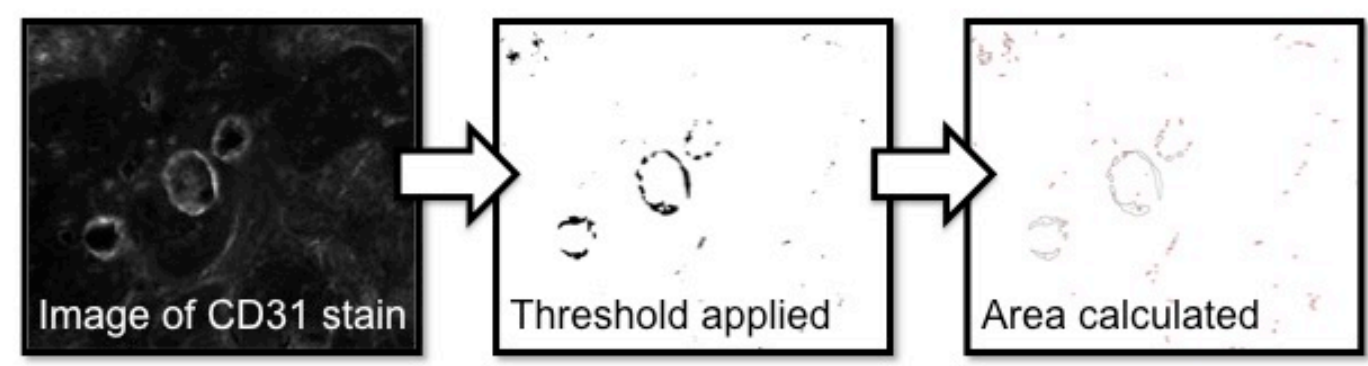

b.
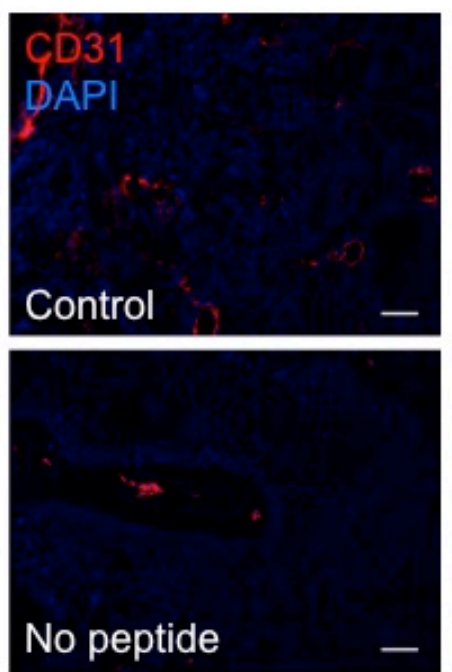
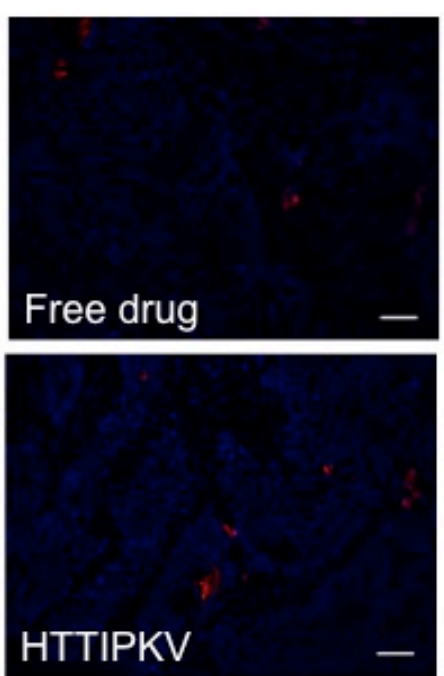

c.

CD31 quantification

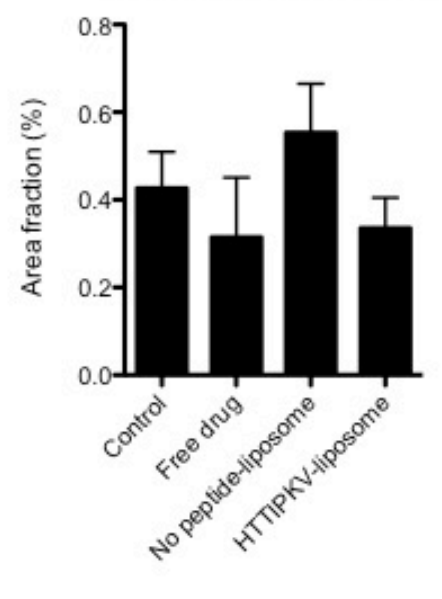

Figure 5.6: Tumor angiogenesis. (a) Images of sections stained for CD31 were analyzed with ImageJ by applying the same threshold to each image and then calculating the fraction of area occupied by the vessels. (b) Representative images of CD31 staining show the vessels in different treatment groups. Scale bar, 50 um. (c) Quantification of images was averaged across six images of three sections of three tumors (54 images total per treatment group). No statistical significance found with Student's t-test for $p<0.05$. 
HTTIPKV-liposome was measured using ImageJ (Figure 5.5 a) and found to be $0.43 \%$, $0.32 \%, 0.55 \%$, and $0.34 \%$, respectively, indicating a trend (though not statistically significant) of decreased angiogenesis for the IP-injected and HTTIPKV-liposome groups (Figure $5.5 \mathrm{~b}$ and $\mathrm{c}$ ).

Cell proliferation. We examined whether there was a difference in cell proliferation between treatment groups with IF of tumor sections using anti-Ki67 and quantified the using ImageJ (Figure $5.7 \mathrm{a}$ and b). Mean percent area fraction of cell proliferation for control, free drug, no peptide-liposome, and HTTIPKV-liposome treatment groups was $1.74 \%, 0.27 \%$, $0.90 \%$, and $0.37 \%$, respectively. Compared to the mice with no treatment,

a.

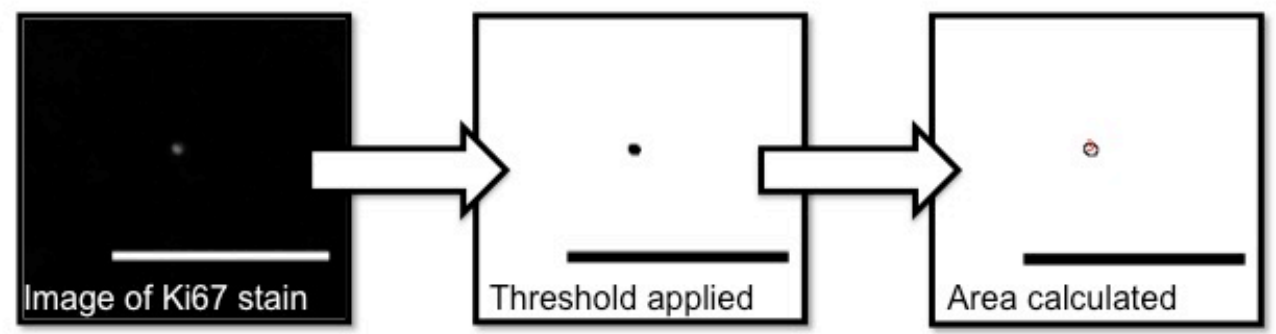

b.
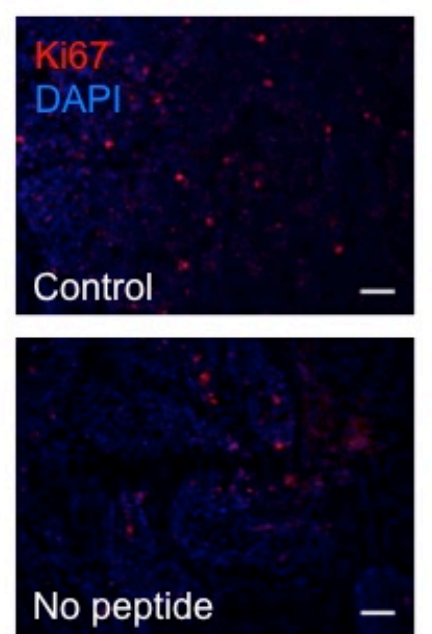
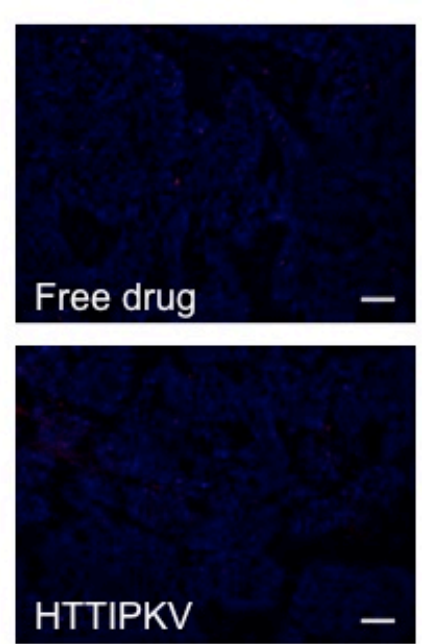

c.

Ki67 quantification

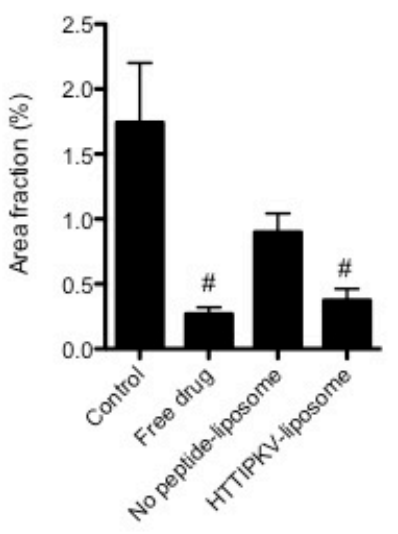

Figure 5.7: Cell proliferation. (a) Images of sections stained for Ki67 were analyzed with ImageJ (magnification of positive cell shown here) by applying the same threshold to each image and then calculating the fraction of area occupied by the stained area. Scale bar, 50 um.(b) Representative images of Ki67 staining show how the different treatment groups compare in cell proliferation. Scale bar, 50 um. (c) Quantification of images using five images of three sections of three tumors (45 images total per treatment group). \#p<0.01 statistical significance with Student's t-test. 
the mice that received HTTIPKV-liposome and those that received free drug had a statistically significant decrease in proliferation (Figure $5.7 \mathrm{c}$ ). The mice with no peptideliposome treatment also had a lower mean cell proliferation, but this trend did not have statistical significance.

\subsection{Discussion}

The value of incorporating drugs into liposomes is epitomized by the case of doxorubicin. Doxorubicin is a drug that is used to treat a variety of cancers because it intercalates DNA. However, it can also cause a life-threatening cardiotoxicity that limited its clinical use. Encapsulation of doxorubicin in a liposome (known as Doxil) decreased the cardiotoxicity and allowed a larger cumulative dose to be given to patients, significantly improving doxorubicin's therapeutic index [88]. Similarly, the use of liposomes to deliver other drugs could reduce toxicity issues and result in improved drug distribution. Two critical attributes of liposomes contribute to their success and guided our liposome design: size and composition.

Control over the size of liposomes allows them to preferentially locate in tumors due to the EPR effect. Although tumor vessels contain the same constitutive components (pericytes, endothelial cells, and basement membrane) as normal vessels, the endothelial cells of tumor vessels are unusual in that they do not form a monolayer, are disorganized, have an irregular shape, and lack normal barrier function [89]. A size range of $100-200 \mathrm{~nm}$ is large enough to avoid extravasation across healthy vessels, but small enough to fit through larger tumor vessel pores $(<2 u m)$ [88]. In addition to taking advantage of the EPR effect, it is important to take into account how the liposomes are cleared in order to increase circulation time.

The optimal size range of $100-200 \mathrm{~nm}$ for extravasation across tumor vessels is already large enough to minimize uptake in the kidneys, which filter out particles $<5-6 \mathrm{~nm}$. 
However, the liver tends to uptake particles $<50 \mathrm{~nm}$ or $>100 \mathrm{~nm}$ and the larger the size after $100 \mathrm{~nm}$, the more the spleen traps [90]. Thus, we selected to size our liposomes slightly larger than $100 \mathrm{~nm}$ as it is an optimal size for the EPR effect and longest circulation time. While it is important to increase circulation time to deliver as much drug as possible to the tumor site, it is also necessary that the body be capable of eventually clearing the liposomes from the body. As is typical of liposomes, we noted considerable uptake of our liposomes in the liver and spleen (Figure $5.1 \mathrm{~g}$ ), suggesting that these are their main clearance routes.

The next essential characteristic of liposomes is composition. Our liposomes are made of DOPC, DSPE, and cholesterol. These components are biocompatible because they are dietary lipids and present in the cellular membrane [88]. Our lipid:cholesterol ratio provided bilayer integrity to prevent drug from leaking in circulation. The DSPE provided an anchor for PEG, which prevents the reticuloendothelial system (RES) from sequestering the liposomes. The DSPE was also the site of attachment of our targeting peptides. Due to the EPR effect, non-targeted liposomes will preferentially accumulate in tumors. However, the targeting peptides acted to increase the retention of our liposomes in the tumor area (Figure $5.1 \mathrm{c}$ ) in addition to binding to the surface of the CAFs so that the liposomes degrade and deliver their payload close to the CAFs.

\subsection{Methods}

\section{Liposome formulation}

Peptides were synthesized at Tufts University Peptide Synthesis Core Facility as [H]-HT-T-I-P-K-V-G-G-S-K(fitc)-G-C-[NH2] and [H]-A-P-P-I-M-S-V-G-G-S-K(fitc)-G-C[NH2] using standard FMOC chemistry and Rink-Amide resin. Purified product was used for all experiments. 
$4 \mathrm{mg}$ of FITC-labeled peptide was dissolved in $900 \mathrm{uL}$ of degassed PBS/1 mM EDTA and $9 \mathrm{mg}$ of 1,2-distearoyl-sn-glycero-3-phosphoethanolamine-N-[methoxy(polyethylene glycol)-3400]-maleimide (DSPE-PEG $3400-$ maleimide, Avanti polar lipids) dissolved in $100 \mathrm{uL}$ methanol. The two solutions were combined while bubbling with argon gas and then freeze-dried.

20.5 mg 1,2-Dioleoyl-sn-glycerol-3-phosphocholine (DOPC, Avanti polar lipids), $9.5 \mathrm{mg}$ 1,2-disteroyl-sn-glycerol-3-phosphocholine cholesterol (DSPC-cholesterol, Avanti polar lipids), $9.5 \mathrm{mg}$ DSPE-PEG ${ }_{2000}$ (Avanti polar lipids), $1 \mathrm{mg}$ freeze dried DSPE-PEG ${ }_{3400^{-}}$ maleimide-conjugated peptide, and $0.5 \mathrm{mg}$ DiR (Invitrogen) in methanol $(25 \mathrm{mg} / \mathrm{mL}$ ) were dissolved in $2 \mathrm{~mL}$ chloroform (Sigma-Aldrich). After evaporation, the lipid layer was hydrated by adding $2 \mathrm{~mL}$ saline, and subjected to three freeze-thaw cycles. The liposomes were sized by passing the solution 41 times through a manual extruder with a $200 \mathrm{~nm}$ filter.

\section{Nanosight analysis of liposomes}

The size and concentration of the liposomes was determined using a NanoSight NS30o (Malvern Instruments), using the NanoSight NTA3.o software. Liposomes were diluted in saline and then run through the following script: set temperature to $25^{\circ} \mathrm{C}$, delay for 5 seconds, capture for 30 seconds, syringe in more sample, delay for 5 seconds, repeat four times, turn off the laser. The screen gain was set to 1.0 and the camera level to 10. Videos captured of the samples were processed using a screen gain of 7.0 and a detection threshold of 3 , then exported.

\section{In vivo liposome-based validation}

Mice were tumored as described above (see In vivo phage validation). Liposomes were then injected (50,000 pmol DiR) into the tail vein of the mice and tumor accumulation 
was tracked using the FMT over a two-week time course. Three groups of liposomes were tested: HTTIPKV-conjugated, APPIMSV-conjugated, and no peptide. Each group was composed of 10-12 tumors. Total pmol of dye measured in the tumor area was determined by drawing a region of interest around the tumors as visualized on the reflectance images. At $24 \mathrm{~h}$, two mice were sacrificed. Their organs and tumors were excised and measured with the FMT to determine the biodistribution.

\section{Pharmacokinetic analysis}

In order to generate the coefficients of accumulation and clearance for tissue (tumor) accumulation, we took the log of our data from our two-week time course (Figure $5.1 \mathrm{c}$ ) and performed linear regression using Excel. We then assessed our fit in MATLAB by generating curves of our fit using these coefficients and comparing them to the data from the experiment (Figure 5.1 d). To assess the clearance from the blood in our SB225002 study, we estimated the change in amount of dye in the blood using FMT images of the heart (Figure 5.4 a) [87]. We generated curves with the log of this data and again used linear regression to estimate the coefficients associated with the first and second phase blood clearance.

\section{SB225002-loading of liposomes}

Liposomes were produced in the same way as described above, but instead of hydrating the lipid membrane in saline, they were hydrated in an SB225002-containing solution (0.5 mg / mL).

\section{Tumor section staining.}

Tumors sections were prepared for IHC and IF as described in chapter four methods and stained with anti-CTGF (Abcam, 1:200, 20 minutes) then anti-rabbit secondary (1:250, 20 minutes), anti-CD31 (BD biosciences, 1:100, 20 minutes) then anti-rat secondary 
(1:250, 20 minutes), or anti-Ki67 (abcam, 1:250, 20 minutes) then anti-rabbit secondary (1:250, 20 minutes). Images were imported into ImageJ and converted to 8-bit. After applying the threshold, the area fraction was measured for each image. Section averages were entered into Prism to find overall means for each treatment group and tested for statistical significance between groups using the Student's t-test. 


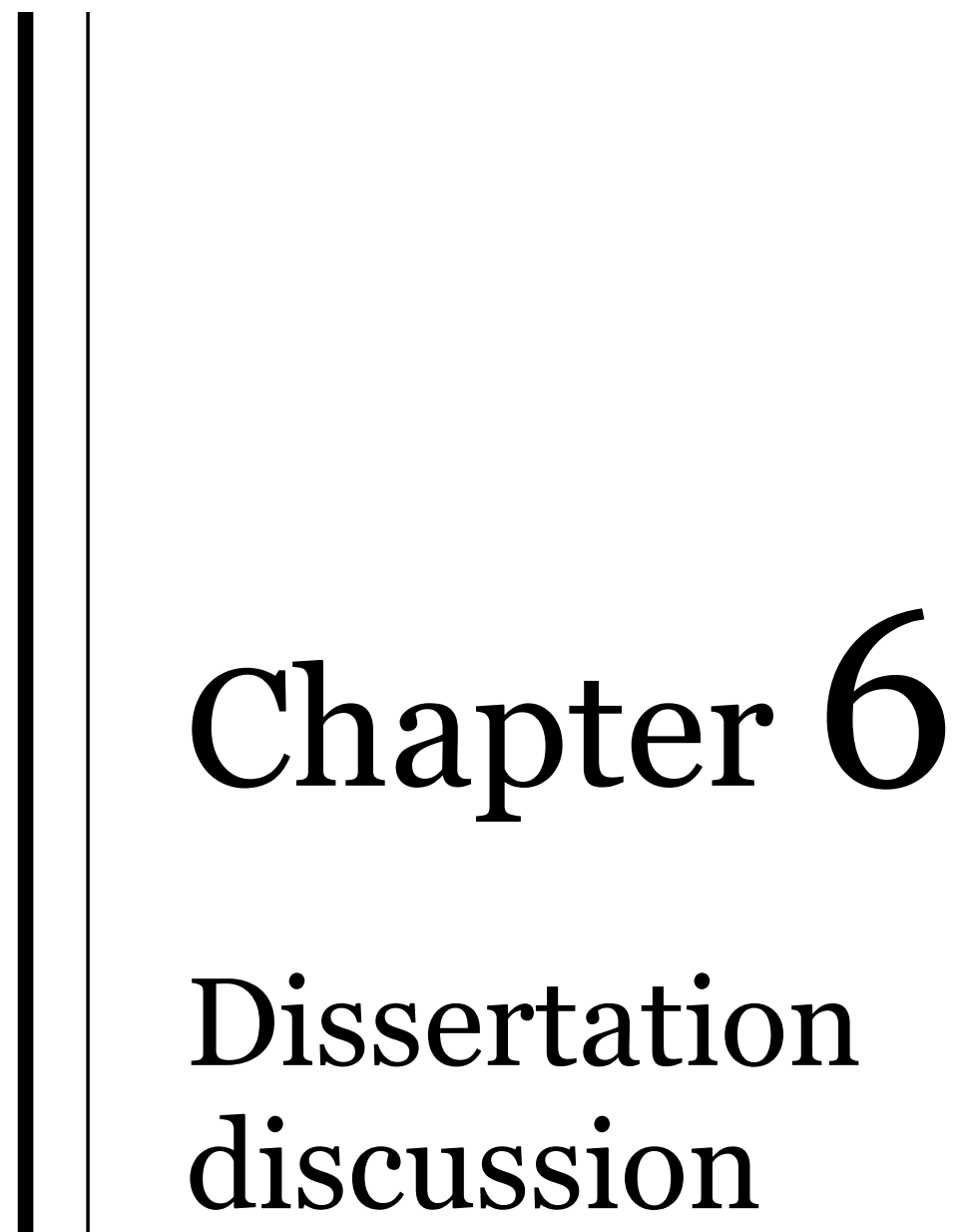




\subsection{Developing a targeted, stromal therapy}

\subsubsection{Historical context of our research}

Prior to the turn of the $20^{\text {th }}$ century, many were skeptical that drugs could ever cure advanced cancers. Some hope was garnered from the success of drugs that resulted from World War II-related programs that accidentally discovered a chemotherapeutic use for
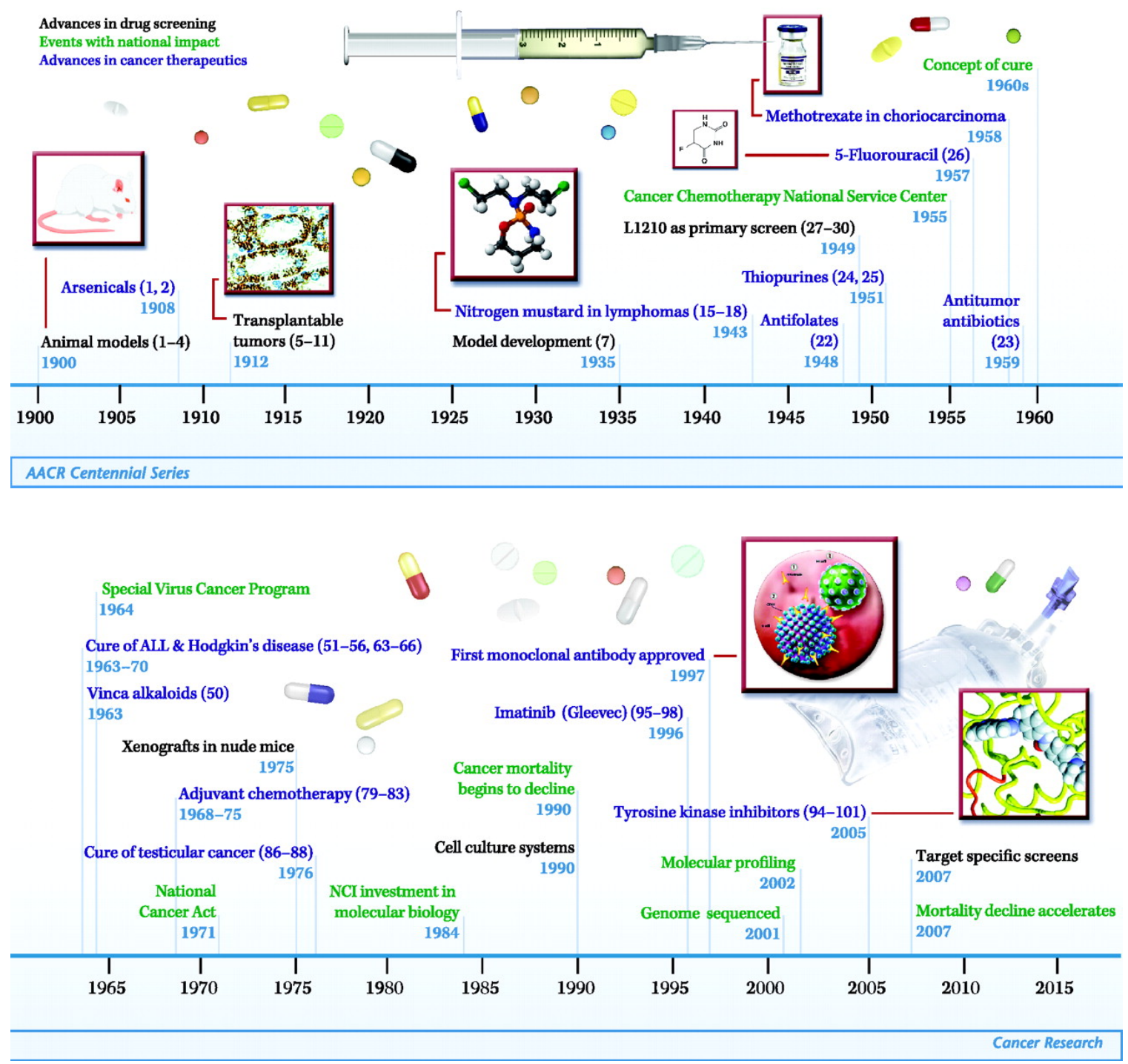

Figure 6.1 Evolution of cancer chemotherapy. Key historical advances in the search for effective cancer treatments. Parenthetical citations refer to references from the original article.

Reprinted from [91], "A history of cancer chemotherapy", 68/21, VT DeVita Jr. and E Chu, 8643-53, copyright (2008), with permission from AACR. 
chemical warfare agents. However, it was not until later that real breakthroughs shifted opinions: combination chemotherapy effectively cured acute childhood leukemia (1960’s) and advanced Hodgkin's disease (1970's). It was a natural next step to combine chemotherapy with surgery and/or radiation and this became the new clinical standard [91]. While chemotherapy became a valuable tool in treating cancer, it was plagued with toxic effects. Siddhartha Mukherjee described toxic effects of a new chemotherapy drug, Cisplatin, during this era as, "an unremitting nausea, a queasiness of such penetrating force and quality that had rarely been encountered in the history of medicine: on average, patients treated with the drug vomited twelve times a day...[Cispatin was] the quintessential example of how curing cancer involved pushing patients nearly to the brink of death" [92]. Today, toxicity is still one of the most prominent issues in cancer treatment. This explains the emphasis now placed on molecularly-targeted therapies, beginning with use of inhibitors, like imatinib, which targets the Bcr-Abl tyrosine kinase (1996) [91] and including also the idea that any drug can be delivered using drug delivery vehicles. Indeed, the revolutionary ability of drug delivery vehicles to overcome toxic side effects is highlighted by the success of encapsulating doxorubicin into a liposome (Doxil, 1995) [93]. This dissertation work responds to a clinical need that has arisen as a result of the movement towards targeted therapy, namely, a methodology to rapidly develop targeted moieties and nanoparticles and presents peptides to a novel site, the CAFs.

In the years immediately following the introduction of Doxil, the use of liposomes to alter the toxicity profile of drugs has increased with the FDA approving Daunoxome (Daunorubicin, 1996), Ambisome (Amphotericin B, 1997), and Depocyte (Cytarabine, 1999). Just this year, Onivyde (Irinotecan, 2015) was approved to be used in combination with 5 -FU and Leucovorin to treat advanced PDAC in patients that have previously tried GEM. In a randomized trial, patients that received the Onivyde/5- 
FU/Leucovorin combination therapy survived 6.1 months compared to the 4.2 months for patients receiving 5-FU/Leucovorin [94]. The results achieved with these drugs paves the way for FDA approval of more liposomal drugs and the case of Onivyde stresses the potential for combination therapy. In the randomized trial mentioned above, patients who received only Onivyde had no survival improvement compared to the 5FU/Leucovorin group [94].

The research presented in this dissertation fits well with current trends in cancer therapy towards targeted liposomal formulations and exploiting additive or synergistic drug effects through the use of combination therapy while also furthering the field two important ways. Firstly, our liposome uses a targeting moiety to further specify where a drug is delivered, whereas the current FDA-approved liposomes rely entirely on the EPR effect. In addition to locating the liposomes next to a specific cell type, the targeting moiety is also capable of increasing retention in that area by decreasing recirculation rates. In our mouse studies with dye-incorporated liposomes, the targeting moiety increased the AUC as compared to non-targeted liposomes and in our SB225002 pilot study, we saw that the addition of the targeting moiety enhanced the results of the treatment with trends towards less CTGF expression, decreased angiogenesis, and lower rates of cell proliferation. Secondly, we have developed a targeted liposome to the PDAC CAFs, not the tumor epithelial cells. To our knowledge, these are the first CAF-targeted peptides to be presented for PDAC. Targeted to stromal cells, our liposomes are geared towards combatting the tumor-stromal interactions and it is highly likely that synergism would occur with epithelial-directed drugs.

\subsubsection{Influence of an evolving biological understanding of PDAC}

Cancer therapy has been limited by scientific understanding of the nature of cancer. Slow research progression can occur by trial and error and even based on false assumptions. 
Sidney Farber, deemed the "father of modern chemotherapy" only succeeded in treating leukemia with antifolates after observing a decrease in survival of children to whom he had given folates [95]. The converse situation is therapeutic research inspired by enhanced understanding of underlying biological principles. In the case of PDAC, an evolving knowledge of the drivers of the disease can and should guide innovation in therapy.

Understanding of PDAC has affected the research direction of this dissertation work. PDAC characteristics include an extensive desmoplastic reaction, hypovascularity, hypoxia, evasion of the host immune system, early metastasis, and chemoresistance. The observation that PDAC has such a large stromal component begs the questions why and how is it related to the other observed characteristics? These questions have been at the center of recent research that has shown a more prominent role of the microenvironment than was once assumed. PDAC is now thought to act like an organ system and is described as having two compartments, stromal and epithelial, that communicate back and forth and together influence the disease progression. In fact, a number of lethal characteristics of PDAC mentioned above including evasion of the host immune system, early metastasis, and chemoresistance-have now also been found to be a partially a product of the influence of the stromal compartment [96]. For this reason, we selected to target a principle component of the stromal compartment, CAFs, in this dissertation research. Furthermore, this target selection mitigates delivery issues that often occur with other drugs due to another PDAC characteristic, hypovascularity. A lack of blood vessels makes the epithelial component of the tumor is difficult to reach, but the CAFs are located in closer proximity to blood vessels, in part because of their role in stimulating angiogenesis. Even the PDAC characteristic of hypoxia is essential to our dissertation research design as it is this harsh environment that leads to the decreased 
integrity of the membrane of our liposomes and allows release of the drug trapped inside.

\subsection{Dissertation research conclusions}

We have built on phage display technology and deep sequencing capabilities to develop a pipeline for targeted nanoparticle development. Within this framework, targeted moieties can quickly be identified that bind disease specific proteins, cells, or tissue. The quantitative analysis that we provide in the form of a user-friendly MATLAB-based program, NGSanalyze, increases predictive power and facilitates selection of ideal targeted peptide candidates. The methodology we put forth to tether these peptides to biocompatible liposomal drug delivery vehicles makes it possible to go from target of interest to targeted moiety to targeted drug delivery in the short timeframe of one to two months.

Since our methodology finds targets to any cell type of interest in a short timeframe, it can be applied to patient-derived samples and therefore is potentially applicable in personalized medicine to generate patient-tailored drug delivery systems. As previously mentioned, targeted ligands have numerous applications in an experimental, therapeutic, and imaging setting. Development of the matrix analysis allows facile scanning of peptides across multiple cell types with which the drug vehicle would be in contact physiologically and sets apart our approach from other applications of NGS to phage display. While NGS has been used to increase the depth of screening results, our in silico analysis delivers high predictability of successful peptide sequences thus decreasing amount of time for validation. In fact, our methodology streamlines the entire process from ideal cell target to nanoparticle, making targeted ligand discovery and subsequent drug delivery or imaging available to scientists from diverse areas of research, without necessitating extensive training. 
Liposomal formulations of drugs are obtaining FDA-approval and increasing in popularity. In this dissertation, we show an effective CAF-targeted liposome. We have validated the targeted capability of two peptides using both in vitro and in vivo studies with the peptide displayed by bacteriophage. We have further shown that these targeted peptides can increase accumulation of liposomes in subcutaneous pancreatic tumors on mice $2.25^{-}$and $1.75^{-f o l d}$ over non-targeted nanoparticles. In assessing the biodistribution of our targeted liposomes, there is marked accumulation only in the tumor and blood-filtering organs (liver, spleen, and kidneys) suggesting that our CAFtargeted liposomes have great potential in overcoming drug toxicity issues.

We have demonstrated the drug-carrying capacity of the CAF-targeted liposomes in a pilot study using SB225002, a CXCR2 inhibitor and examined the associated blood pharmacokinetics. CXCR2 inhibition has been shown to be an effective treatment in PDAC with the risk of severe toxic side effects [11]. Our CAF-targeted liposomes were able to produce the anti-tumor effects of seen with intraperitoneal administration of the free drug form when we injected them in mice with subcutaneous pancreatic tumors. We observed a trend in decreased angiogenesis and CTGF expression and statistically significant decrease in cell proliferation compared to mice that received no treatment. Quantified IF images of cell proliferation marker Ki67 revealed mean percent area fraction for control, free drug, no peptide-liposome, and HTTIPKV-liposome treatment groups to be $1.74 \%, 0.27 \%, 0.90 \%$, and $0.37 \%$, respectively. This pilot study of SB225002-loaded, CAF-targeted liposomes marks the potential to circumvent toxicity issues associated with systemic use of CXCR2 inhibitors.

In conclusion, studies in this dissertation have led to a new approach to targeted peptide discovery and nanoparticle development as well as demonstrated the power of this process by producing CAF-targeted liposomes that succeeded in delivering a CXCR2 
inhibitor in a pilot study. Thus we anticipate that this process and the included MATLAB program will prove beneficially to diverse areas of research and possibly a useful tool in the advent of personalized medicine. The CAF-targeted peptides stand to have important consequences in PDAC therapy and have the potential to be developed into an imaging agent for early detection as well as move forward the biological understanding of PDAC through identification of binding partners.

\subsection{Future directions}

\subsubsection{Imaging agent for early detection}

The propensity of PDAC to metastasize at early stages greatly complicates treatment of the disease. Prior to advancement to metastatic disease, surgical resection can effectively cure PDAC. However, in order to treat pre-metastatic PDAC, it must be detected when the tumor diameter is less than one centimeter, ideally when it is still a precursor lesion that will progress to malignancy [1]. With the current tools available, attempts to image the tumor epithelial cells have not yet been able to consistently achieve detection [97]. In contrast to the tumor epithelial cells, the stromal CAFs are closer to the blood vessels and 10-100 times more abundant [33]. Thus it may actually be easier to detect early PDAC or its precursor lesions via imaging the CAFs rather than the tumor epithelial cells. The peptides that we have discovered as part of this dissertation work bind to the CAFs and could be used to image them. In order to use the CAF-targeted peptides in this way, further experimentation is necessary. The peptides must be radiolabeled and studies carried out to optimize and determine how sensitive this method of detection is. The Kelly lab has submitted a grant proposing to do this work and develop a PET agent with Gallium-68-labeled CAF-targeted peptides. 


\subsubsection{Binding partner identification}

The Kelly lab has been successful in identifying the binding partners of certain peptides using pulldown assays in which we first biotinylate the phage and add a photolyzable crosslinker, allow them to bind to the cells, use UV light to cross link the phage to its binding partner, lyse the cells, and use streptavidin beads to pull out the phage-protein

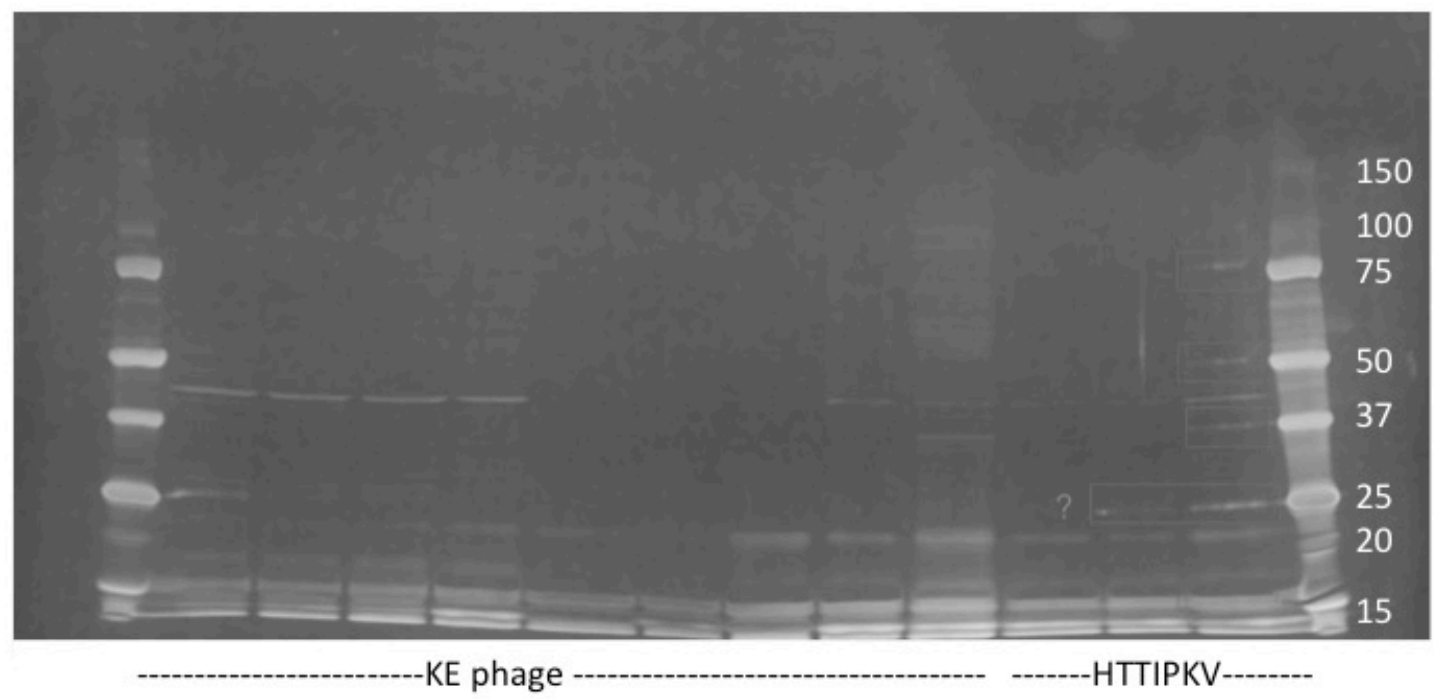

Figure 6.2 Silver stain from pulldown assay. This silver-stained gel shows the bands that are detected from multiple pulldown assays of KE phage and HTTIPKV phage. Bands thought to potentially be unique are boxed.

complex [39]. By running these proteins on a gel and using silver stain, we can compare binding a targeted peptide to that of KE (no displayed peptide) phage. A band unique to the targeted phage represents its binding partner and can be identified by mass spectrometry. However, in the case of other peptides, including the CAF-targeted peptides presented in this dissertation, our attempts to identify the binding partners have been thwarted by non-specific phage binding, which causes a large number of proteins to be complexed with the phage and complicates subsequent analysis (Figure 6.2). Therefore, we are building on our previous efforts [39] by including stable isotope labeling by amino acids in cell culture (SILAC) technology. The idea is that by culturing cells with "light" or "heavy" amino acids, we will be able to quantitatively distinguish 
protein levels between $\mathrm{KE}$ and targeted phage via mass spectrometry [40]. We are optimizing this process plan to use it to identify the binding partners of the CAF-targeted peptides. Not only will this enable us to use antibody-staining of panels of human tissues to ascertain where and how much of this protein is expressed throughout the body, but these binding partners may lend important biological insight into the role of CAFs in PDAC and lead to further experiments to understand their functions.

\subsubsection{Sophisticated CXCR2 studies}

The positive results from the pilot study of SB225002 in this dissertation research warrant further, more extensive experiments. We suggest that these studies first take on the following: (1) remote loading, (2) drug dose escalation, and (3) toxicity. Firstly, we used the hydration method to load SB225002 into our liposomes. While this method is an acceptable and fast means of discerning the potential of loaded-liposomes, more efficient loading can be achieved using the remote loading method. In the hydration method, dried lipid layers are simply exposed to a solution containing the drug and as they hydrate and form liposomes the drug solution is trapped inside. By contrast, in remote loading, the lipids are sonicated with an ammonium sulfate solution so that they form around ammonimum sulfate droplets, which are then replaced by the drug during incubation at 50 degrees Celsius. High performance liquid chromatography (HPLC) can subsequently be used to determine the amount loaded and release profile.

Secondly, we recommend drug dose escalation studies. We completed studies by comparing equal weekly doses of free and liposome-incorporated SB225002. However, the AUC from our mouse time course data (Figure $5.1 \mathrm{e}$ ) is evidence that the targeted liposomes are capable of more efficient drug delivery. Therefore, studies could be designed to determine by how much the amount of drug could be reduced and still be effective. 
Thirdly, we suggest formal toxicity studies. Our biodistribution data (Figure $5.3 \mathrm{c}$ ) indicates that the toxicity issues surrounding SB225002 may be minimized by using our CAF-targeted liposomes. In our studies, we employed subcutaneous mouse models using human cells, which necessitates the use of mice with a genetic mutation that causes a deteriorated or absent thymus. Investigation of the immune response, in particular difference in neutropenia induction, resulting from our CAF-targeted delivery versus free drug administration would be better carried out in animals with an intact immune system. This and further studies of how the toxicity profile differs between the two routes of administration would be important in considering the best clinical avenue. We feel that these three experimental directions would be the best first step in furthering this line of research. 


\section{References}

1. Li D, Xie K, Wolff R, Abbruzzese JL. Pancreatic cancer. Lancet 363(9414), 1049-57 (2004).

2. Li Q, Yuan Z, Yan H, Wen Z, Zhang R, Cao B. Comparison of Gemcitabine combined with targeted agent therapy versus Gemcitabine monotherapy in the management of advanced pancreatic cancer. Clin Ther Epub ahead of print. (2014).

3. Tsunoda T, Yamamoto Y, Kimoto M, Imai H, Iwamoto S, Kawasaki S, Kawashima K, Tadaoka Y, Majima T, Onuma E, Iki K, Kubozoe T, Eto T. Staging and treatment for patients with pancreatic cancer. How small is an early pancreatic cancer? $J$ Hepatobillary Pancreat Surg 5(2), 128-32 (1998).

4. Wilkowski R, Wolf M, Heinemann V. Primary advanced unresectable pancreatic cancer. Recent Results Cancer Research 177:79-93 (2008).

5. Kim MP, Gallick GE. Gemcitabine resistance in pancreatic cancer: picking the key players. Clin Cancer Res 14(5), 1284 (2008).

6. Burris III HA, Moore MJ, Andersen J, Green MR, Rothenberg ML, Modiano MR, Cripps MC, Portenoy RK, Storniolo AM, Tarassoff P, Nelson R, Dorr FA, Stephens CD, Von Hoff DD. Improvements in survival and clinical benefit with gemcitabine as first-line therapy for patients with advanced pancreas cancer: a randomized trial. J Clin Oncol 15(6), 2403-13 (1997). 
7. Hwang RF, Moore T, Arumugam T, Ramachandran V, Amos KD, Rivera A, Ji B, Evans DB, Logsdon CD. Cancer-Associated Stromal Fibroblasts Promote Pancreatic Tumor Progression. Cancer Res 68(3), 918-26 (2008).

8. Özdemir BC, Pentcheva-Hoang T, Carstens JL, Zheng X, Wu CC, Simpson TR, Laklai H, Sugimoto H, Kahlert C, Novitskiy SV, De Jesus-Acosta A, Sharma P, Heidari P, Mahmood U, Chin L, Moses HL, Weaver VM, Maitra A, Allison JP, LeBleu VS, Kalluri R. Depletion of carcinoma-associated fibroblasts and fibrosis induces immunosuppression and accelerates pancreas cancer with reduced survival. Cancer Cell 25(6), 719-34 (2014).

9. Rhim AD, Oberstein PE, Thomas DH, Mirek ET, Palermo CF, Sastra SA, Dekleva EN, Saunders T, Becerra CP, Tattersall IW, Westphalen B, Kitajewski J, FernandezBarrena MG, Fernandez-Zapico ME, Iacobuzio-Donahue C, Olive KP, Stanger BZ. Stromal elements act to restrain, rather than support, Pancreatic Ductal Adenocarcinoma. Cancer Cell 25(6), 735-47 (2014).

10. Dimou A, Syrigos KN, Saif MW. Overcoming the stromal barrier: technologies to optimize drug delivery in pancreatic cancer. Ther Adv Med Oncol 4(5), 271-9 (2012).

11. Ijichi H, Chytil A, Gorska AE, Aakre ME, Bierie B, Tada M, Mohri D, Miyabayashi K, Asaoka Y, Maeda S, Ikenoue T, Tateishi K, Wright CVE, Koike K, Omata M, Moses HL. Inhibiting Cxcr2 disrupts tumor-stromal interactions and improves survival in a mouse model of pancreatic ductal adenocarcinoma. J Clin Invest 121(10), 4106-17 (2011).

12. Campbell LM, Maxwell PJ, Waugh DJ. Rationale and means to target proinflammatory Interleukin-8 (CXCL8) signaling in cancer. Pharmaceuticals (Basel) 6(8), 929-59 (2013). 
13. Hoyert DL, Xu J. Deaths: preliminary data for 2011. Natl Vital Stat Rep 61(6), 1-51 (2012).

14. Heron M. Deaths: leading causes for 2009. Natl Vital Stat Rep 61(7), 1-94 (2012).

15. Neessee A, Krug S, Gress TM, Tuveson DA, Michl P. Emerging concepts in pancreatic cancer medicine: targeting the tumor stroma. Onco Targets Ther 7, 3343 (2013).

16. Fukushige S, Horil A. Road to early detection of pancreatic cancer: attempts to utilize epigenetic biomarkers. Cancer Lett 342(2), 231-7 (2014).

17. Hazel AF, Kimmelman AC, Stanger BZ, Bardeesy N, Depinho RA. Genetics and biology of pancreatic ductal adenocarcinoma. Genes Dev. 2o, 1218-49 (2006).

18. Hruban RH, Maitra A, Goggins M. Update on pancreatic intraepithelial neoplasia. Int J Clin Exp Pathol 1, 306-316 (2008).

19. Hruban RH, Goggins M, Parsons J, Kern SE. Progression model for pancreatic cancer. Clin Cancer Res 6, 2969-72 (2000).

20. Klapman J, Malafa MP. Early detection of pancreatic cancer: why, who, and how to screen. Cancer Control 15, 280-7 (2008).

21. SEER stat fact sheets: prostate cancer, published by National Cancer Institute (2005-2011), available: seer.cancer.gov.

22. SEER stat fact sheets: female breast cancer, published by National Cancer Institute (2005-2011), available: seer.cancer.gov.

23. Cascinu S, Falconi M, Valentini V, Jelic S, EMSO guidelines working group. Pancreatic cancer: EMSO clinical practice guidelines for diagnosis, treatment and follow-up. Ann Oncol 21 (2010). 
24. Burris HA $3^{\text {rd }}$, Moore MJ, Andersen J, Green MR, Rothenberg ML, Modiano MR, Cripps MC, Portenoy RK, Storniolo AM, Tarassoff P, Nelson R, Dorr FA, Stephens CD, Von Hoff DD. J Clin Oncol 15, 2403-13 (1997).

25. Dahan L, Bonnetain F, Ychou M, Mitry E, Gasmi M, Raoul JL, Cattan S, Phelip JM, Hammel P, Chauffert B, Michel P, Legoux JL, Rougier P, Bedenne L, Seitz JF, Federation Francophone de Cancerologie Digestive. Combination 5-flurouracil, folinic acid and cisplatin ( $\mathrm{LV}_{5} \mathrm{FU}_{2}-\mathrm{CDDP}$ ) followed by gemcitabine or the reverse sequence in metastatic pancreatic cancer: final results of a randomised strategic phase III trial (FFCD 0301). Gut 59, 1527-34 (2010).

26. Abou-Alfa GK, Letourneau R, Harker G, Modiano M, Hurwitz H, Tchekmedyian NS, Feit K, Ackerman J, De Jager RL, Eckhardt SG, O’Reilly EM. Randomized phase III study of exatecan and gemcitabine compared with gemcitabine alone in untreated advanced pancreatic cancer. J Clin Oncol 24, 4441-7 (2006).

27. Kindler HL, Ioka T, Richel DJ, Bennouna J, Letourneau R, Okusaka T, Funakoshi A, Furuse J, Park YS, Ohkawa S, Springett GM, Wasan HS, Trask PC, Bycott P, Ricart AD, Kim S, Van Cutsem E. Axitinib plus gemcitabine versus placebo plus gemcitabine in patients with advanced pancreatic adenocarcinoma: a double-blind randomised phase 3 study. Lancet Oncol 12, 256-62 (2011).

28. Colucci G, Labianca R, Di Costanzo F, Gebbia V, Carteni G, Massidda B, Dapretto E, Manzione L, Piazza E, Sannicolo M, Ciaparrone M, Cavanna L, Giuliani F, Maiello E, Testa A, Pederzoli P, Falconi M, Gallo C, Di Maio M, Perrone F, GOIM, GISCAD, GOIRC. Randomized phase III trial of gemcitabine plus cisplatin compared with single-agent gemcitabine as first-line treatment of patients with advanced pancreatic cancer: the GIP-1 study. J Clin Oncol 28, 1645-51 (2010). 
29. Philip PA, Benedetti J, Corless CL, Wong R, O’Reilly EM, Flynn PG, Rowland KM, Atkins JN, Mirtsching BC, Rivkin SE, Khorana AA, Goldman B, Fenoglio-Preiser CM, Abbruzzese JL, Blanke CD. Phase III study comparing gemcitabine plus cetuximab versus gemcitabine in patients with advanced pancreatic adenocarcinoma: Southwest Oncology Group-directed intergroup trial So205. J Clin Oncol 28, 3605-10 (2010).

30. Reni M, Cordio S, Milandri C, Passoni P, Bonetto E, Oliani C, Luppi G, Nicoletti R, Galli L, Bordonaro R, Passardi A, Zerbi A, Balzano G, Aldrighetti L, Staudacher C, Villa E, Di Carlo V. Gemcitabine versus cisplatin, epirubicin, fluorouracil, and gemcitabine in advanced pancreatic cancer: a randomised controlled multicentre phase III trial. Lancet Oncol 6, 369-76 (2005).

31. Conroy T, Desseigne F, Ychou M, Couche O, Guimbaud R, Becouarn Y, Adenis A, Raoul JL, Gougou-Bourgade S, de la Fouchardiere C, Bennouna J, Bachet JB, Khemissa-Akouz F, Pere-Verge D, Delbaldo C, Addenat E, Chauffert B, Michel P, Montoto-Grillot C, Ducreux M, Groupe tumeurs digestives of unicancer, PRODIGE intergroup. FOLFIRINOX versus gemcitabine for metastatic pancreatic cancer. $N$ Engl J Med 364, 1817-25 (2011).

32. Moore MJ, Goldstein D, Hamm J, Figer A, Hecht JR, Gallinger S, Au HJ, Murawa P, Walde D, Wolff RA, Campos D, Lim R, Ding K, Clark G, Voskoglou-Nomikos T, Ptasynski M, Parulekar W, National Cancer Institute of Canada Clinical Trials Group. J Clin Oncol 25, 1960-6 (2007).

33. Kadaba R, Birke H, Wang J, Hooper S, Andl CD, Di Maggio F, Soylu E, Ghallab M, Bor D, Froeling FEM, Bhattacharya S, Rustgi AK, Sahai E, Chelala C, Sasieni P, Kocher HM. Imbalance of desmoplastic stromal cell numbers drives aggressive cancer process. $J$ Pathol 230(1),107-17 (2013). 
34. Rucki AA, Zheng L. Pancreatic cancer stroma: understanding biology leads to new therapeutic strategies. World J Gastroenterol 20(9), 2237-46 (2014).

35. Neesse A, Michl P, Frese KK, Feig C, Cook N, Jacobetz MA, Lolkema MP, Buchholz M, Olive KP, Gress TM, Tuveson DA. Stromal biology and therapy in pancreatic cancer. Gut 6o(6), 861-8 (2011).

36. Bausch, D., et al. Plectin-1 as a novel biomarker for pancreatic cancer. Clin. Cancer Res. 17, 302-309 (2011).

37. Rosca, E.V., et al. Anti-angiogenic peptides for cancer therapeutics. Curr. Pharm. Biotechnol. 12, 1101-1116 (2011).

38. Wang, J. J., et al. Screening peptides binding specifically to colorectal cancer cells from a phage random peptide library. Asian Pac. J. Cancer Prev. 13, 377-381 (2012).

39. Reynolds F, Panneer N, Tutino CM, Wu M, Skrabal WR, Moskaluk C, Kelly KA. A functional proteomic method for biomarker discovery. PLoS One 6(7), e22471 (2011).

40. Ong SE, Biagoev B, Kratchmarova I, Kristensen DB, Steen H, Pandey A, Mann M. Stable isotope labeling by amino acids in cell culture, SILAC, as a simple and accurate approach to expression proteomics. Mol Cell Proteomics 1(5), 376-86 (2002).

41. Phillips P. Pancreatic stellate cells and fibrosis. In: Grippo PJ, Munshi HG editors. Pancreatic Cancer and Tumor Microenvironment. Trivandrum (India): Transworld Research Network; 2012. Chapter 3. Available from: http://www.ncbi.nlm.gov/books/NBK98937/ 
42. Dimou A, Syrigos KN, Saif MW. Overcoming the stromal barrier: technologies to optimize drug delivery in pancreatic cancer. Ther Adv Med Oncol 4(5), 271-9 (2012).

43. Chie WC, Chang YH, Chen HH. A novel method for evaluation of improved survival trend for common cancer: early detection or improvement of medical care. $J$ Eval Clin Prac 13(1), 79-85 (2007).

44. Howlader N, Noone AM, Krapcho M, Garshell J, Miller D, Altekruse SF, Kosary CL, Yu M, Ruhl J, Tatalovich Z,Mariotto A, Lewis DR, Chen HS, Feuer EJ, Cronin KA (eds). SEER Cancer Statistics Review, 1975-2011, National Cancer Institute. Bethesda, MD, http://seer.cancer.gov/csr/1975_2011/, based on November 2013 SEER data submission, posted to the SEER web site, April 2014.

45. Chari ST, Leibson CL, Rabe KG, Ransom J, de Andrade M, Petersen GM. Probability of pancreatic cancer following diabetes: a population based study. Gastroenterology 129(2), 504-511 (2005).

46. Brentnall TA. Management strategies for patients with hereditary pancreatic cancer. Current Treatment Options Oncology 6(5), 437-45 (2005).

47. Brand RE, Lynch HT. Hereditary pancreatic adenocarcinoma. A clinical perspective. The Medical Clinics of North America 84(3), 665-75 (2000).

48. Lichtenstein P, Holm NV, Verkasalo PK, Iliadou A, Kaprio J, Koskenvuo M, Pukkala E, Skytthe A, Hemminki K. Environmental and heritable factors in the causation of cancer--analyses of cohorts of twins from Sweden, Denmark, and Finland. The New England Journal of Medicine 343(2), 78-85 (2000).

49. Fong PY, Fesinmeyer MD, White E, Farin FM, Srinouanprachanh S, Afsharinejad Z, Mandelson MT, Brentnall TA, Barnett MJ, Goodman GE, Austin MA. 
Association of diabetes susceptibility gene calpain-10 with pancreatic cancer among smokers. Journal of Gastrointestinal Cancer 41(3), 203-8 (2010).

50. Peng $\mathrm{P}$, et al. Impact of sarcopenia on outcomes following resection of pancreatic adenocarcinoma. J Gasterointest Surg 16(8), 1478-86 (2012).

51. Slomka P. TH-CD-210-02: Advances in SPECT, SPECT/CT and PET/CT for cardiology. Med Phys 42(6), 3738 (2015).

52. Hammond ME, Lapointe GR, Feucht PH, Hilt S, Gallegos CA, Gordon CA, Giedlin MA, Mullenbach G, Tekamp-Olson P. IL-8 induces neutrophil chemotaxis predominantly via type I IL-8 receptors. J Immunol 155(3), 1428-33 (1995).

53. Rajagopalan L, Rajarathnam K. Ligand selectivity and affinity of chemokine receptor CXCR1. Role of N-terminal domain. J Biol Chem. 279(29), 300oo-8 (2004).

54. Van Damme J, Decock B, Conings R, Lenaerts JP, Opdenakker G, Billiau A. The chemotactic activity for granulocytes produced by virally infected fibroblasts is identical to monocyte-derived interleukin 8. Eur J Immunol 19(7), 1189-94 (1989).

55. Stadtmann A, Zarbock A. CXCR2: from bench to bedside. Front Immunol 3, 263 (2012).

56. Lazennec G, Richmond A. Chemokines and chemokine receptors: new insights into cancer-related inflammation. Trends Mol Med 16(3), 133-44 (2010).

57. Duell EJ, Casella DP, Burk RD, Kelsey KT, Holly EA. Inflammation, genetic polymorphisms in proinflammatory genes TNF-A, RANTES, and CCR5, and risk of pancreatic adenocarcinoma. Cancer Epidemiol Biomarkers Prev 15(4), 726-31 (2006). 
58. Hertzer KM, Donald GW, Hines OJ. CXCR2: a target for pancreatic cancer treatment? Expert Opin Ther Targets 17(6), 667-80 (2013).

59. Sparmann A, Bar-Sagi D. Ras-induced interleukin-8 expression plays a critical role in tumor growth and angiogenesis. Cancer Cell 6(5), 447-58 (2004).

6o. Wenger C, Ellenrieder V, Alber B, Lacher U, Menke A, Hameister H, Wilda M, Iwamura T, Beger HG, Adler G, Gress TM. Expression and differential regulation of connective tissue growth factor in pancreatic cancer cells. Oncogene 18(4), 107380 (1999).

61. Mazzocca A, Fransvea E, Dituri F, Lupo L, Antonaci S, Giannelli G. Downregulation of connective tissue growth factor by inhibition of transforming growth factor beta blocks the tumor-stroma cross-talk and tumor progression in hepatocellular carcinoma. Hepatology 51(2), 523-34 (2010).

62. Yang F, Tuxhorn JA, Ressler SJ, McAlhany SJ, Dang TD, Rowley DR. Stromal expression of connective tissue growth factor promotes angiogenesis and prostate cancer tumorigenesis. Cancer Res 65(19), 8887-95 (2005).

63. Waugh DJ, Wilson C. The interleukin-8 pathway in cancer. Clin Cancer Res 14(21), 6735-41 (2008).

64. Keefe, A. D., Pai, S., \& Ellington, A. Aptamers as therapeutics. Nat. Rev. Drug Discov. 9, 537-550 (2010).

65. Smith, G. P. \& Petrenko, V. A. Phage display. Chem. Rev. 97, 391-410 (1997).

66. Matochko, W. L., Cory Li, S., Tang, S. K., \& Derda, R. Prospective identification of parasitic sequences in phage display screens. Nucleic Acids Res. 42, 1784-1798 (2014). 
67. 't Hoen, P. A., et al. Phage display screening without repetitious selection rounds. Anal. Biochem. 421, 622-631 (2012).

68. Dias-Neto, E., et al. Next-generation phage display: integrating and comparing available molecular tools to enable cost-effective high-throughput analysis. PLoS One. 4, e8338 (2009).

69. Levitan B. Stochastic modeling and optimization of phage display. J Mol Biol $\mathbf{2 7 7}(4), 893-916$ (1998).

70. Derda R, Tang SK, Li SC, Ng S, Matochko W, Jafari MR. Diversity of phagedisplayed libraries of peptides during panning and amplification. Molecules 16(2), 1776-803 (2011).

71. Matochko WL, Chu K, Jin B, Lee SW, Whitesides GM, Derda R. Deep sequencing analysis of phage libraries using Illumina platform. Methods 58(1), 47-55 (2012).

72. Devlin, J. J., Panganiban, L. C., \& Devlin, P. E. Random peptide libraries: a source of specific protein binding molecules. Science 249, 404-406 (1990).

73. Kelly, K. A., et al. Detection of vascular adhesion molecule-1 expression using a novel multimodal nanoparticle. Circ. Res. 96, 327-336 (2005).

74. Nahrendorf, M., et al. Noninvasive vascular cell adhesion molecule-1 imaging identifies inflammatory activation of cells in atherosclerosis. Circulation 114, 15041511 (2006).

75. Nahrendorf, M., et al. 18F-4V for PET-CT imaging of VCAM-1 expression in atherosclerosis. JACC Cardiovasc. Imaging 2, 1213-1222 (2009).

76. Olumi AF, Grossfeld GD, Hayward SW, Carroll PR, Tisty TD, Cunha GR. Carcinoma-associated fibroblasts direct tumor progression of initiated human prostatic epithelium. Cancer Res 59(19), 5002-11 (1999). 
77. Qiu W, Hu M, Sridhar A, Opeskin K, Fox S, Shipitsin M, Trivett M, Thompson ER, Ramakrishna M, Gorringe KL, Polyak K, Haviv I, Campbell IG. No evidence of clonal somatic genetic alterations in cancer-associated fibroblasts from human breast and ovarian carcinomas. Nat Genet 40(5), 650-5 (2008).

78. Luga V, Wrana JL. Tumor-stroma interaction: revealing fibroblast-secreted exosomes as potent regulators of Wnt-planar cell polarity signaling in cancer metastasis. Cancer Res 73(23), 6843-7 (2013).

79. Augsten M. Cancer-associated fibroblasts as another polarized cell type of the tumor microenvironment. Front Oncol 4, 62 (2014).

8o. Bolte, S., \& Cordelieres, F. P. A guided tour into subcellular colocalizaton analysis in light microscopy. J. Microsc. 224, 213-232 (2006).

81. Marsh Rde W, Talamonti MS, Katz MH, Herman JM. Pancreatic cancer and FOLFIRINOX: a new era and new questions. Cancer Med 4, 853-863 (2015).

82. Brinton LT, Sloane HS, Kester M, Kelly KA. Formation and role of exosomes in cancer. Cell Mol Life Sci 72(4), 659-71 (2015).

83. Hakami AR, Ball JK, Tarr AW. Non-ionic detergents facilitate non-specific binding of M13 bacteriophage to polystyrene surfaces. $J$ Virol Methods 221: 1-8 (2015).

84. Kelly, K. A., Waterman, P., \& Weissleder, R. In vivo imaging of molecularly targeted phage. Neoplasia 8, 1011-1018 (2006).

85. Dasa SS, Suzuki R, Gutknecht M, Brinton LT, Tian Y, Michaelsson E, Lindfors L, Klibanov AL, French BA, Kelly KA. Development of target-specific liposomes for delivering small molecule drugs after reperfused myocardial infarction. $J$ Control Release Epub ahead of print (2015). 
86. Deshpande PP, Biswas S, Torchilin VP. Current trends in the use of liposomes for tumor targeting. Nanomedicine (Lond) 8(9), 1509-28 (2013).

87. Vasquez KO, Casavant C, Peterson JD. Quantitative whole body biodistribution of fluorescent-labeled agents by non-invasive tomographic imaging. PLoS One 6(6), e20594 (2011).

88. Gabizon A, Shmeeda H, Barenholz Y. Pharmacokinetics of pegylated liposomal Doxorubicin: review of animal and human studies. Clin Pharmacokinet 42(5), 41936 (2003).

89. McDonald DM, Baluk P. Significance of blood vessel leakiness in cancer. Cancer Res 62(18), 5381-5 (2002).

90. Beech JR, Shin SJ, Smith JA, Kelly KA. Mechanisms for targeted delivery of nanoparticles in cancer. Curr Pharm Des 19(37), 6560-74 (2013).

91. DeVita VT Jr, Chu E. A history of cancer chemotherapy. Cancer Res 68(21), 864353 (2008).

92. Mukherjee, S. (2010). The emperor of all maladies: A biography of cancer. New York: Scribner.

93. Barenholz Y. Doxil@--the first FDA-approved nano-dug: lessons learned. $J$ Control Release 160(2), 117-34 (2012).

94. FDA approves new treatment for advanced pancreatic cancer. FDA News Release. 22 October 2015. Available: http://www.fda.gov/NewsEvents/Newsroom/PressAnnouncements/ucm468654.h tm

95. Miller DR. A tribute to Sidney Farber-the father of modern chemotherapy. $\mathrm{Br} J$ Haematol 134(1), 20-6 (2006). 
96. Ryan DP, Hong TS, Bardeesy N. Pancreatic adenocarcinoma. N Engl J Med. 371, 1039-1049 (2014).

97. Matthaei H, Schulick RD, Hruban RH, Maitra A. Cystic precursors to invasive pancreatic cancer. Nat Rev Gastroenterol Hepatol 8(3), 141-50 (2011).

98. Gentile, J. R. 2004. Assessing fundamentals in every course through mastery learning." In Alternative strategies for evaluating student learning, Edited by: Achacoso, M. V. and Svinicki, M. D. 15-20. San Francisco: Jossey Bass.

99. Roediger III, H.L., \& Karpicke, J.D. The power of testing memory: basic research and implications for educational practice. Perspect. Psychol. Sci. 1, 181-210 (2006).

100. Roediger III, H.L. \& Butler, A.C. The critical role of retrieval practice in long-term retention. Trends Cogn. Sci. 15, 20-27 (2011).

101. Larsen, D.P., Butler, A.C., \& Roediger III, H.L. Test-enhanced learning in medical education. Med. Educ. 42, 959-966 (2008).

102. Custers, E.J.F.M. Long-term retention of basic science knowledge: a review study. Adv. In Health Sci. Educ. 15, 109-128 (2010).

103. SEER Cancer Statistics Factsheets: Pancreas Cancer. National Cancer Institute. Bethesda, MD, http://seer.cancer.gov/statfacts/html/pancreas.html

104. Wilentz RE, Iacobuzio-Donahue CA, Argani P, McCarthy DM, Parsons JL, Yeo CJ, Kern SE, Hruban RH. Loss of expression of Dpc4 in pancreatic intraepithelial neoplasia: evidence that DPC4 inactivation occurs late in neoplastic progression. Cancer Res. 6o(7), 2002-6 (2000).

105. Hudson, D. \& Scornavacca C. Dendroscope 3: An interactive tool for rooted phylogenetic trees and networks. Syst. Biol. 61, 1061-1067 (2012). 
106. Beech, J.R., Shin, S.J., Smith, J.A., \& Kelly, K.A. Mechanisms for targeted delivery of nanoparticles in cancer. Curr. Pharm. Des. 19, 6560-6574 (2013).

107. Goldman, L.W. Principles of CT and CT technology. J. Nucl. Med. Technol. 35, 115128 (2007).

108. Lammertsma, A.A. PET/SPECT: functional imaging beyond flow. Vision. Res. 41, 1277-1281 (2001).

109. Tonkens, R. An overview of the drug development process. Physician Exec. 31, 4852 (2005).

110. Glasier, A. \& Baird, D. The effects of self-administering emergency contraception. N. Engl. J. Med. 339, 1-4 (1998). 
APPENDIX 1

NGSanalyze code 


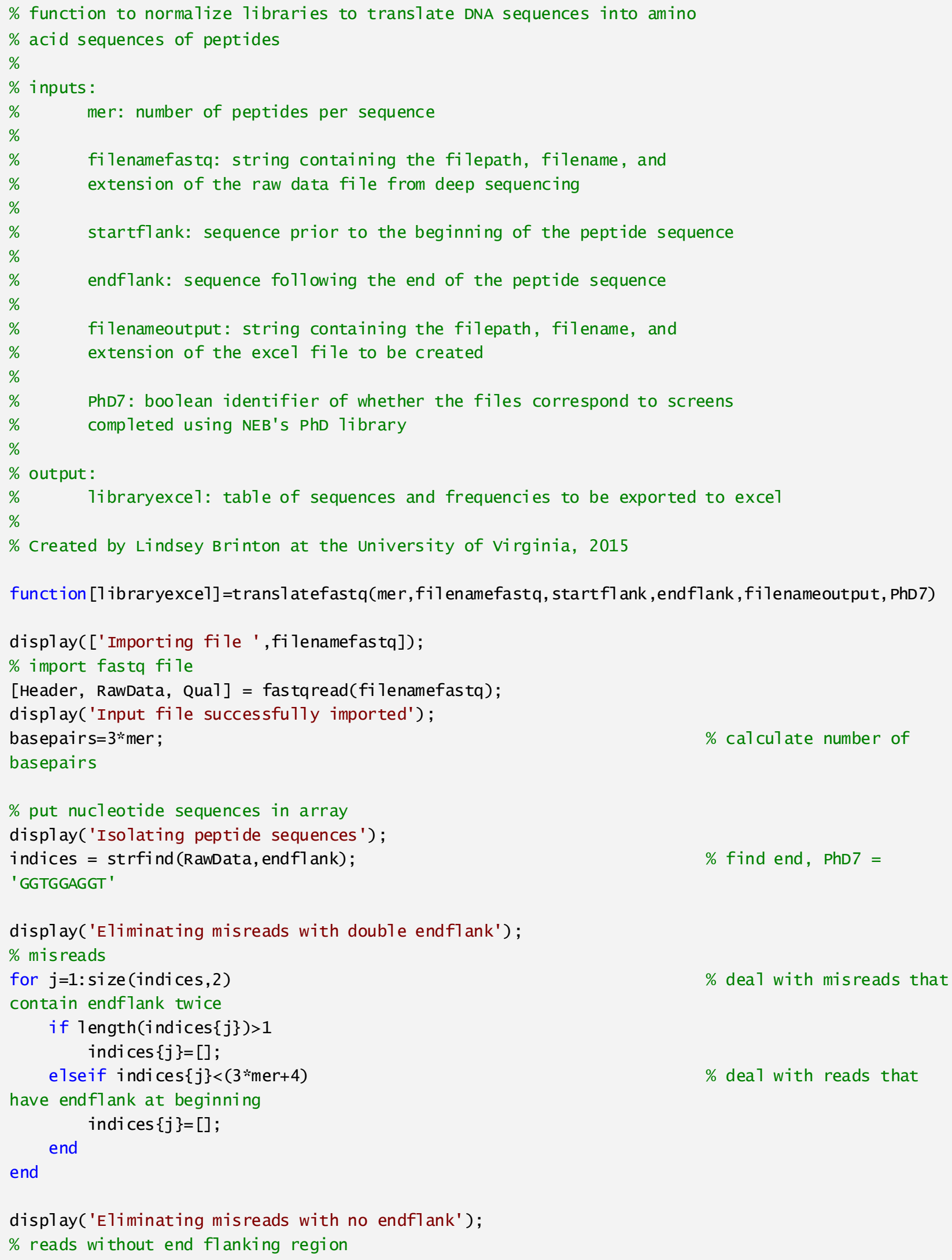




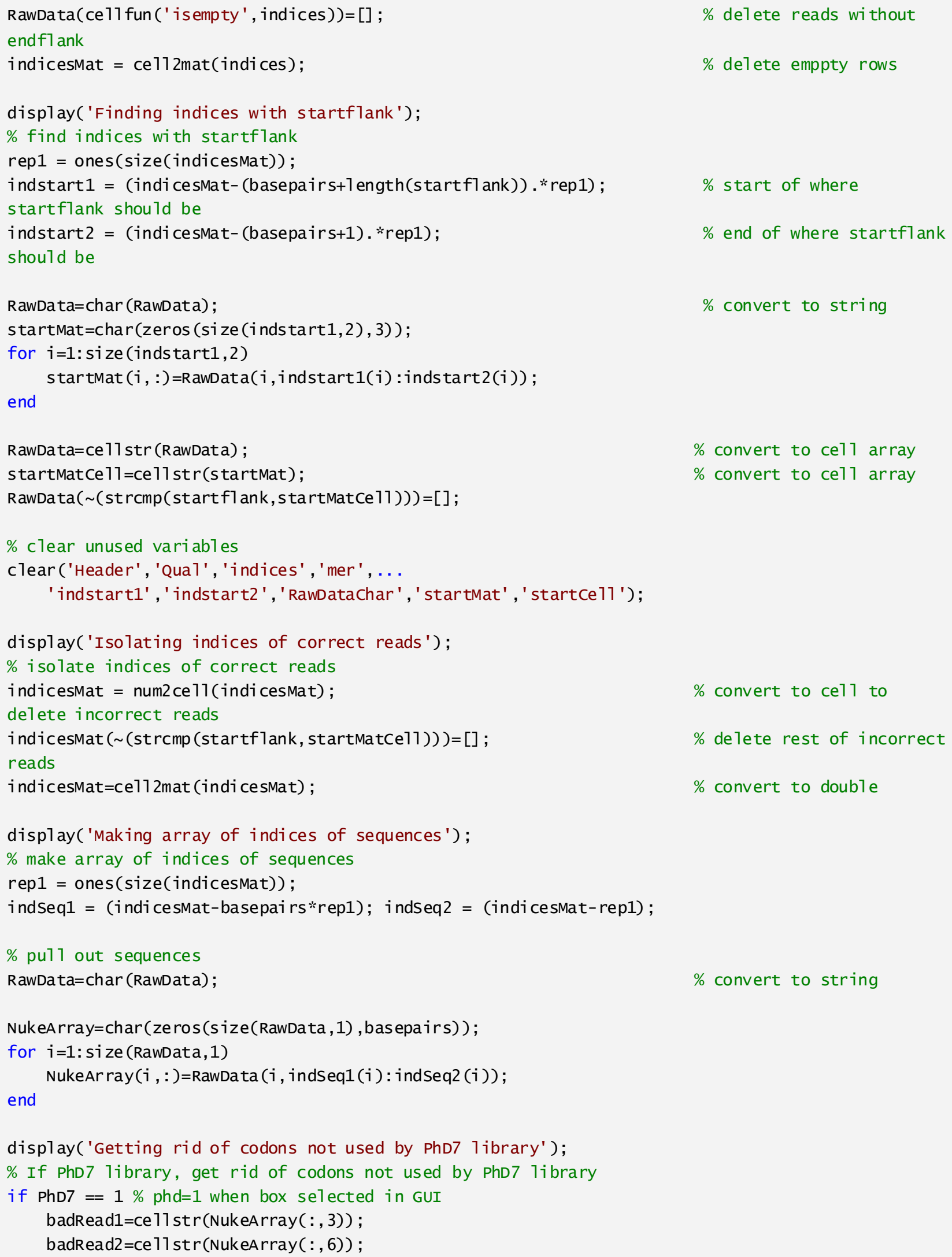

\% convert to cel1 array

$\%$ convert to cel1 array

$\%$ delete rest of incorrect

$\%$ convert to double 
badRead3=ce11str(NukeArray $(:, 9))$;

badRead4=ce11 str(NukeArray $(:, 12))$;

badRead5=ce $11 \operatorname{str}($ NukeArray $(:, 15))$;

badRead6=ce11 str (NukeArray $(:, 18))$;

badRead7=ce11 str(NukeArray $(:, 21))$;

badReadMatrix $=[\operatorname{strcmp}($ 'A' , badRead1), $\operatorname{strcmp}($ 'A', badRead2) , . .

$\operatorname{strcmp}($ 'A' , badRead3), strcmp ( 'A' , badRead4), strcmp ('A' , badRead5), ...

strcmp ('A', badRead6), strcmp( 'A' , badRead7) , ...

strcmp ( 'C', badRead1), strcmp ( 'C', badRead2) , . .

strcmp ('C', badRead3), strcmp ( 'C', badRead4), strcmp ('C', badRead5), ...

strcmp ( 'C' , badRead6), strcmp ( 'C' , badRead7)];

[brRow brCo1]=find (badReadMatrix);

$\%$ find indices of

instances of bad reads

NukeAr ray=cel1str(NukeArray);

NukeAr ray (uni que(brRow)) $=[]$;

$\%$ convert to ce11 array

$\%$ delete bad codon reads

e1se

NukeAr ray=cel 1str(NukeArray);

\% convert to ce11 array end

\% clear unused variables

clear ('RawData' , ' indseq1' , 'indi cesmat' , 'basepai rs ', ' rep1' , ' indseq2 ' , . . .

'RawDatachar2 ', 'indices2', 'brco1', ' brRow', 'badRead1', ' badRead2' , ...

' badRead3' , 'badRead4 ' , 'badRead5' , 'badRead6' , 'badRead7' , 'badReadMatrix') ;

$\%$ convert to amino acids

display ('Converting to amino acid sequences') ;

AAarray = nt2aa(NukeArray, 'A7 ternativestartCodons ', fal se, 'ACGTonly', false);

AAarray = regexprep (AAar ray, ' *' , 'Q') ;

$\%$ replace stop (*) with

(Q)

clear ('NukeArray');

display ('Determining frequencies');

$\%$ determine frequencies

tableAA = tabulate(AAarray);

tableAA = sortrows (tableAA, 2);

\% calculate frequencies

SeqFreqTable = tableAA $(:, 1: 2)$;

$\%$ sort table

$\%$ sequences

\% display stats

display (['Number of total valid reads: ',num2str(size(AAarray,1))]);

display (['Number of unique reads: ', num2str(size(SeqFreqTable,1))]);

clear ('tableAA' , 'AAarray');

display('starting export') ;

$\%$ export to excel

iterationsXLS $=\operatorname{cei1}(\operatorname{size}(($ SeqFreqTable $), 1) /(1000000)) ; \quad$ Determine if for 1oops

necessary

$\mathrm{p}=1$;

\% initialize counter

if iterationsXLS $==1$

display('Exporting to exce1: one sheet');

[status1, message1]=x1swri te (filenameoutput, SeqFreqTable $(1: 1$ ength (SeqFreqTable),: ),1); \% write excel file--> only le6 rows each sheet

else 


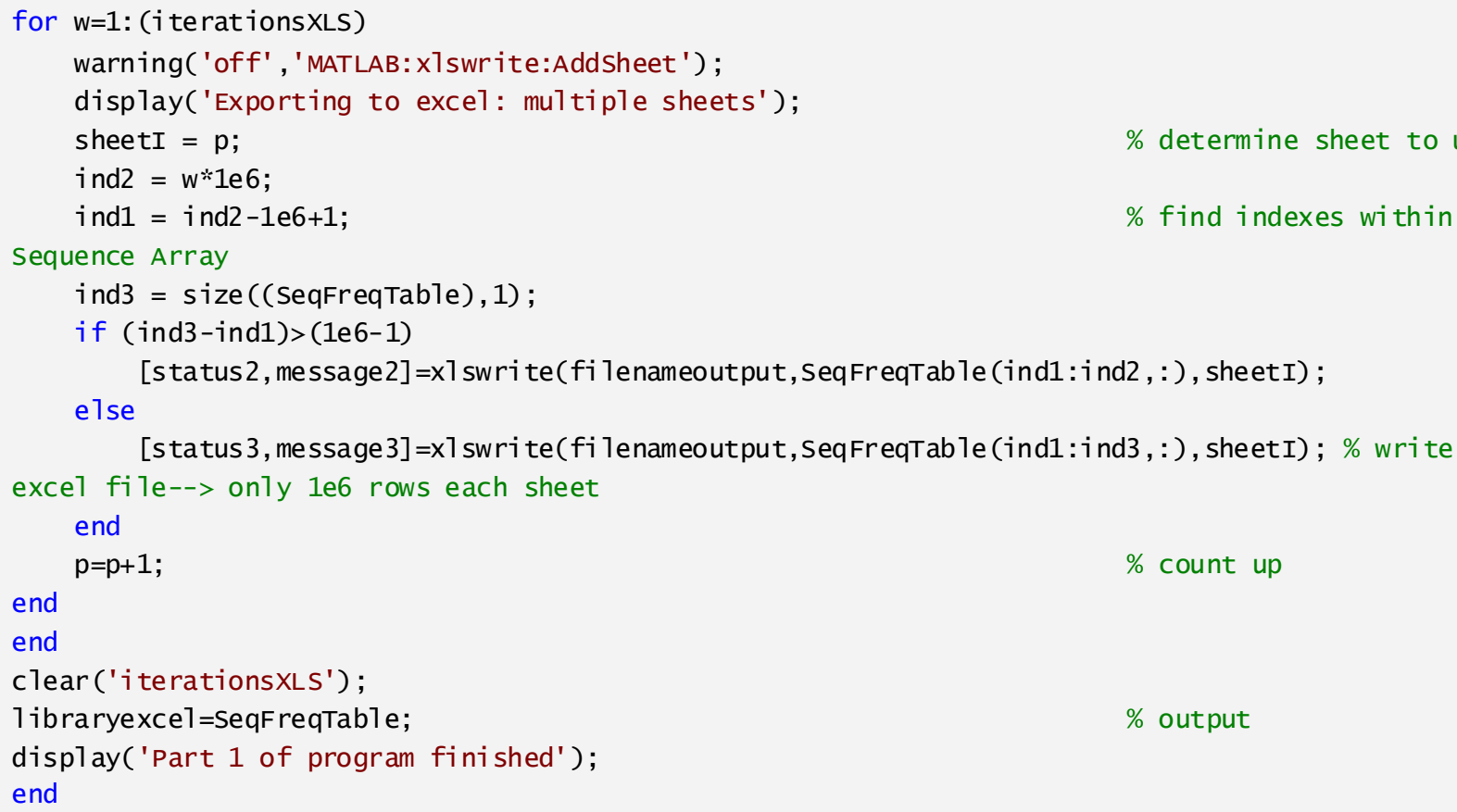

Published with MATLAB ${ }^{\circledR}$ R2014a 


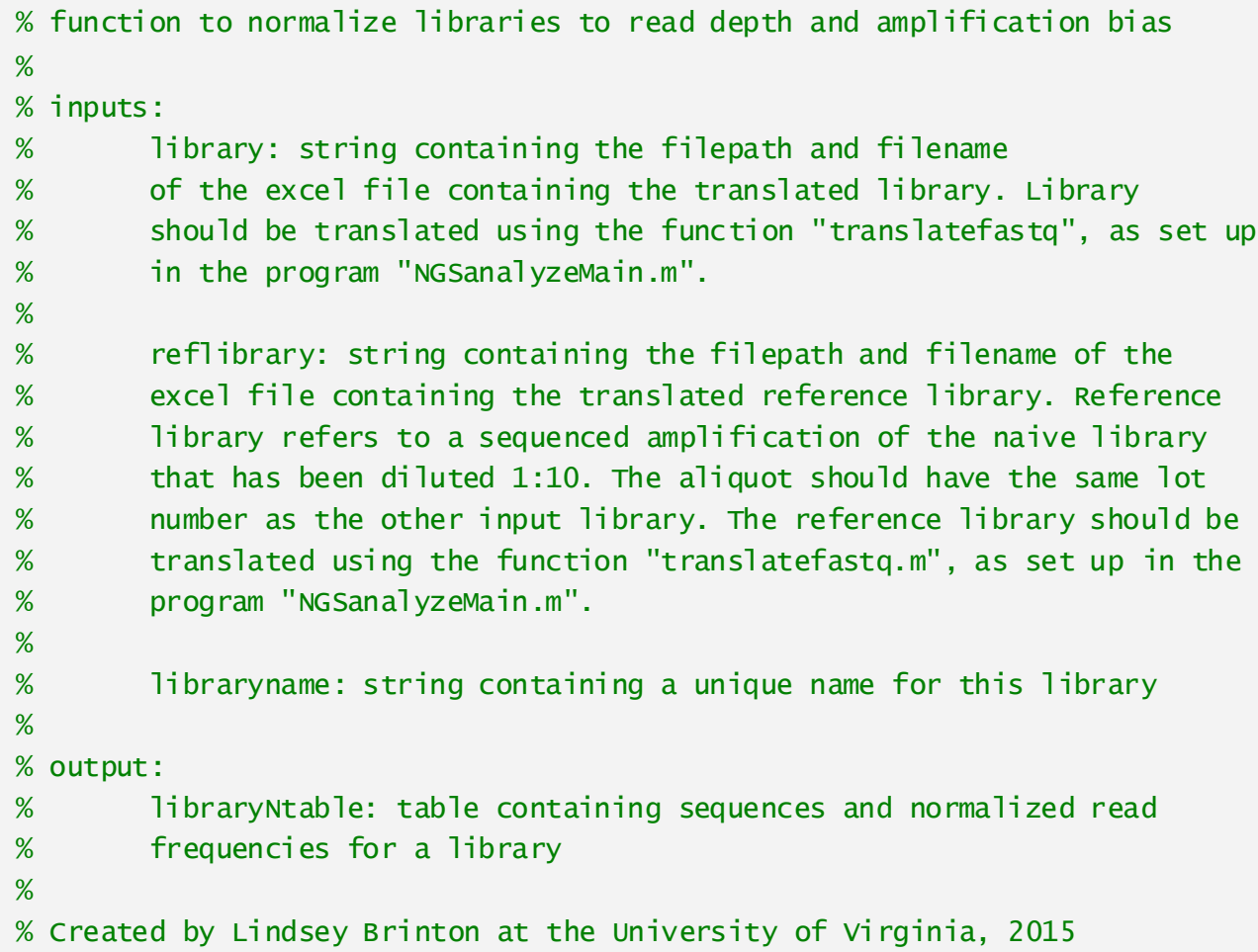

function [1 ibraryNtable]=norma 7 ize 1 ibrary (1ibrary, reflibrary, 1 ibraryname)

display ('starting 7ibrary normalization');

\% create table for reference library

$[x, 1 i b r e f 1 a, r]=x 1$ sread $\left(\right.$ reflibrary, 'A: $\left.A^{\prime}\right)$;

[1ibref1b] = x1 sread(reflibrary, 'B:B') ;

tableref1 = table(1ibrefla,1ibref1b, 'Variablenames', \{'Peptide ', 'RefLibrary' \});

$\%$ create table for library 1

$[x, 1 i b l a, r]=x 1$ sread $\left(1 i b r a r y,{ }^{\prime} A: A^{\prime}\right)$;

$[1 \mathrm{ib} 1 \mathrm{~b}]=\mathrm{x} 1 \mathrm{sread}\left(1 \mathrm{ibrary}, \mathrm{B}^{\mathrm{B}} \mathrm{B}\right.$ ');

table1 = table(1ib1a,1ib1b, 'Variablenames', \{'Peptide ', 'Library' \});

\% join the reference 1ibrary and 1ibrary together

[mergelibrary,a,b]=outerjoin(tableref1, table1, 'Mergekeys ', true);

\% clear extra variables

clear ('x', '1ibref1a ', 'r', '1ibref1b', 'tab1eref1', 'x', '1ib1a' , 'r' , '1ib1b');

clear('table1', 'a', 'b');

$\%$ convert table to two arrays

Seqs $=$ table2array (mergelibrary $(:,\{$ 'Peptide' $\})) ; \%$ array of strings (sequences)

Nums $=$ table2array (mergelibrary $(:, 2:$ end)); $\quad \%$ array of doubles (read frequencies)

\% separate reference library and library

readlengths $=[\operatorname{nansum}(\operatorname{Nums}(:, 1))$, nansum $(\operatorname{Nums}(:, 2))] ; \quad \%$ get readlengths before changes

$\%$ only keep sequences with frequencies $>10$ in 1ibrary 


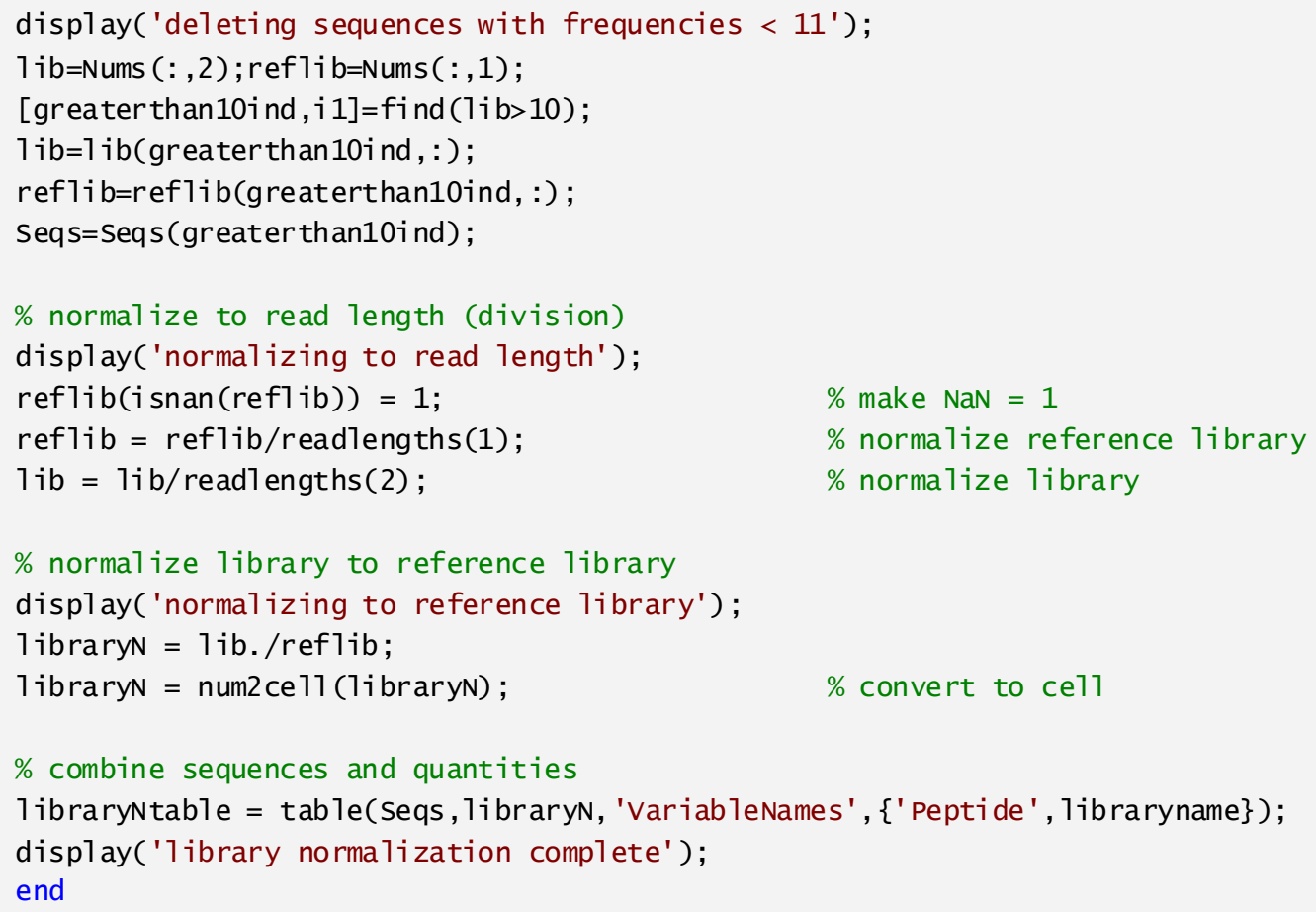

Published with MATLAB ${ }^{\circledR}$ R2014a 


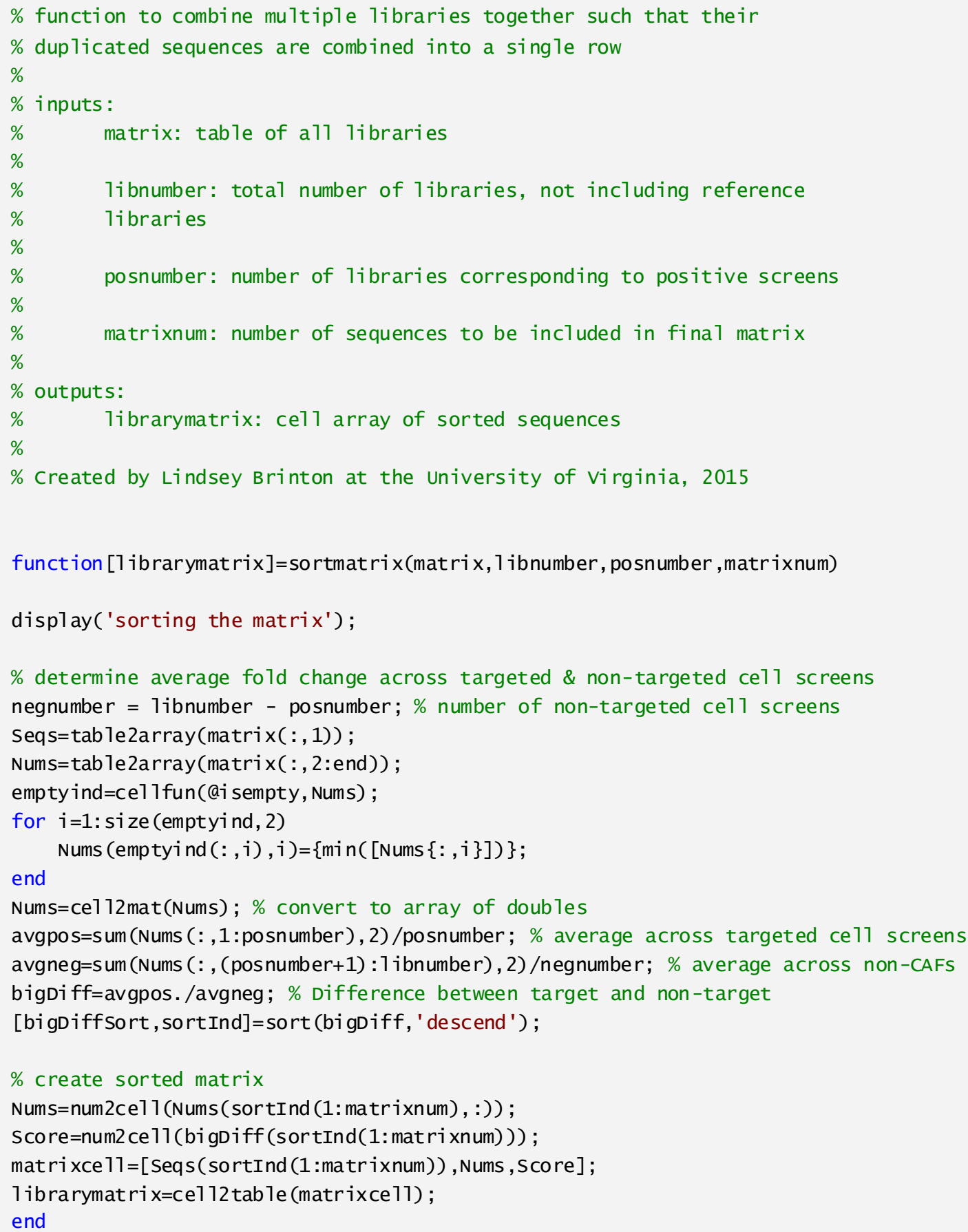

Published with MATLA $B^{\circledR}$ R2014a 


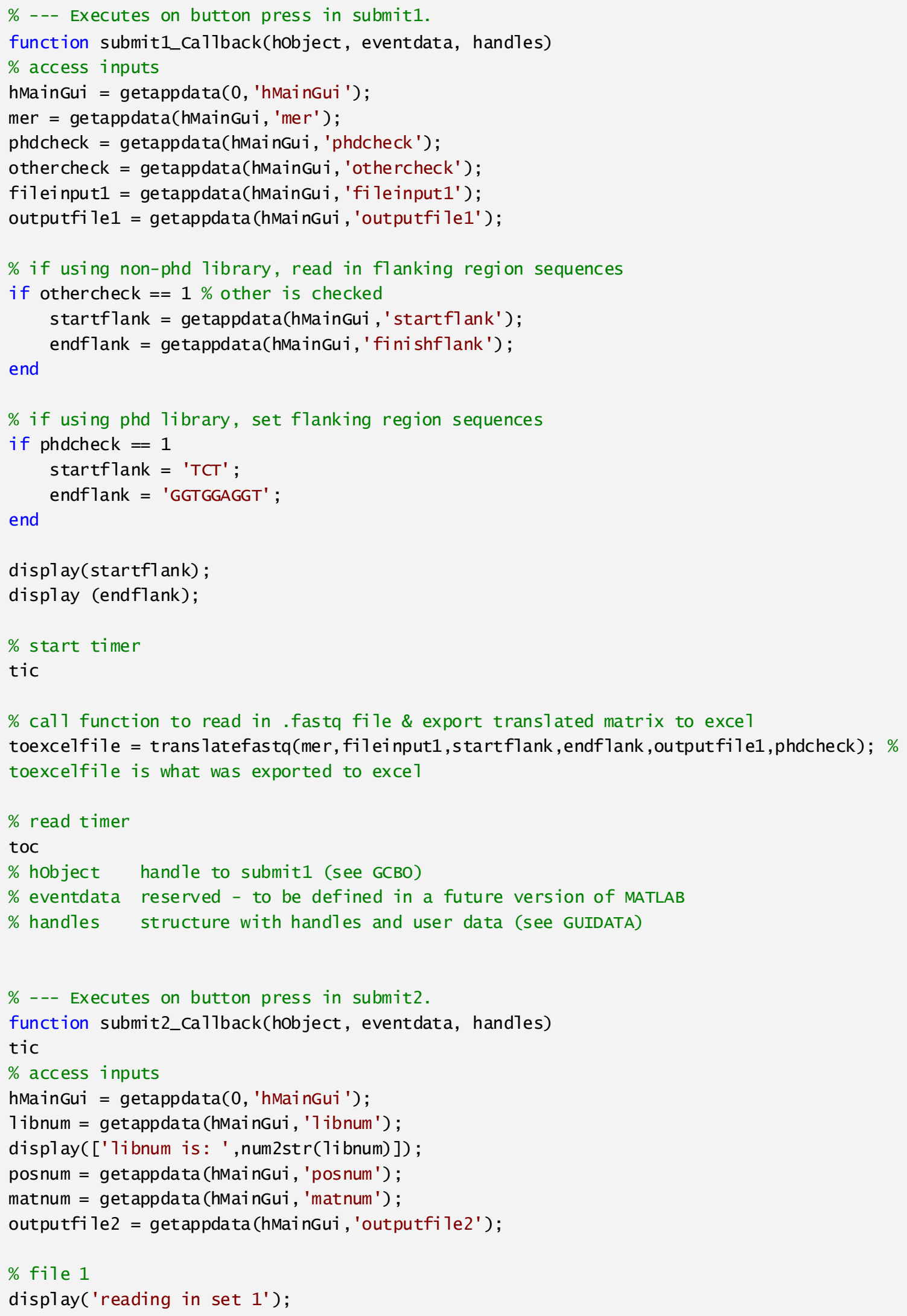







1ibfile6 = getappdata(hMainGui, ' '1ibfile6');

reffile6 = getappdata(hMainGui, ' reffile6');

1ibrary6table = normalizelibrary (1ibfile6, reffile6, ' 1ibsix');

[E, ie,je]=outerjoin(D, 1ibrary6table, 'Mergekeys', true); \% add file 6

clear('ie', 'je', 'D', '7ibrary6table');

al1together $=\mathrm{E}$;

end

$\%$ file 7

if 7 ibnum $>6$

display ('adding set 7 ');

1ibfile7 = getappdata(hMaingui, ' 1ibfile7');

reffile7 = getappdata(hMainGui, ' reffile7');

1 ibrary7table = normalizelibrary (1ibfile7, reffile7, ' 1ibseven');

$[F, i f f, j f]=$ outerjoin (E, 1ibrary7table, 'Mergekeys', true); \% add file 7

clear('iff' , 'jf', 'E' , ' 1ibrary7tab7e');

a)1 together $=F$;

end

$\%$ file 8

if 7 ibnum $>7$

display ('adding set 8 ');

1ibfile8 = getappdata(hMaingui, ' 1ibfile8');

reffile 8 = getappdata(hmainGui, ' reffile 8 ');

1ibrary8table = normalizelibrary (1ibfile8, reffile8, ' '1ibEight');

[G,ig,jg]=outerjoin(F, 1ibrary8tab1e, 'Mergekeys', true); \% add file 8

clear('ig', 'jg' , 'F', '7ibrary8table');

a) 1 together $=\mathrm{G}$;

end

$\%$ file 9

if 7 ibnum $>8$

display ('adding set 9');

1ibfile9 = getappdata(hMainGui,' '1ibfile9');

reffile9 = getappdata(hMainGui, 'reffile9');

1 ibrary9table = normalizelibrary (1 ibfile9, reffile9, '1ibnine');

[H,ih,jh]=outerjoin(G, library9table, 'Mergekeys', true); \% add file 9

clear('ih', 'jh', 'G', '7ibrary9table');

al1together $=\mathrm{H}$;

end

$\%$ file 10

if 1 ibnum $>9$

display('adding set $10^{\prime}$ );

1 ibfi 1e10 = getappdata (hMainGui, ' 1 ibfi 1e10');

reffile10 = getappdata (hMainGui, ' reffile10');

1 ibrary10table = norma1ize1 ibrary (1ibfi 1e10, reffile10, '1ibTen');

$[\mathrm{I}, \mathrm{i} i, \mathrm{ji}]=$ outerjoin( $\mathrm{H}, 1 \mathrm{ibrary} 10 \mathrm{tab}$ le, 'MergeKeys',true); \% add fi le 10 clear('ij', 'ji', 'H', '7ibrary10table')

al1together=I ;

end 


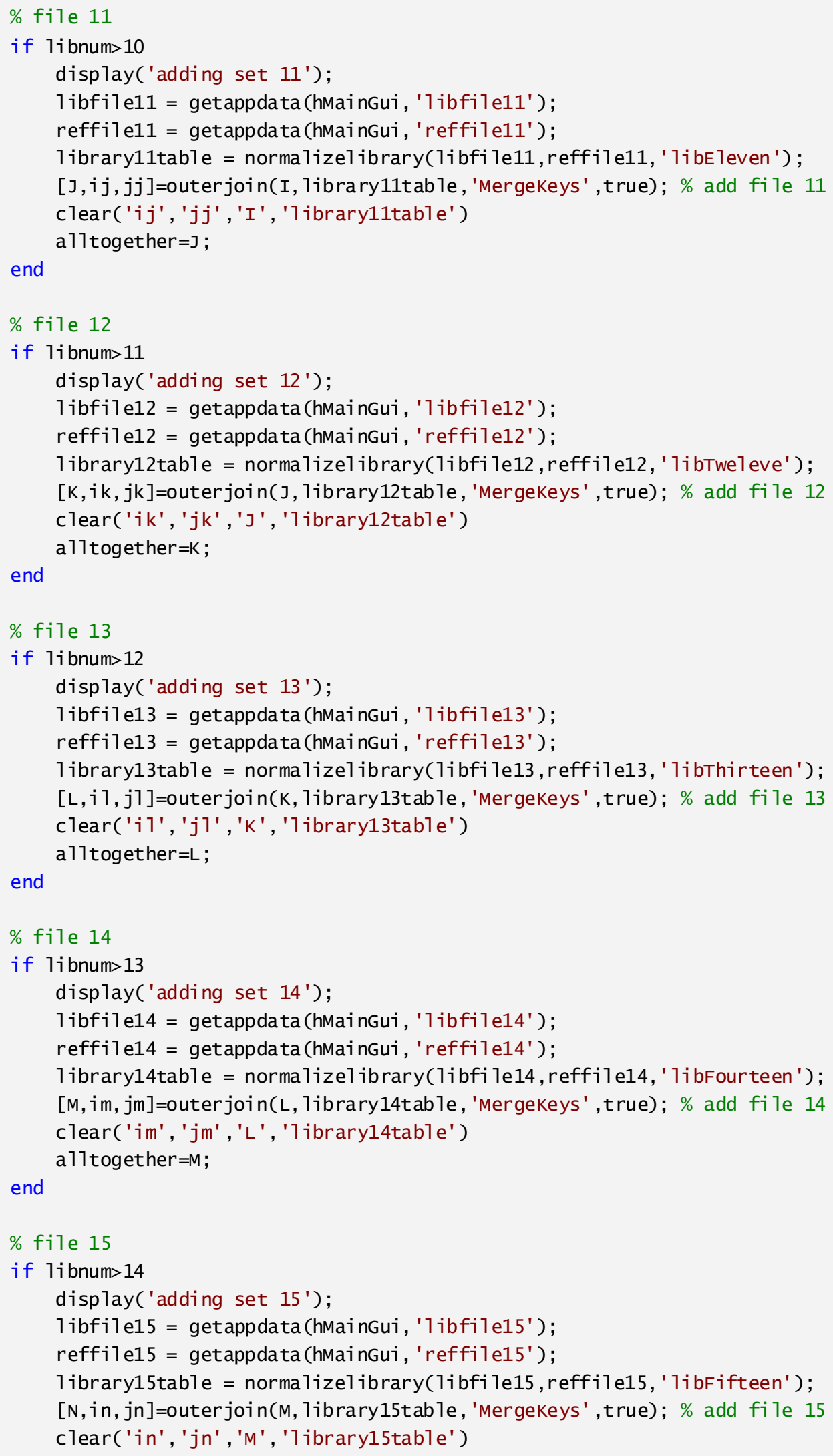




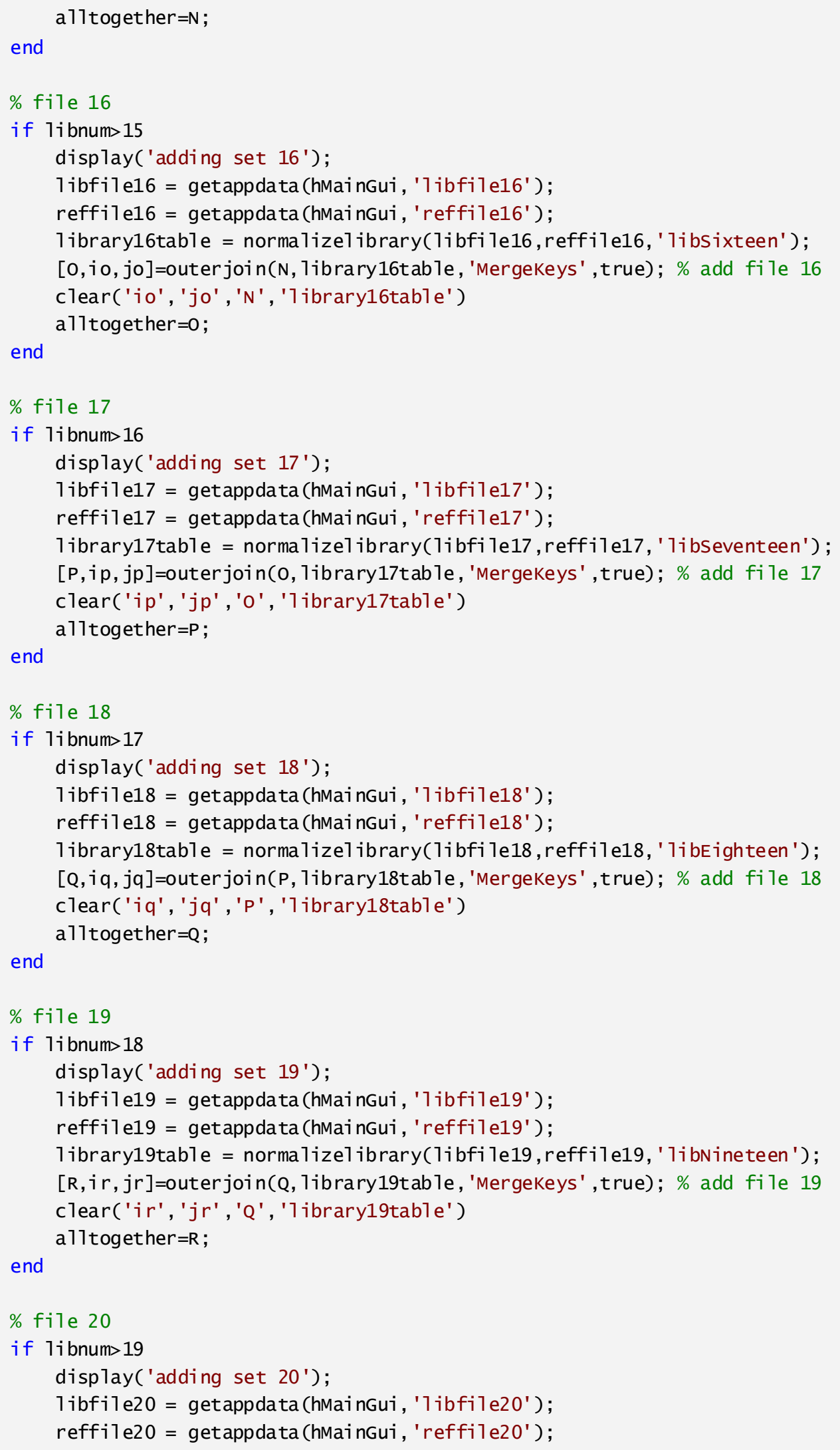




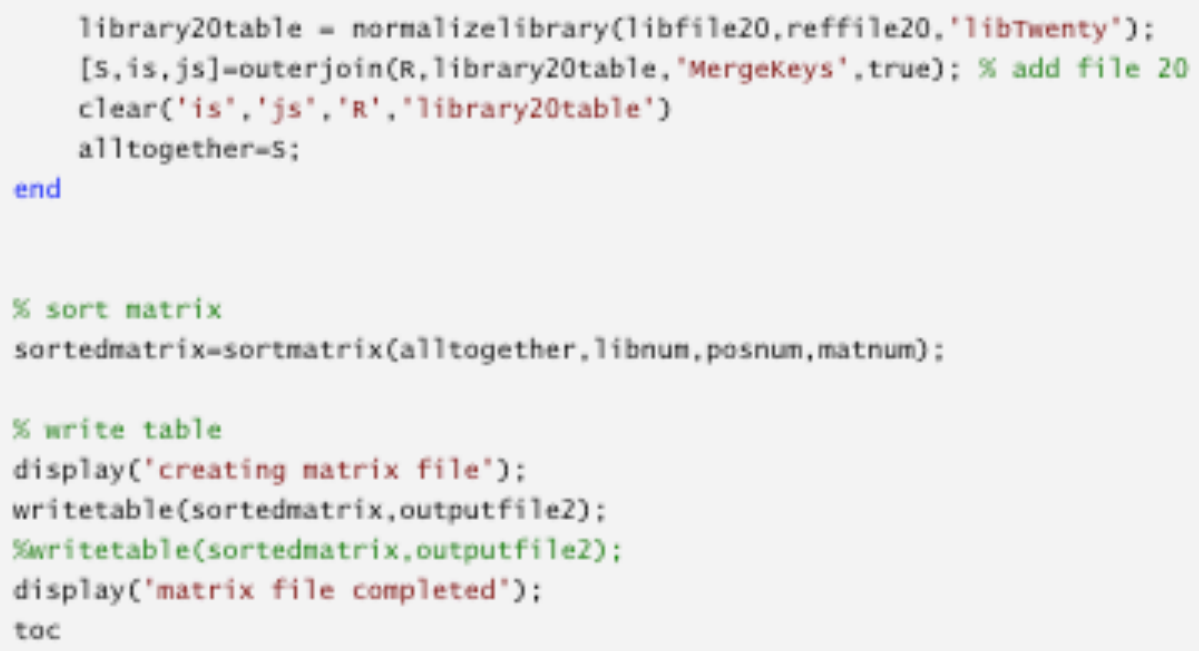


APPENDIX 2

Innovations in

teaching

nanomedicine

Reprinted from Brinton, L. T., \& Curley, C. T., \& Kelly, K. (2015, June), The LAWA Technique Implemented in a Course in Nanomedicine Paper presented at 2015 ASEE Annual Conference and Exposition, Seattle, Washington. 10.18260/p.24892 with (C) 2015 American Society for Engineering Education cited as per policy. 
The LAWA technique implemented in a course in nanomedicine

Advanced engineering topics are essential to a complete undergraduate engineering education. In biomedical engineering, advanced topics often require integration of many fields of study, understanding of the technical aspects of wet-lab experimentation methods, and relating mathematical concepts to biological systems - making such topics particularly challenging to teach. To help meet this challenge, we have developed and evaluated the lecture - assessment - worksheet - article (LAWA) technique in an upperlevel elective course titled "Nanomedicine" in the Department of Biomedical Engineering at the University of Virginia (UVA). We hypothesized that this method would increase understanding and improve student performance as measured by comparison of graded assessments from the redesigned course with those of previous years when the course was taught in a traditional lecture format. Topics in nanomedicine included the design of nanoparticle drug delivery systems, molecular imaging, and materials biocompatibility. To understand these topics, students had to learn more than simply the facts about existing nanoparticles and nanotechnologies; they also needed to learn how to apply relevant knowledge from previous courses in the basic sciences (biology, chemistry, physics, biotransport) and basic mathematics (algebra, calculus, differential equations) to address disease-specific problems. Students were additionally required to make engineering design choices compatible with current laboratory experimental techniques and consider constraints like cost, manufacturability, and FDA regulations as other influential factors. Students were challenged to expand their knowledge base through in class note taking but importantly, they also had to critically read relevant journal articles (some provided by the instructor and others self-identified).

In this paper, we first describe the course organization and the LAWA technique, which presents context-specific knowledge in the form of a lecture, encourages students to 
study that material and recall it in a quiz, has students work on group activities to apply and build on that knowledge, then has them read a related research article. We also discuss participation-based assessment that focuses on completion rather than accuracy, which we implemented for all of the worksheets. In addition to comparing performance on graded assessments of the redesigned and traditionally formatted course, we gain insight into the efficacy of these teaching tools via multiple forms of anonymous student feedback and a mid-semester Teaching Analysis Poll, where the instructors exited the classroom and trained staff from UVA's Teaching Resource Center elicited feedback from students about their learning in the course in a confidential and interactive way. We found that LAWA increased understanding and development of independent learning skills, and generated more interest and excitement about nanomedicine among the students.

\section{Course Organization}

While many university classes aim to teach a large amount of information each semester, we decided to scale-down the amount of information we covered in order to allow for more time to cover each topic. Overlearning has been shown to be less dominant (yet helpful) in long-term retention, but essential to achieving a certain level of mastery [98]. Therefore, we systematically reiterated the most important concepts by relating them back to the specific content we were teaching each week. Additionally, we took advantage of the testing effect to have students retrieve information multiple times and in different formats (frequent quizzes, tests, worksheets, presentation question and answer sessions, debates) to promote long-term retention [99 100]. The midterm and final exams tested material that had already been assessed by quizzes, but were intended to enhance learning by spacing retrieval over time (a.k.a. distributed practice) and included nonmultiple choice questions to require effortful recall, which has more benefit to the 
students' retention [101]. We strongly encouraged participation since, as the name suggests, active learning elements (worksheets, presentations, discussions, debates) are most effective when students actively take part in them. As instructors, we consistently encouraged our students both verbally and through emails to participate in class and allotted 10 percent of the final course grade to participation. We encouraged students who are introverted to find another way to show us that they were interacting with the material and making an effort. We designed one project to provide some breadth, but the main focus of the course was to have students truly understand the most important concepts. In other words, rather than being able to remember certain formulas in the future, we wanted students to be able to look up formulas and still understand what they mean. The course was organized based on three learning objectives. Students should be able to:

1. identify and apply the important considerations for drug design, including the ability to design mathematical models of drug transport

2. recognize areas where nanomedicine could be used

3. talk intelligently about nanotechnologies

Our style became LAWA, where at times the worksheet was replaced by a project or debate. Table 1 shows the concepts that we covered with their related quiz topics, active learning elements, and associated journal article.

Objective one: Identify and apply the important considerations for drug design, including the ability to design mathematical models of drug transport. We used lectures and worksheets to accomplish this objective. To guide students in the important elements of drug design, we devised lectures around each concept breaking the concepts up into smaller parts. Students were quizzed on the main concepts from each lecture the 
next class period, which encouraged them to spend time learning and understanding the material. Students would then complete an in-class worksheet that required them to build off of their base, explore new concepts, and apply the drug design elements previously covered. Worksheets were graded on completion and effort so that students could be creative and focus on the process of drug design instead of the "right answers." Alternatively, we sometimes had students do a project or participate in an in-class debate in lieu of a worksheet to accomplish a similar goal of deepening their understanding of the material and getting a chance to apply their knowledge.

Objective two: recognize areas where nanomedicine could be used. Journal articles were assigned as homework to accomplish this objective. The articles reinforced the concepts from class by explaining them in a different way. Each article was selected because it directly showed application of the concept in nanomedicine research (Table A2.1). The articles had the added benefit of also requiring the students to analyze data and concepts critically.

Objective three: talk intelligently about nanotechnologies. For this objective, we assigned two projects and held two in-class debates. The first project divided students into teams and assigned them a type of nanoparticle or nanotechnology. This survey familiarized students with many disparate types of nanomedicine research and also made them very knowledgeable about one type. These presentations generated more excitement about nanomedicine because students were able to see the cool and creative cutting-edge research. The journal articles assigned prepared them for this project. For the second project, we divided students into teams, assigned them each a disease, and told them to design a nanoparticle or nanotechnology to ameliorate that disease. We lectured on presentation skills as they applied to talking about nanomedicine research, then paired two groups and had them take turns practicing presenting and offering 
feedback. Students were informed that how they presented would be a portion of their grade. Additionally, we held two in-class debates that encouraged students to learn about some of the social and ethical issues that influence nanomedicine research.

\begin{tabular}{|c|c|c|c|}
\hline Lecture Concepts & Quiz Topics & Worksheet/Project/Debate & Journal Article \\
\hline $\begin{array}{l}\text { pharmacokinetics, } \\
\text { pharmacodynamics, } \\
\text { compartment models }\end{array}$ & $\begin{array}{l}\text { meaning of } \\
\text { equations, } \\
\text { assumptions, } \\
\text { biological } \\
\text { relevance of } \\
\text { variables }\end{array}$ & $\begin{array}{l}\text { worksheet: designing a } \\
\text { compartment model for a } \\
\text { specific disease }\end{array}$ & $\begin{array}{l}\text { Pharmacokinetics of } \\
\text { pegylated liposomal } \\
\text { Doxorubicin. [88] }\end{array}$ \\
\hline $\begin{array}{l}\text { In vivo nanoparticle } \\
\text { characteristics, } \\
\text { targeted drug } \\
\text { delivery, PBPK } \\
\text { models }\end{array}$ & $\begin{array}{l}\text { biodistribution, } \\
\text { digestion \& } \\
\text { absorbance of } \\
\text { drugs, interaction } \\
\text { of nanoparticles } \\
\text { and immune } \\
\text { system, toxicity, } \\
\text { clearance } \\
\text { pathways from } \\
\text { body }\end{array}$ & $\begin{array}{l}\text { worksheet: going from } \\
\text { simple compartment } \\
\text { models to PBPK models } \\
\text { worksheet: designing a } \\
\text { liposome that incorporates } \\
\text { Eribulin }\end{array}$ & $\begin{array}{l}\text { Mechanisms for } \\
\text { targeted delivery of } \\
\text { nanoparticles in } \\
\text { cancer [106]. }\end{array}$ \\
\hline $\begin{array}{l}\text { types of nanoparticles } \\
\& \text { nanotechnologies }\end{array}$ & $\begin{array}{l}\text { basic descriptions, } \\
\text { special properties }\end{array}$ & $\begin{array}{l}\text { presentation: general } \\
\text { information, how it works, } \\
\text { applications, advantages } \\
\text { and disadvantages }\end{array}$ & $\begin{array}{l}\text { Selected by } \\
\text { students to prepare } \\
\text { for presentations }\end{array}$ \\
\hline $\mathrm{CT}$ and $\mathrm{MRI}$ imaging & $\begin{array}{l}\text { imaging planes, } \\
\text { spin, types of } \\
\text { relaxation images, } \\
\text { contrast agents, } \\
\text { image } \\
\text { reconstruction, } \\
\text { collimation }\end{array}$ & $\begin{array}{l}\text { worksheet: starting with the } \\
\text { first generation of CT and } \\
\text { re-evolving to the reach } \\
\text { current CT technology } \\
\text { debate: should low-dose } \\
\text { computed tomography lung } \\
\text { cancer screening be } \\
\text { implemented as part of the } \\
\text { Medicare program }\end{array}$ & $\begin{array}{l}\text { Principles of CT and } \\
\text { CT technology [107] }\end{array}$ \\
\hline $\begin{array}{l}\text { PET and SPECT } \\
\text { imaging }\end{array}$ & $\begin{array}{l}\text { tracer decay and } \\
\text { detection, } \\
\text { functional vs. } \\
\text { anatomical } \\
\text { imaging, half-life, } \\
\text { sensitivity }\end{array}$ & $\begin{array}{l}\text { worksheet: creating a } \\
\text { textbook-style application } \\
\text { box that describes how } \\
\text { PET/SPECT use } \\
\text { radioactivity }\end{array}$ & $\begin{array}{l}\text { PET/SPECT: } \\
\text { functional imaging } \\
\text { beyond flow [108]. }\end{array}$ \\
\hline clinical trails & $\begin{array}{l}\text { phases, } \\
\text { randomization, } \\
\text { FDA approval, } \\
\text { ethical } \\
\text { considerations }\end{array}$ & $\begin{array}{l}\text { worksheet: designing a } \\
\text { clinical trial based on a } \\
\text { drug with promising } \\
\text { preclinical data }\end{array}$ & $\begin{array}{l}\text { An overview of the } \\
\text { drug development } \\
\text { process [109]. }\end{array}$ \\
\hline $\begin{array}{l}\text { drug study design } \\
\text { social and ethical } \\
\text { issues }\end{array}$ & $\begin{array}{l}\text { sample size, } \\
\text { measured } \\
\text { outcomes, } \\
\text { potential sources } \\
\text { of bias, study } \\
\text { length }\end{array}$ & $\begin{array}{l}\text { debate: based on a given } \\
\text { study, should emergency } \\
\text { contraception be available } \\
\text { over the counter }\end{array}$ & $\begin{array}{l}\text { The effects of self- } \\
\text { administering } \\
\text { emergency } \\
\text { contraception [110]. }\end{array}$ \\
\hline
\end{tabular}

Table A2.1: The LAWA approach. First, basic understanding of each group of concepts is achieved by a quiz. Next, the understanding of these concepts is deepened through completion of an in-class worksheet. Finally, a related journal article shows how those concepts have realworld application. 
1. $C=\mathrm{C}_{0} \mathrm{e}^{-\mathrm{ket}}$ should not be used to calculate concentration when (answer all that apply)

A. the system has heterogeneity

B. the drug takes effect immediately

C. the system is open

D. the drug accumulates over time in the area of interest

E. the system is homogenous

2. Which 2 of the following might occur if you underestimate the half-life of a drug?
A. effective dose won't be reached
B. toxic dose
C. overestimate the area under the curve
D. underestimate the area under the curve

Box A2.1: Sample concept-oriented multiplechoice questions. These questions test students' understanding of when a certain simplified equation can be applied and how the half-life of a drug is related to other pharmacokinetic variables.

\section{An example of the LAWA}

\section{format}

Here we illustrate an example

of our approach: we taught students in a lecture format about a standard onecompartment model for pharmacokinetic analysis. Their quiz focused on the concepts important to the connectivity of the models, important assumptions, and the equations they would be using (Box A2.1). The in-class worksheet then had them

apply this knowledge. First, they predicted input and output function characteristics and did simple calculations using the equations based on a one-compartment model. Next, they read a short paragraph about what compartments biologically represent, chose a disease and drew what they thought a three-compartment model might look like. This really assesses how well students grasp the connection between the model components and the biology. As the instructors walked around the room, we would ask probing questions about their choices. For example, "It looks like you chose to make compartments that each represent an organ of the body, could you explain what each arrow you drew represents biologically?" They were also prompted in the next section to make a few changes to their design based on the fact that most of the time a three- 
compartment model allows you to predict what is happening at the tissue and cell level from measurements taken from the blood; here, students realized that they must start with a plasma compartment and work their way physically from capillary to interstitial space to intercellular space. Finally, students wrote out equations for their threecompartment models using an example two-compartment model and the associated equations. Students would have to reverse engineer the way to develop equations using the two-compartment model example and then apply it to their own model, helping them to associate variables with model components. To demonstrate to students where compartment models are used in nanomedicine, they read the journal article Pharmacokinetics of Pegylated Liposomal Doxorubicin [88]. In figure six of this paper, a diagram of a three-compartment model shows how doxorubicin goes from the blood into the interstitial space while still in a liposome. Once it is in the interstitial space, the doxorubicin is released from the liposome and continues on into the tumor cells. Students could easily see the relevance of the class material to actual research.

\section{Comparison of the redesigned course with the former traditional style course}

In previous years, this class had been taught by one of the same co-instructors, covering many similar concepts. This traditional style included lecturing each class period, three exams, and a final project. Due to limited availability of previous examinations, we selected three true-or-false questions and two short-answer questions to repeat on quizzes in our redesigned course. Because these repeated questions differed in style from our quizzes, the questions were given as extra credit on a quiz. We defined a specific rubric for the short answer questions that was used to re-grade short answer questions from the traditionally taught course and the redesigned course. The rubric for the first short answer question contained five metrics and that of the second contained two 
metrics. Students from the traditional course performed slightly better on one of three true-false questions $(\mathrm{p}=0.03)$, while the other two true-false questions were not significantly different between the two groups ( $p>0.05$ ). The short answers were divided; students from the traditional course did better on the first short answer question ( $\mathrm{p}<0.03$ ), while students from the redesigned course scored higher on the second short answer question ( $\mathrm{p}<0.02$, Figure A2.1). Interestingly, in accordance with the student

a

\begin{tabular}{lll}
\hline & Traditional & Redesigned \\
\hline Year & 2009 & 2014 \\
Instructors & 1 & 2 \\
TAs & 1 & 1 \\
Students & 34 & 70 \\
\hline
\end{tabular}

C

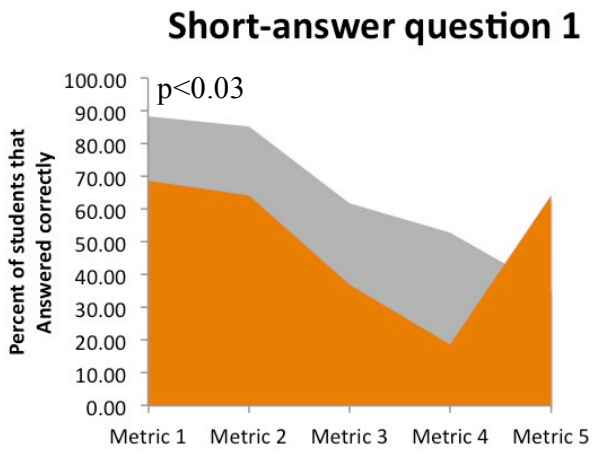

b

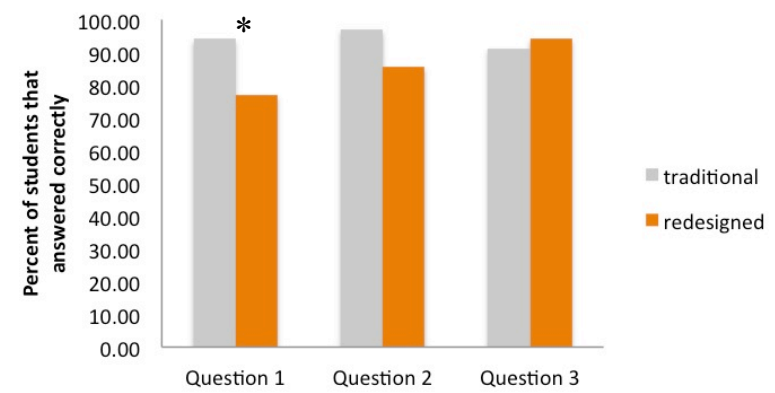

Short-answer question 2

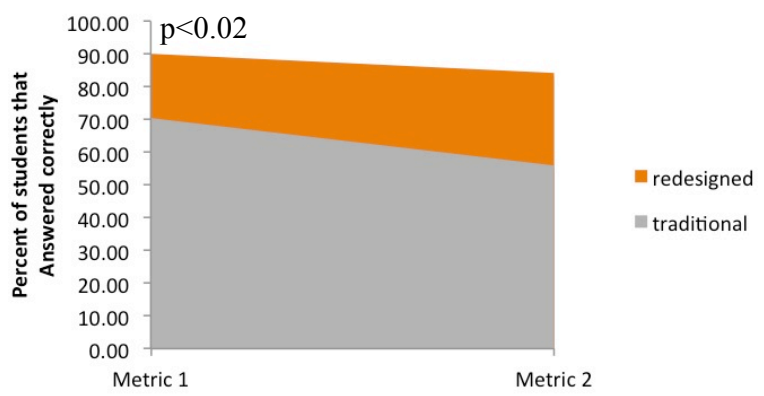

Figure A2.1: Quantitative comparison of student performance in traditional vs redesigned course. Students from the redesigned course were tested on three true-false questions and two short answer questions that had been used when the class was traditionally taught. The short answer questions from both types of course were evaluated with the same rubric (five metrics for the first short answer and two metrics for the second one). (a) This chart shows how the makeup of the classes differed. (b) Students from each course scored similarly on true-false questions, with the traditional type class outperforming the redesigned class on one out of three questions ( ${ }^{*}$ denotes statistical significance according to the chi-squared test, $p=0.03$ ). (c) Students taught traditionally scored higher on the first shortanswer question, while students from the redesigned course scored higher on the second short-answer question. The shape of the plotted points shows metric-to-metric variance in performance, and the area indicates overall performance on that question. All differences for the short-answer question metrics were statistically significant according to the chi-squared test. For short-answer question one, $p<0.03$ and for short-answer question two, $p<0.02$. 
feedback from the redesigned course about seeing the "big picture", we noticed that given the same short answer question prompt, "big picture" ideas were more likely to be described by students taught in the redesigned course; whereas, smaller details were more likely to be presented by students taught in the traditional course.

\section{Participation-based assessment from student feedback}

Engineers must solve problems by know the facts and equations and being able to apply them to the appropriate situation. Since facts and equations can be easily looked up, but understanding is a longer process that requires pondering, practicing, and often correction, we tried participation-based assessment. We hypothesized that grading the worksheets for completion would create an environment where students could toil with complex ideas and think of creative approaches to solving problems without being worried that it would negatively impact their grades. Thus, worksheets and debates were graded solely on completion and effort rather than accuracy.

We provided several channels for feedback from the students so we could make the necessary adjustments to make this tactic successful in helping students to learn. Students could send in anonymous feedback at any point during the course. We used this feature to request that students answer the following questions about midway through the semester and again at the end of the course:

1. In your opinion, what teaching tools are most and least beneficial to your learning (for example, the lectures, lecture recaps, frequent quizzes, the worksheets, small group discussions, large class discussions, in-class debates)? Please comment on how one or more teaching tools helps or impedes your learning. 
2. Do the worksheets increase or decrease your confidence in approaching nanomedicine-related questions?

3. Does it help you that the worksheets are graded for completion rather than accuracy?

Additionally, the Teaching Resource Center (TRC) polled the students in absence of the instructors about which parts of the class they felt were helping their learning and which parts were hindering it. We consistently made changes based on what the students answered and this feedback helped us to gain perspective on what the students' response. 33 percent of students responded to the mid-semester feedback request, 63 percent of students responded to feedback questions on the end-of-course evaluation, and all of the students present on the day of the TRC poll participated in that. Figure 2 summarizes the feedback from the students. The most frequently answered beneficial teaching tools were frequent quizzes (68\% average), lectures (55\% average), quiz preparation documents ( $44 \%$ average), and worksheets (36\% average). Students felt the least beneficial teaching tools were small group discussions (23\% average) and large class discussions (23\%). The students overwhelmingly responded that the worksheets increased their confidence in approaching nanomedicine-related questions (85\% average), with the remaining students responding that it did not alter their confidence one way or the other. Similarly, the vast majority of students (92\% average) felt that having their worksheets graded for completion rather than accuracy increased their learning.

Student comments helped us to determine if the participation-based worksheets did indeed create a more inventive environment where students could critically analyze new and complex problems. Students needed some time to get accustomed to participation- 
a
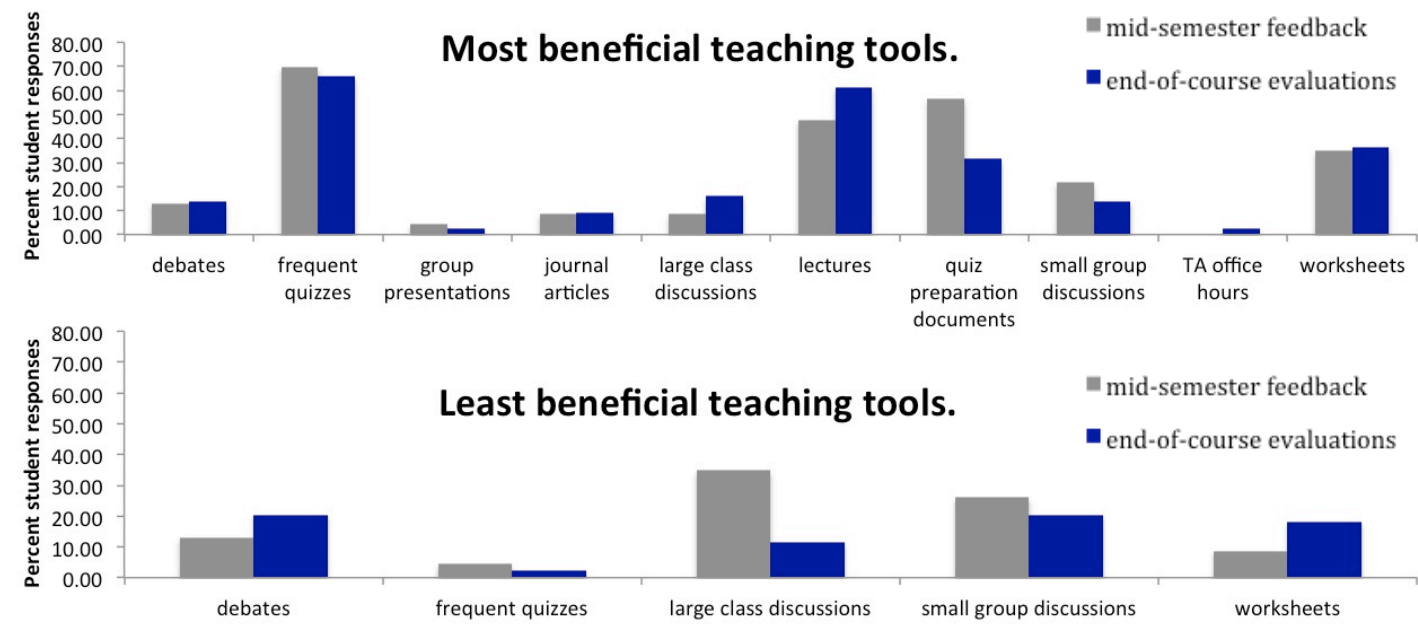

documents

b

Effect of worksheets on confidence in approaching nanomedicine questions.

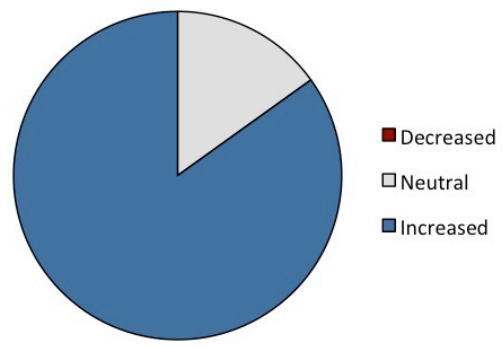

C Benefit of grading worksheets for completion rather than accuracy.

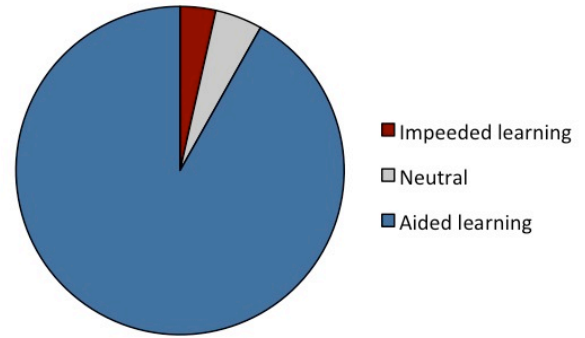

Figure A2.2: Student feedback. (a) Students were moderately consistent between their mid-semester and end-of-course feedback. Students felt that their learned profited most from the frequent quizzes, lectures, quiz preparation documents, and worksheets. They felt that their learning was most hindered by large and small discussions, with some students $(<20 \%)$ also feeling that the debates and worksheets were not helpful. (b) $85 \%$ of students said the worksheets increased their confidence in approaching nanomedicinerelated questions. (c) $92 \%$ of students credited participation-based grading with aiding their learning.

based grading. A student summarized, "Initially, we all felt so lost because we were used to having an exact correct answer, and we didn't know what the right answers were. Later we became used to the completion rather than accuracy system and I think it aided our learning a lot. It encouraged thinking and free class discussion without grade-related concern." Many of the students noted that it was less stressful to be graded by completion. The students found that they could focus on understanding the material instead of on getting a good grade. One student explained, "With the emphasis away 
from correct answers, I started to just explore the knowledge I obtained from the class and think about how I would apply it." Students also reported feeling more comfortable taking risks, seeing the "bigger picture", and not being afraid of being wrong on the first try. However, there were also some common criticisms. Multiple students said that the worksheets felt like "busy work" at times. A few students also recognized that the same system that allowed them the freedom to contemplate the material and focus on understanding it also made it possible to put in a sub-par level of effort. One student stated, "some students might try harder if they were graded for accuracy and would get more from them." Students also pointed out that the frequent quizzes fulfilled the role of grading for accuracy while the worksheets were completed when the information was still very new and that it would have felt unfair to be expected to get all of the answers correct. Several students agreed that increasing instructor-led discussion of the worksheets would make them more effective and wished that there had been a smaller instructor-to-student ratio (we had two instructors, one teaching assistant (TA), and 70 students).

\section{Participation in class weakly correlated with student performance on graded assessments}

The TA kept track of participation points for each class and provided students with summaries of their level of participation during the semester as a checkpoint. We hypothesized that a higher level of participation would lead to better grades and a better understanding of the material. To test our hypothesis, we compared the average participation level as determined by participation points to average exam scores, average quiz scores, and final project grades. We found that high participation had a weak positive correlation with average exam scores $(\mathrm{p}=0.06)$ and final project grades $(\mathrm{p}=0.05$, 
$\mathrm{a}$

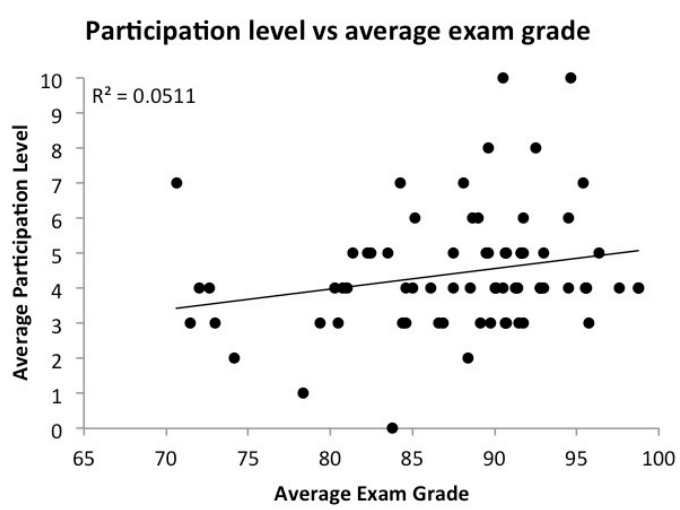

b

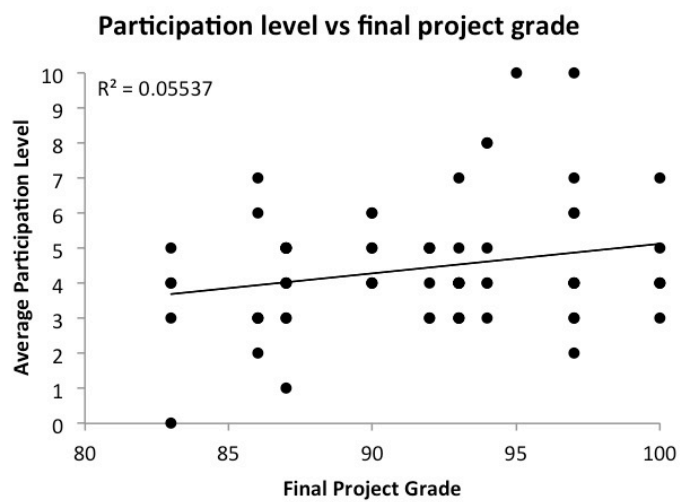

Figure A2.3: Correlation between level of participation and grades. It appears that participating more in class did help students to receive higher grades on their exams and final projects. There was no correlation between level of participation and average quiz grades. (a) There was a weak positive correlation $(p=0.06)$ between how much a student participated in class and his or her average exam grade. (b) There was a weak positive correlation $(p=0.05)$ between how much a student participated in class and his or her final project grade. P-values were determined using a linear regression t-test.
Figure A2.3). Therefore we concluded that participation can lead to better grades in classes that employ active learning techniques.

\section{Conclusions and lessons}

\section{learned}

Worksheets and participationbased assessment can be effective. Worksheets can be a useful teaching tool, but are difficult to create. We found that some of our worksheets were well received and others did not accomplish our goals. The greatest difficulty was in writing questions that were challenging and would push the students, but also not so hard that students felt incapable and frustrated. We

identified a few common themes among the less successful worksheets: (1) they assumed more background knowledge than students remembered, (2) the questions were so open-ended that students were unsure where to begin, and (3) the questions were too obvious so students felt like they were childish. We recommend carefully gauging students' level of knowledge and exposure to the material in writing worksheets. In the 
case of the more successful worksheets, students appreciated the learning opportunity provided by participation-based assessment. We were especially able to see great depth of knowledge during the final projects. In addition to presenting sophisticated ideas, students were able to intellectually defend the ideas and their design decisions during the question and answer portion.

Review relevant material from other courses. Nanomedicine builds on the foundation laid by several other courses. Our listed prerequisites include cell and molecular biology and biomechanics. Additionally, we expect students to be familiar with ordinary differential equations, mathematical modeling, and basic physics. About half of the students have also taken a course in biotransport. Certainly it is not possible to reteach the building blocks from these courses, but we found that students had forgotten much of the material and needed more review than we had anticipated. For example, students had difficulty grasping drug clearance concepts because they no longer remembered the difference between monoexponential and biexponential decay. It has been suggested that advanced courses should begin by reviewing the information from other courses that students will need and that instructors should design the course to "retroactively reinforce the consolidation of knowledge accumulated in previous courses" [102]. As part of the poll conducted mid-semester by the TRC, students explained that the instructors assumed they had more background knowledge than they did for certain graphs and equations. We recommend figuring out the essential pieces of background information and incorporating a review of them into the course.

Student evaluations did not reflect quality of presentations. In an effort to help students pay closer attention to their peers' presentations and provide peer feedback to students, we had each student fill out a short evaluation after each presentation. Students circled on a scale of one to five the slide quality, verbal content, and presentation skills, and 
listing one thing that was well done and one thing that needed improvement. We then compiled the results of the evaluations for each group. In a side-by-side comparison of the peer evaluations and the instructor evaluations, there was little correspondence. In fact, there was very little variation at all between the student evaluations, indicating that the students may not have wanted to be overly harsh with each other or perhaps did not put forth the effort to give a good evaluation. In comparing and contrasting the various comments, we found that many students said the same criticism over and over for each group. Additionally, the comments were very contradictory, making it less constructive as peer feedback for the students. Although different circumstances or different types of peer evaluations may enhance learning, we concluded that these student evaluations were not helpful and discontinued their use.

Resistance to new learning techniques. This course was taught to upper-level students who have specific expectations for course format, namely lectures and tests. Therefore, they were very uncomfortable with the format at the beginning of the semester. This was clearly reflected in the student feedback that we received. A typical student comment was "at first, worksheets were strenuous but it helped in applying learned material from class." We found that explaining the reasoning behind doing a certain activity made the students more open and willing to give it a serious effort. We recommend having a high level of transparency when trying out new teaching methods in upper-level courses.

Class discussions. We used two types of discussions: (1) large class discussion in which all of the students could raise their hands and ask questions or comment, and (2) small group discussions during lectures where students were asked to think about a questions and then discuss it with a partner or in a group of three or four students (usually $3-5$ minutes). Both of these discussions were unpopular with the students. The most common reason given was that it was too difficult to figure out the most important and 
correct information from large group discussions and that the small group discussions often got tangential. As part of the poll conducted mid-semester by the TRC, students explained that the discussions were too long and that they wanted really clear answers that they could use to study for quizzes and tests. As a result of the mid-semester feedback, we discontinued the large group discussions. We believe that this is why fewer students listed large group discussions as least beneficial teaching tools at the end of the semester compared to midway through (Figure A2.2A). In contrast, we tried to improve the small group discussions by shortening them and giving more hints. This resulted in only slightly less students (20\% compared to $26 \%$ ) naming small group discussions as a less helpful teaching method at the end of the semester compared to midway through (Figure A2.2A). End-of-the-course evaluations revealed that students had a hard time staying on track, and we recommend displaying a slide with the questions that are to be discussed as well as some thoughts to get students started if small group discussions are used.

The lecture - quiz - activity - journal article (LAWA) method. Overall, this style of teaching seemed to help students to gain good understanding of the most important concepts. Students performed well on these concepts on quizzes and tests, and were able to apply them on worksheets and in projects. This style of teaching did not seem to improve student answers on specific questions compared to a previous traditionally taught semester. However, students clearly went beyond rote memorization in interacting with the material and demonstrated impressive critical thinking of drug design in their final projects. We also found an unexpected outcome: increased interest and excitement about nanomedicine. We had students staying after class to discuss different things they learned in class. Students would email us about news articles they saw that involved nanomedicine or tell us about times in other classes that they used 
knowledge from this class. Five of our students started volunteering in labs to do nanomedicine research. Though not formally a part of our course objectives, this signified to us that these teaching tools were successful. 


\section{Document A2.1 Example worksheet}

We have talked extensively about the reasons for using nanoparticles for drug delivery, especially in the context of cancer. There are two dominating reasons for using this type of delivery system. The first we saw when we studied doxorubicin/Doxil. Describe critical toxicity in your own words and how it relates to doxorubicin/Doxil.

Eribulin is currently in a clinical trial for liposomal incorporation for treatment of metastatic breast cancer, yet the toxicity profile of Eribulin has been shown to be manageable. Postulate why it would be beneficial to incorporate this drug into a liposome.

For the purposes of this worksheet, you are the lead designer of a liposome that will incorporate Eribulin. Based on class discussions of size, charge, and hydrophobicity, what characteristics would you want your liposome to have? Justify your selections.

Did you remember to consider RES in your design? If not, what changes would you make?

Draw a schematic of your liposome. Be sure to include: (1) the basic bilayer of the liposome, (2) aqueous soluble drug trapped in the liposome interior, (3) lipid soluble drug trapped in the lipid membrane, (4) PEGylation, (5) attachment of a targeting ligand.

What advantages are there to having both (2) and (3)?

What is the purpose of (4)? 
Imagine a targeting strategy for your liposome, remembering that you are trying to reach metastatic breast cancer cells. What disease characteristics would influence this strategy? How are you going to target these cells?

Congratulations! Your liposome synthesis was successful and now you want to validate whether or not your liposomes are working. Write out hypotheses that you would need to test in order to be confident that your liposomes are going to do exactly what you want them to!

I hate to be the bearer of bad news, but your experiments show that the spleen is getting a toxic dose of Eribulin. What can you do about this? First think about changes you might make in the liposomes themselves. Now think about changes in dosing and include a graph of concentration versus time for your toxic liposome dosing and altered non-toxic liposome dosing.

Your liposome is looking really great thanks to the alterations that you made, but you want to know that the pharmacokinetics look like so you decide to fit a model to data that you get in a study with rats. Draw a diagram of the PBPK model that you would like to use to represent your liposomes in rats. For one organ, illustrate what the 3-comparment model would look like and write out equations. 


\section{Document A2.2: Example project guidelines}

Create a presentation in which you detail the design of a nanoparticle for your assigned disease (lung cancer, liver cancer, diabetes, bone infection, or myocardial infarction). You will have 10 minutes for your presentation plus 5 minutes for questions. You must include the following:

\section{Introduction (2O pts)}

What are the disease and its significance?

Materials \& Methods (35 points)

What is your specific target (ex. receptor on type of cell) and why is it a good choice of target?

What type of nanoparticle did you select and why?

What are the specifications of your nanoparticles (size, charge, etc.) and your rationale for each choice?

What is your route of administration and how is it ideal for your situation?

\section{Results \& Discussion (35 points)}

Create mock data that shows the concentration versus time. Calculate the clearance, AUC, and half-life.

Diagram the compartment model that you would use and write out the equations. Explain why you chose that type of compartment model.

What is the specificity?

What is the biodistribution? 


\section{Document A2.3: Example debate moderator material}

You have been selected to be a moderator for the upcoming debate: Imaging Costs. In this debate, 2 assigned teams of students will debate whether or not low-dose computed tomography scans should be implemented as part of the Medicare program to screen for lung cancer. The debate will go as follows: Each team will have 20 minutes to prepare their opening statement, principle argument, and closing statement. You will flip a coin to determine which team (pro-imaging or anti-imaging) says their opening statement first- this will be team 1. During the preparatory portion, you will listen in for 10 minutes to team 1 and then 10 minutes to team 2 and prepare any questions that you might want to interject.

During the debate portion, you will adhere to the following schedule (cut off students who continue past the time limit):

Team 1 Opening Statement (3 minutes)

Team 2 Opening Statement (3 minutes)

Team 1 Principle Argument (5 minutes)

Team 2 Rebuttal (2 minutes)

Team 2 Principle Argument (5 minutes)

Team 1 Rebuttal (2 minutes)

Team 1 Closing Statement (3 minutes)

Team 2 Closing Statement (3 minutes)

You reserve the right to interject in between sections to ask clarifying (or probing) questions. You are also responsible for ensuring that teams do not directly contradict their "facts." Each team will only be privy to their own facts, whereas you have access to both sides. 
At the conclusion of the debate, you will decide whether or not to implement the imaging technology. 
APPENDIX 3

\section{List of publications and awards}




\section{Publications}

1. Brinton LT*, Bauknight DK*, Dasa SSK, Kelly KA. (2016) "PHASTpep: analysis software for discovery of cell-selective peptides via phage display and nextgeneration sequencing.” PLoS One. Revision under review.

2. Evans J, Essex A, Xin H, Amitai N, Brinton LT, Griner E. (2015) “Registered report: Wnt activity defines colon cancer stem cells and is regulated by the microenvironment.” Elife. E07301, Available: http://elifesciences.org/content/4/eo7301

3. Dasa SSK, Suzuki R, Gutknecht M, Brinton LT, Tian Y, Michaelsson E, Lindfors L, Klibanov AL, French B, Kelly KA. (2015) “Development of target-specific liposomes for delivering of small molecule drugs after reperfused myocardial infarction.” Journal of Controlled Release. Epub. PMID: 26122651

4. Brinton LT, Curley CT, Kelly K. (2015) “The LAWA technique implemented in a course in nanomedicine." Proceedings for the 2015 American Society for Engineering Education (ASEE) Annual Conference and Exposition. Available: http://www.asee.org/public/conferences/56/papers/12753/view

5. Brinton LT, Sloane HS, Kester M, Kelly KA. (2015) "Formation and role of exosomes in cancer." Cellular and Molecular Life Sciences 72(4):659-71. PMID: 25336151

6. Brinton LT, Brentnall TA, Smith JA, Kelly KA. (2012) "Metastatic biomarker discovery through proteomics." Cancer Genomics and Proteomics 9:345-55. PMID: 23162074

* denotes equal contribution 


\section{Awards}

1. All-University Graduate Teaching Assistant Award (\$1000), Spring 2015

2. UVA Robert J. Huskey Research Exhibition, ${ }^{\text {nd }}$ place (\$200), Spring 2015

3. SEAS Voigt Teaching Internship (tuition, stipend, insurance), Fall 2014

4. ASEE BED Travel Award (\$300), Spring 2014

5. People's Choice Award, International 3-Minute Thesis Competition, Universitas21 (\$300), Fall 2013

6. UVA 3-Minute Thesis Competition, $1^{\text {st }}$ place, Fall 2013

7. NIH Pharmacology Training Grant (tuition, stipend, insurance), Summer 2011 Spring 2013 